TRANSACTIONS OF THE

AMERICAN MATHEMATICAL SOCIETY

Volume 350, Number 10, October 1998, Pages 3837-3901

S $0002-9947(98) 02279-\mathrm{X}$

\title{
A NONLINEAR FOKKER-PLANCK EQUATION MODELLING THE APPROACH TO THERMAL EQUILIBRIUM IN A HOMOGENEOUS PLASMA
}

\author{
M. ESCOBEDO, M. A. HERRERO, AND J. J. L. VELAZQUEZ
}

AbStRaCt. This work deals with the problem consisting in the equation

$$
\frac{\partial f}{\partial t}=\frac{1}{x^{2}} \frac{\partial}{\partial x}\left[x^{4}\left(\frac{\partial f}{\partial x}+f+f^{2}\right)\right], \quad \text { when } \quad x \in(0, \infty), t>0,
$$

together with no-flux conditions at $x=0$ and $x=+\infty$, i.e.

$$
x^{4}\left(\frac{\partial f}{\partial x}+f+f^{2}\right)=0 \quad \text { as } x \longrightarrow 0 \text { or } x \longrightarrow+\infty .
$$

Such a problem arises as a kinetic approximation to describe the evolution of the radiation distribution $f(x, t)$ in a homogeneous plasma when radiation interacts with matter via Compton scattering. We shall prove that there exist solutions of (1), (2) which develop singularities near $x=0$ in a finite time, regardless of how small the initial number of photons $N(0)=\int_{0}^{+\infty} x^{2} f(x, 0) d x$ is. The nature of such singularities is then analyzed in detail. In particular, we show that the flux condition (2) is lost at $x=0$ when the singularity unfolds. The corresponding blow-up pattern is shown to be asymptotically of a shock wave type. In rescaled variables, it consists in an imploding travelling wave solution of the Burgers equation near $x=0$, that matches a suitable diffusive profile away from the shock. Finally, we also show that, on replacing (2) near $x=0$ as determined by the manner of blow-up, such solutions can be continued for all times after the onset of the singularity.

\section{INTRODUCTION}

We are concerned in this work with the study of the equation

$$
\frac{\partial f}{\partial t}=\frac{1}{x^{2}} \frac{\partial}{\partial x}\left[x^{4}\left(\frac{\partial f}{\partial x}+f+f^{2}\right)\right], \quad \text { when } \quad x \in(0, \infty), t>0,
$$

together with the following initial and boundary conditions. At $t=0$ we shall require that

$$
f(x, 0)=F(x), \quad \text { for } \quad 0 \leq x<\infty,
$$

where $F$ is a given continuous, nonnegative and bounded function such that

$$
\int_{0}^{\infty} x^{2} F(x) d x<\infty
$$

Received by the editors October 15, 1996.

1991 Mathematics Subject Classification. Primary 35K55, 35B40.

The first author was partially supported by DGICYT Grant PB93-1203 and EEC Contract ERB 4061 PL 95-0545.

The second and third authors were partially supported by DGICYT Grant PB93-0438 and EEC Contract CHRX-CT-0413. 
Finally, as boundary conditions we will impose the vanishing of the corresponding flux as $x \longrightarrow 0$ and $x \longrightarrow \infty$, i.e.,

$$
x^{4}\left(\frac{\partial f}{\partial x}+f+f^{2}\right) \longrightarrow 0, \quad \text { as } \quad x \longrightarrow 0 \quad \text { or } \quad x \longrightarrow \infty .
$$

Problem (1.1) has been proposed by Kompaneets (cf. [K]) to describe the evolution of the radiation distribution $f(x, t)$ in a homogeneous plasma when radiation interacts with matter via Compton scattering. In that context the space coordinate $x$ represents a momentum coordinate and $t$ denotes time. More precisely, an equation which includes (1.1a) as a particular case (cf. (1.2) below) is obtained in $[\mathrm{K}]$ as a leading term for the corresponding Boltzmann equation under the crucial assumption that the scattering cross section is of the classical Thomson type, as represented by the coefficient $\alpha(x)=x^{4}$ in (1.1a). The complete approximating equation obtained in $[\mathrm{K}]$ reads as follows

$$
\frac{\partial f}{\partial t}=\frac{1}{x^{2}} \frac{\partial}{\partial x}\left[x^{4}\left(\frac{\partial f}{\partial x}+f+f^{2}\right)\right]+\sigma(x)\left(f_{0}-f\right) .
$$

The second term on the right in (1.2a) accounts for the Bremsstrahlung effects. Here $\sigma$ is the emission-absorption rate, which is taken to be of the form

$$
\sigma(x)=\varepsilon x^{-3} e^{-\frac{x}{2}} K_{0}\left(\frac{x}{2}\right),
$$

where $\varepsilon>0$ is a small parameter and $K_{0}$ is the zero order modified Bessel function. As to the term $f_{0}=f_{0}(x)$ in (1.2a), it is obtained by setting $\mu=0$ in the expression for the Bose-Einstein distribution

$$
f_{\mu}(x)=\frac{1}{\left(e^{x+\mu}-1\right)}, \quad \text { where } \quad \mu \geq 0 .
$$

A question which naturally arises is that of determining the asymptotic behaviour of solutions of the problem (1.2), (1.1b), (1.1c) and (1.1d). A first step in this direction was taken by Caflisch and Levermore in [CL], under the assumption that Bremsstrahlung effects are negligible. This accounts for setting $\sigma \equiv 0$ in $(1.2 a)$, thus reducing such an equation to (1.1a). An interesting feature of such a simplified case is that the quantity

$$
N(t) \equiv \int_{0}^{\infty} x^{2} f(x, t) d x
$$

which represents the total number of photons at time $t$, is actually constant in time. In view of $(1.1 \mathrm{c})$, one then has

$$
N(f) \equiv \int_{0}^{\infty} x^{2} f(x, t) d x=\int_{0}^{\infty} x^{2} F(x) d x=N(0)<\infty .
$$

On the other hand, if we define the entropy function

$$
S(t)=\int_{0}^{\infty} x^{4}\left(f+f^{2}\right)^{-1}\left(\frac{\partial f}{\partial x}+f+f^{2}\right)^{2} d x,
$$

we can readily check that it satisfies

$$
\frac{\partial S(t)}{\partial t} \geq 0
$$


along the solutions of (1.1). An additional fact to be taken into account is that

$$
\left\{\begin{array}{l}
\text { For any } \mu \geq 0, f_{\mu} \text { given in } \quad(1.3) \text { is a stationary solution of (1.1a). } \\
\text { Moreover } N\left(f_{\mu}\right) \text { is decreasing as a function of } \mu \text {, and in particular } \\
\text { one has that for all } \mu \geq 0, \quad N\left(f_{\mu}\right) \leq N\left(f_{0}\right)=\int_{0}^{\infty} \frac{x^{2}}{\left(e^{x}-1\right)} d x
\end{array}\right.
$$

Taking into account (1.4), (1.6) and (1.7), a possible scenario for the long-time asymptotics of (1.1) was proposed in [CL] that may be summarized as follows. If $F$ in (1.1) is such that

$$
N(F)=\int_{0}^{\infty} x^{2} F(x) d x \leq N\left(f_{0}\right),
$$

then, asymptotically as $t \longrightarrow \infty$, the corresponding solution of (1.1) approaches the Bose-Einstein distribution in (1.3), whose total number of photons coincides with that of $F$. In other words, the parameter $\mu$ in (1.3) has to be selected so that

$$
N\left(f_{\mu}\right)=N(F)
$$

If on the other hand $N(F)>N\left(f_{0}\right)$, the numerical analysis in [CL] suggests that the equilibrium radiation should asymptotically decouple in the following manner

$$
f(x, t) \sim f_{0}(x)+\left(N(F)-N\left(f_{0}\right)\right) x^{-2} \delta(x), \quad \text { for } \quad 0 \leq x<\infty, t \gg 1,
$$

where $\delta(x)$ denotes Dirac's delta centered at the origin. One should then have asymptotic condensation at the state of zero energy, and the above result would illustrate a way of approaching a Bose-Einstein condensate.

At this juncture, it is worth mentioning that the analysis carried out in [CL] requires that near the origin solutions satisfy an estimate of the form

$$
f(x, t) \leq \frac{c(t)}{x} \quad \text { for } \quad 0<x<1, \quad t>0
$$

for some continuous and nonnegative function $c(t)$. Notice that Bose-Einstein distributions do satisfy (1.9) with $c \equiv 1$. The results in [CL] can then be rephrased as stating that Bose-Einstein condensation would be achieved for those solutions of (1.1) with large photon number for which the bound (1.9) holds.

We prove in this paper that problem (1.1) has solutions for which (1.9) fails to hold after a finite time. As a matter of fact, we shall construct solutions for which not only (1.9), but also the flux condition (1.1d) break down near $x=0$ in finite time. More precisely, the following result is obtained.

Theorem 1. There exist smooth nonnegative functions $F$ for which problem (1.1) has a solution defined in a time interval $(0, T)$, with $0<T<\infty$, and such that (1.1d) no longer holds at $x=0$ when $t=T$.

It is interesting to observe that such solutions can be uniquely continued for all times after such blow-up occurs. To prove such a result, we make use of the following.

Theorem 2. Consider problem $(\mathrm{P})$ consisting of $(1.1 \mathrm{a})-(1.1 \mathrm{c})$ together with the boundary conditions

$$
\begin{aligned}
& \text { There exists } \quad A>0 \quad \text { such that } \\
& f(x, t) \leq \frac{A}{x^{2}}, \quad \text { for } \quad x \in(0,1), \text { and } t>0,
\end{aligned}
$$




$$
\lim _{x \longrightarrow \infty}\left(x^{4}\left(\frac{\partial f}{\partial x}+f+f^{2}\right)\right)=0 .
$$

Then, if $F$ satisfies the decay assumption

$$
\lim _{x \rightarrow \infty} x^{4} F(x)=0,
$$

problem $(\mathrm{P})$ has a unique global solution. Moreover, in such a case,

$$
\lim _{x \longrightarrow \infty} x^{4} f(x, t)=0 \text { for all } t>0
$$

We finally proceed to analyze in detail the manner of blow-up near $x=0$ for a particular class of solutions. Namely, we obtain

Theorem 3. For any given positive constants $C$ and $T$, there exists a function $F$ satisfying (1.1c) for which problem (1.1) has a unique solution for times $0<t<T$, and such that the following properties hold:

$$
\lim _{t \longrightarrow T^{-}}(T-t)^{2} f(z(T-t), t)=\left\{\begin{array}{l}
\frac{C}{z^{2}} \quad \text { if } z>C, \\
0 \quad \text { if } 0<z<C,
\end{array}\right.
$$

where convergence is uniform on compact subsets of $(C,+\infty)$ and $(0, C)$ respectively, and

$$
\lim _{x \rightarrow 0} x^{2} f(x, T)=C^{\prime},
$$

for some positive constant $C^{\prime}$ that depends on $F$.

A point that we want to stress herein is the onset of a discontinuity in (1.13). Actually, an interesting feature of the blow-up mechanism for (1.1) is the development of a Burgers-type shock structure for $f(x, t)$ near $z=C$ with width $O\left((T-t)^{2}\right)$ when measured in the $x$-variable. The detailed structure of the corresponding boundary layer will be described later in the corresponding section.

We wish to point out that our analysis does not preclude the existence of solutions satisfying (1.9). It will be apparent from our approach that we can obtain global solutions for which (1.9) holds if we start from initial values lying below one of the Bose-Einstein distributions given in (1.3). We expect, however, that any solution with large enough initial photon number will blow-up near $x=0$ in finite time, and we intend to address this question elsewhere. In view of the blow-up phenomenon described herein, one may wonder whether such a fact would still occur when (1.1a) is replaced by (1.2a), i.e. Bremsstrahlung effects are retained. The answer to that question is negative. The corresponding argument, however, involves a rather technical boundary layer analysis, and will be explained elsewhere.

We conclude this Introduction by describing the plan of this paper. To begin with, we prove Theorem 1 in Section 2 below. We then prove Theorem 2 in Section 3. Having done that, we proceed with the proof of Theorem 3. In view of the technicalities which are involved, we have thought it convenient to explain first the formal arguments leading to the construction of such solutions by means of matched asymptotic expansions. This is done in Section 4. A rigorous proof of Theorem 3 is then presented in the final Section 5 . 


\section{The Proof of Theorem 1: Existence}

We begin this section by proving local existence of solutions to the problem (1.1a) - (1.1d) for some initial data. We then show that condition (1.9) may fail to hold after a finite time, even for distributions corresponding to an arbitrarily small initial photon number. A detailed analysis of blow-up patterns for a particular class of solutions will be postponed to Sections 4 and 5 .

Here and henceforth, we shall find it convenient to work with different versions of (1.1) obtained under suitable changes of variables. For instance, if we set

$$
x=e^{y}, \quad x^{2} f(x, t)=e^{y} v(y, t),
$$

a quick check reveals that (1.1a) is transformed into

$$
v_{t}=v_{y y}+v_{y}-2 v+2 v^{2}+2 v v_{y}+e^{y}\left(3 v+v_{y}\right), \quad \text { for } \quad y \in(-\infty,+\infty), t>0 .
$$

We then readily see that $(2.2)$ has supersolutions $\bar{v}(y, t)$ of the form

$$
\bar{v}(y, t ; a, b) \equiv \bar{v}(y, t)=\left\{\begin{array}{l}
C_{1} e^{a t-b y}, \quad \text { for } y \geq y_{0}, t>0, \\
C_{2} e^{a t-y}, \quad \text { for } y<y_{0}, t>0 .
\end{array}\right.
$$

where $C_{1}$ is an arbitrary positive constant and $y_{0}, a, b, C_{2}$ are constants satisfying

$$
b>3, \quad a \geq \max \left(b^{2}-b-2,2 e^{y_{0}}-2\right), \quad \text { and } \quad C_{1} e^{-b y_{0}}=C_{2} e^{-y_{0}} .
$$

Our starting point is the following.

Lemma 2.1. For every initial data $F$ such that $x F(x)$ is bounded and satisfies

$$
0 \leq x F(x) \leq \bar{v}(\log x, 0), \quad \text { for } x \in \mathbf{R},
$$

for some supersolution $\bar{v}$, there is a classical global solution $f$ of (1.1a) such that

(1) For all $t \geq 0$,

$$
x f(x, t) \leq \begin{cases}C_{1} e^{a t} x^{-b} & \text { if } \log x \geq y_{0}, \\ C_{2} e^{a t} x^{-1} & \text { if } \log x \leq y_{0} .\end{cases}
$$

(2) There exist $t_{0}>0$ and a positive constant $C$ such that

$$
x f(x, t) \leq C \quad \text { for all } x \in(0,+\infty), t \in\left[0, t_{0}\right] .
$$

(3) For all $t \in\left(0, t_{0}\right)$,

$$
\lim _{x \longrightarrow+\infty} x^{4}\left(f(x)+f^{2}(x)+f_{x}(x)\right)=0, \quad \lim _{x \longrightarrow 0} x^{4}\left(f(x)+f^{2}(x)+f_{x}(x)\right)=0 .
$$

Proof. By using the change of variables (2.1) we are led to consider equation (2.2) with bounded initial condition $v_{0}(y)=e^{y} F\left(e^{y}\right) \in L^{\infty}(\mathbf{R})$. In order to prove existence of a solution we first replace equation (2.2) by

$$
v_{n t}=v_{n y y}+v_{n y}-2 v_{n}+2 \psi_{n}\left(v_{n}\right)+2 v_{n} v_{n y}+\varphi_{n}(y)\left(3 v_{n}+v_{n y}\right) .
$$

where the functions $\psi_{n}$ and $\varphi_{n}$ are smooth and nonnegative, and such that

$$
\varphi_{n}(z)= \begin{cases}e^{z} & \text { if }|z|<n \\ e^{n+1} & \text { if }|z|>n+1\end{cases}
$$


and

$$
\psi_{n}(z)=\left\{\begin{array}{l}
z^{2} \quad \text { if }|z|<n \\
(n+1)^{2} \quad \text { if }|z|>n+1
\end{array}\right.
$$

A quick check reveals that the functions $\bar{v}$ given in (2.3) are supersolutions of (2.6) for every $n \geq 1$. By classical results, we now have that for any bounded initial data $v_{0}$, equation (2.6) has a unique local classical solution $v_{n} \in C\left(\left(0, T_{n}\right) ; L^{\infty}(\mathbf{R})\right.$. Moreover, by hypothesis $v_{0} \leq \bar{v}(\cdot, 0)$ for some supersolution given in $(2.3)$. Therefore, by the maximum principle we deduce that $v_{n} \leq \bar{v}$. This shows that the sequence $\left\{v_{n}\right\}$ is uniformly bounded in $L_{\text {loc }}^{\infty}\left(\mathbf{R} \times \mathbf{R}^{+}\right)$. By standard parabolic regularity, the sequence is then bounded in $C^{2,1}\left(\mathbf{R} \times \mathbf{R}^{+}\right)$. Therefore there exist a subsequence, still denoted by $\left\{v_{n}\right\}$, and a function $v \in C^{2,1}\left(\mathbf{R} \times \mathbf{R}^{+}\right)$such that $\left\{v_{n}\right\}$ converges to $v$ uniformly on every compact subset of $\mathbf{R} \times \mathbf{R}^{+}$. Moreover, $v$ is a classical solution of (2.2) and satisfies $v \leq \bar{v}$ on $\mathbf{R} \times \mathbf{R}^{+}$. This shows (1) of Lemma 2.1.

Using the integral equation asociated to (2.6) it is easy to show that, provided $v_{n}$ exists,

$$
\begin{aligned}
\left\|v_{n}(t)\right\|_{\infty} \leq & \left\|v_{0}\right\|_{\infty}+\int_{0}^{t}\left((t-s)^{-1 / 2}+2\right)\left\|v_{n}(s)\right\|_{\infty} d s \\
& +\int_{0}^{t}\left((t-s)^{-1 / 2}+2\right)\left\|v_{n}(s)\right\|_{\infty}^{2} d s+\int_{0}^{t}\left((t-s)^{-1 / 2}+4\right)\left\|e^{y} v_{n}(s)\right\|_{\infty} d s .
\end{aligned}
$$

Since

$$
e^{y} v_{n}(y, s) \leq\left\{\begin{array}{l}
e^{y_{0}} v_{n} \quad \text { if } y<y_{0} \\
C_{1} e^{a t-(b-1) y} \quad \text { if } y>y_{0}
\end{array}\right.
$$

we deduce that

$$
\begin{aligned}
\left\|v_{n}(t)\right\|_{\infty} \leq & \left\|v_{0}\right\|_{\infty}+\int_{0}^{t}\left((t-s)^{-1 / 2}+2\right)\left\|v_{n}(s)\right\|_{\infty} d s \\
& +\int_{0}^{t}\left((t-s)^{-1 / 2}+2\right)\left\|v_{n}(s)\right\|_{\infty}^{2} d s \\
& +e^{y_{0}} \int_{0}^{t}\left((t-s)^{-1 / 2}+4\right)\left\|v_{n}(s)\right\|_{\infty} d s+C_{1} \int_{0}^{t}\left((t-s)^{-1 / 2}+4\right) e^{a s} d s .
\end{aligned}
$$

Observe now that for every value of $\left\|v_{0}\right\|_{\infty}$ there is a solution $\rho(t)$ of the equation

$$
\begin{aligned}
\rho(t)= & \left\|v_{0}\right\|_{\infty}+\int_{0}^{t}\left((t-s)^{-1 / 2}+2\right) \rho(s) d s+\int_{0}^{t}\left((t-s)^{-1 / 2}+2\right) \rho^{2}(s) d s \\
& +e^{y_{0}} \int_{0}^{t}\left((t-s)^{-1 / 2}+4\right) \rho(s) d s+C_{1} \int_{0}^{t}\left((t-s)^{-1 / 2}+4\right) e^{a s} d s
\end{aligned}
$$


defined for $0 \leq t \leq t_{0}$, for some positive and finite $t_{0}$ depending on $\left\|v_{0}\right\|_{\infty}$. Moreover,

$$
\begin{aligned}
\left(\left\|v_{n}(t)\right\|_{\infty}-\rho(t)\right)^{+} \leq & \int_{0}^{t}\left((t-s)^{-1 / 2}+2\right)\left(\left\|v_{n}(s)\right\|_{\infty}-\rho(s)\right)^{+} d s \\
& +\int_{0}^{t}\left((t-s)^{-1 / 2}+2\right)\left(\left\|v_{n}(s)\right\|_{\infty}^{2}-\rho^{2}(s)\right)^{+} d s \\
& +e^{y_{0}} \int_{0}^{t}\left((t-s)^{-1 / 2}+4\right)\left(\left\|v_{n}(s)\right\|_{\infty}-\rho(s)\right)^{+} d s \\
\leq & \int_{0}^{t}\left((t-s)^{-1 / 2}+2\right)\left(\left\|v_{n}(s)\right\|_{\infty}-\rho(s)\right)^{+} d s \\
& +\gamma_{n} \int_{0}^{t}\left((t-s)^{-1 / 2}+2\right)\left(\left\|v_{n}(s)\right\|_{\infty}-\rho(s)\right)^{+} d s \\
& +e^{y_{0}} \int_{0}^{t}\left((t-s)^{-1 / 2}+4\right)\left(\left\|v_{n}(s)\right\|_{\infty}-\rho(s)\right)^{+} d s,
\end{aligned}
$$

where $\gamma_{n}=\max \left(\left\|v_{n}\right\|_{L^{\infty}\left(\mathbf{R}^{+} \times \mathbf{R}\right)},\|\rho\|_{L^{\infty}\left(0, t_{0} / 2\right)}\right)$. By Gronwall's inequality we deduce that $v_{n}(y, t) \leq \rho(t)$ for all $y$ in $\mathbf{R}$ and for every $t \in\left[0, t_{0} / 2\right]$, and by passing to the limit, so is $v$. This shows (2) in Lemma 2.1.

On the other hand, since $v$ is uniformly bounded on $\mathbf{R} \times\left[0, t_{0}\right]$, we deduce that

$$
\lim _{y \longrightarrow-\infty}\left(e^{3 y} v(y, t)+e^{2 y} v(y, t)\right)=0
$$

uniformly for $t \in\left[0, t_{0}\right]$, whence

$$
\lim _{x \longrightarrow 0} x^{4}\left(f(x, t)+f^{2}(x, t)\right)=0 .
$$

Using the boundedness of $v$ and standard regularity results, we find that the solutions of (2.2) satisfy

$$
\left|v_{y}\right| \leq C_{\delta} \quad \text { for } \delta<t<t_{0}
$$

whence

$$
\left|f_{x}\right| \leq \frac{C}{x^{2}} \quad \text { for } \delta<t<t_{0} .
$$

Moreover, observe that, by the form of the supersolution $\bar{v}$ given in (2.3a),

$$
f(x, t) \leq \frac{C_{2} e^{a t}}{x^{b+1}} \quad \text { for } \quad x \gg 1,
$$

so that, since $b>3$,

$$
\lim _{x \longrightarrow \infty} x^{4}\left(f+f^{2}\right)=0 .
$$

To control the term $f_{x}$ in (1.1d) we argue as follows. For any $R>1$, we define

$$
g_{R}(x, t)=R^{4} f(x+R, t)
$$

so that $g_{R}$ satisfies the equation

$$
\frac{\partial g}{\partial t}=\frac{1}{(x+R)^{2}} \frac{\partial}{\partial x}\left[(x+R)^{4}\left(\frac{\partial g}{\partial x}+g+\frac{g^{2}}{R^{4}}\right)\right] .
$$

By (2.10), we have that $g_{R}(x, t) \longrightarrow 0$ as $R \longrightarrow \infty$ uniformly on sets where $|x| \leq 1$ and $0 \leq t \leq T$. On the other hand, in such regions, (2.11) can be viewed as a diffusion equation having a diffusion coefficient $D(x, R)=O\left(R^{2}\right)$ for $R \gg 1$. If 
we assume for a moment that $D(x, R) \equiv R^{2}$, then we could absorb this coefficient by defining a new time scale $s=R^{2} t$. We would then have that $g_{R}(x, s) \longrightarrow 0$ as $R \longrightarrow \infty$, uniformly on sets $|x| \leq 1$ and $0 \leq s \leq R^{2} T$. Classical regularity theory then yields

$$
\left|\frac{\partial g_{R}}{\partial x}\right| \longrightarrow 0, \quad \text { as } \quad R \longrightarrow \infty
$$

uniformly on sets $|x| \leq 1 / 2$ and $1 \leq s \leq R^{2} T$. Hence

$$
\left|\frac{\partial f}{\partial x}\right|=o\left(\frac{1}{x^{4}}\right) \quad \text { as } \quad x \longrightarrow \infty
$$

uniformly on sets $\frac{1}{x^{2}} \leq t \leq T$. Putting together (2.11) and (2.13), we see that (1.1d) holds as $x \longrightarrow \infty$ under our current assumptions. The case $D(x, R)=O\left(R^{2}\right)$ for large $R$ follows by recalling (cf. [A]) that fundamental solutions of the heat equation provide upper and lower bounds for fundamental solutions of parabolic equations of the type

$$
u_{t}=\sum_{i, j}\left(a_{i, j}(x, t) u_{x_{i}}\right)_{j}
$$

provided that

$$
\lambda|\xi|^{2} \leq \sum a_{i, j}(x, t) \xi_{i} \xi_{j} \leq \Lambda|\xi|^{2},
$$

for some positive constants $\Lambda>\lambda>0$ and any vector $\xi=\left(\xi_{1}, \cdots, \xi_{N}\right)$. From this we obtain (1.1d) when $x \longrightarrow \infty$ via standard representation formulas for the solutions of (2.12).

We next prove the following singularity result for solutions of $(1.1 \mathrm{a})-(1.1 \mathrm{~d})$.

Lemma 2.2. For every $\varepsilon>0$, there exists initial data $F_{\varepsilon}$ satisfying $(2.4)$ such that the corresponding solution $f_{\varepsilon}$ of (1.1) given by Lemma 2.1 satisfies

$$
\int_{0}^{\infty} x^{2} f_{\varepsilon}(x, t) d x=\varepsilon \text { for all } t>0
$$

whereas, if we set

$$
G_{\beta}(t)=\int_{0}^{1} x^{\beta} f_{\varepsilon}^{2}(x, t) d x
$$

then

$$
\begin{aligned}
& \text { For any } \beta \text { such that } 2<\beta<3 \text { there exists } t_{*}=t_{*}\left(\beta, F_{\varepsilon}\right)<\infty \text { at which } \\
& G_{\beta}(t) \text { becomes unbounded. }
\end{aligned}
$$

Notice that this result implies that, regardless of how small the initial number of photons is, we may find radiation distributions for which (1.9) ceases to hold in a finite time. Notice also that (2.15) is compatible with the bound (1.10a) required for global existence in Theorem 2.

Proof. Let $f(x, t)$ be any of the solutions obtained in Lemma 2.1. Clearly one has

$$
\begin{aligned}
N(f) \equiv \int_{0}^{\infty} x^{2} f(x, t) d x & =\int_{-\infty}^{\infty} v(y, t) e^{2 y} d y \\
& \leq \int_{0}^{\infty} \bar{v}(y, t) e^{2 y} d y<\infty .
\end{aligned}
$$


In view of our choice of supersolution in (2.3), we also have

$$
\int_{0}^{\infty} x^{\beta+1} f^{2}(x, t) d x=\int_{-\infty}^{\infty} v^{2}(y, t) e^{\beta y} d y<\infty,
$$

provided that $2<\beta<2 b$. We now claim that the solutions just obtained are such that the function

$$
G_{\beta}(t)=\int_{0}^{\infty} x^{\beta} f^{2}(x, t) d x \equiv \int_{-\infty}^{+\infty} e^{(\beta-1) y} v^{2}(y, t) d y
$$

cannot exist for all $t>0$ if $2<\beta<3$. In order to show this we argue as follows. Set $\alpha=\beta-1=k+r$, where $0<k<\min (1, r)$, and define

$$
\Phi_{r}(y)=\lambda_{r} \exp \left(-r \sqrt{1+y^{2}}\right),
$$

where $\lambda_{r}>0$ is selected so that

$$
\int_{-\infty}^{+\infty} \Phi_{r}(y) e^{k y} d y=1
$$

We next consider a smooth, nonnegative cut-off function $\phi$ such that $0 \leq \phi \leq 1$, $\phi(x)=1$ if $|x| \leq 1, \phi(x)=0$ if $|x| \geq 3$ and $\left|\phi^{\prime}\right| \leq 1$. We then define a sequence $\phi_{n}$ through the relation $\phi_{n}(x)=\phi(x / n)$ for $n=1,2, \cdots$. To proceed further we rewrite (2.2) in the form

$$
v_{t}=\left(e^{k y} v_{y}\right)_{y} e^{-k y}+(1-k) v_{y}-2 v+2 v^{2}+\left(v^{2}\right)_{y}+e^{y}\left(3 v+v_{y}\right) .
$$

We now multiply both sides of (2.20) by $e^{k y} \phi_{n}(y) \Phi_{r}(y)$ and integrate over the whole line. Dropping the subscript $n$ for convenience, a routine computation gives us

$$
\begin{aligned}
& \frac{d}{d t} \int_{\mathbf{R}} v \Phi_{r} \phi e^{k y} d y=\int_{\mathbf{R}} v\left(e^{k y}(\Phi \phi)_{y}\right)_{y} d y+(k-1) \int_{\mathbf{R}} v \phi_{y} \Phi_{r} e^{k y} d y \\
& +(k-1) \int_{\mathbf{R}} v \phi \Phi_{r y} e^{k y} d y+[k(k-1)-2] \int_{\mathbf{R}} v \Phi_{r} \phi e^{k y} d y \\
& +(2-k) \int_{\mathbf{R}} v^{2} \Phi_{r} \phi e^{k y} d y-\int_{\mathbf{R}} v^{2} \Phi_{r} \phi_{y} e^{k y} d y-\int_{\mathbf{R}} v^{2} \Phi_{r y} \phi e^{k y} d y \\
& +[3-k-1] \int_{\mathbf{R}} v \Phi_{r} \phi e^{(k+1) y} d y-\int_{\mathbf{R}} v \Phi_{r} \phi_{y} e^{(k+1) y} d y-\int_{\mathbf{R}} v \phi \Phi_{r y} e^{(k+1) y} d y .
\end{aligned}
$$

On the other hand, one readily sees that

$$
\Phi_{r}^{\prime \prime} \geq-r \Phi_{r}, \quad \Phi_{r}^{\prime \prime}+k \Phi_{r}^{\prime} \geq-r(k+1) \Phi_{r} .
$$

Taking this into account, we deduce that

$$
\begin{gathered}
\int_{\mathbf{R}} v\left(e^{k y}\left(\Phi_{r} \phi\right) y\right)_{y} d y \geq \int_{\mathbf{R}}\left(\phi_{y y} \Phi_{r}+2 \Phi_{r y} \phi_{y}+k \phi_{y} \Phi_{r}\right) v e^{k y} d y \\
-r(k+1) \int_{\mathbf{R}} v \Phi_{r} \phi e^{k y} d y
\end{gathered}
$$

and

$$
(2-k) \int_{\mathbf{R}} v^{2} \Phi_{r} \phi e^{k y} d y-\int_{\mathbf{R}} v^{2} \Phi_{r y} \phi e^{k y} d y \geq(2-k-r)\left(\int_{\mathbf{R}} v \Phi_{r} \phi e^{k y} d y\right)^{2},
$$


where we have also made use of (2.19) to derive the last inequality above. Plugging (2.23) into (2.21), we derive

$$
\begin{aligned}
\frac{d}{d t} \int_{\mathbf{R}} v \Phi_{r} \phi e^{k y} d y \geq & c(r, k) \int_{\mathbf{R}} v \Phi_{r} \phi e^{k y} d y+(2-(k+r))\left(\int_{\mathbf{R}} v \Phi_{r} \phi e^{k y} d y\right)^{2} \\
& +(2-(k+r)) \int_{\mathbf{R}} v \Phi_{r} \phi e^{(k+1) y} d y+\Psi_{n}(t),
\end{aligned}
$$

where $c(r, k)=k^{2}-k(1+2 r)-2$, and

$$
\begin{aligned}
\Psi_{n}(t)= & \int_{\mathbf{R}}\left(\phi_{y y} \Phi_{r}+2 \phi_{y} \Phi_{r y}+k \phi_{y} \Phi_{r}\right) e^{k y} d y+(k-1) \int_{\mathbf{R}} v \Phi_{r} \phi_{y} e^{k y} d y \\
& -\int_{\mathbf{R}} v^{2} \Phi_{r} \phi_{y} e^{k y} d y-\int_{\mathbf{R}} v \Phi_{r} \phi_{y} e^{(k+1) y} d y .
\end{aligned}
$$

Notice that we have kept the subscript $n$ in the left of (2.24b) to stress the dependence on $n$. As a matter of fact, we use this dependence to observe that

$$
\begin{aligned}
& \text { If } \int_{\mathbf{R}} v^{2} \Phi_{r} e^{k y} d y \quad \text { is finite for any } t>0 \text {, then } \\
& \text { for all } t>0, \quad \lim _{n \longrightarrow \infty} \Psi_{n}(t)=0 .
\end{aligned}
$$

Statement $(2.25)$ follows at once by letting $n \longrightarrow \infty$ in $(2.24 \mathrm{~b})$ and using the dominated convergence theorem. On the other hand, we also have

$$
\lim _{n \longrightarrow \infty}\left(\frac{\partial}{\partial t} \int_{\mathbf{R}} v \Phi_{r} \phi_{n} e^{k y} d y\right)=\frac{\partial}{\partial t} \int_{\mathbf{R}} v \Phi_{r} e^{k y} d y .
$$

It then follows that, letting $n \longrightarrow \infty$ in (2.24),

$$
\frac{\partial}{\partial t} \int_{\mathbf{R}} v \Phi_{r} e^{k y} d y \geq c(r, k) \int_{\mathbf{R}} v \Phi_{r} e^{k y} d y+(2-k-r)\left(\int_{\mathbf{R}} v \Phi_{r} e^{k y} d y\right)^{2},
$$

provided that the assumption in (2.25) holds. Analysis of the differential inequality (2.25) readily shows that $I(t)=\int_{\mathbf{R}} v \Phi_{r} e^{k y} d y$ would blow-up in finite time if $I(0)=$ $\int_{\mathbf{R}} v_{0} \Phi_{r} e^{k y} d y$ is selected large enough, which contradicts (2.25). We have thus shown that the quantity $\int_{\mathbf{R}} v^{2} \Phi_{r} e^{k y} d y$ cannot be global in time. To be more precise, the result actually obtained is the following:

$$
\text { The function } \quad J(t)=\int_{-\infty}^{0} v^{2} e^{(\beta-1) y} d y \quad \text { cannot be global in time. }
$$

To derive (2.28) we merely observe that $e^{-r \sqrt{1+y^{2}}+k y} \sim e^{(r+k) y}=e^{\alpha y}$ for $-y \gg 1$ and $e^{-r \sqrt{1+y^{2}}+k y} \sim e^{-(r-k) y}$ for $y \gg 1$, whence

$$
\begin{aligned}
\int_{0}^{\infty} v^{2} \Phi_{r} e^{k y} d y & \leq C_{1} \int_{0}^{\infty} v^{2} e^{-(r-k) y} d y \\
& \leq C_{2} \int_{0}^{\infty} e^{-2 b y^{2}-(r-k) y} d y<\infty
\end{aligned}
$$

for some positive constants $C_{1}$ and $C_{2}$.

We conclude the proof by observing that the quantity $G_{\beta}(t)$ may blow-up in finite time even if the initial number of photons is arbitrarily small. To wit, we 
take $\gamma \in\left(\frac{\beta-1}{2}, 2\right)$ and define

$$
v_{0}(y)= \begin{cases}e^{-\gamma y} & \text { if }-A<y<-B<0, \\ 0 & \text { otherwise }\end{cases}
$$

and proceed to compare the quantities

$$
I_{1}(t)=\int_{\mathbf{R}} v_{0}^{2} \Phi_{r} e^{k y} d y \quad \text { and } \quad I_{2}(t)=\int_{\mathbf{R}} v_{0} e^{2 y} d y .
$$

A straightforward computation shows that

$$
I_{2}(t)=\int_{-A}^{-B} e^{(2-\gamma) y} d y \leq \int_{-\infty}^{-B} e^{(2-\gamma) y} d y=\frac{e^{-(2-\gamma) B}}{2-\gamma} .
$$

Now, if $B$ is large enough, $\lambda_{r} e^{-r \sqrt{1+y^{2}}} \geq e^{r y} / 2$ provided that $y<-B$. We therefore have

$$
\begin{aligned}
I_{1} & =\lambda_{r} \int_{-A}^{-B} v_{0}^{2} e^{k y} e^{-r \sqrt{1+y^{2}}} d y \\
& \geq \frac{1}{2} \int_{-A}^{-B} e^{-2 \gamma y+\alpha y} d y=\frac{e^{(2 \gamma-\alpha) A}}{2(2 \gamma-\alpha)}\left(1-e^{-(2 \gamma-\alpha)(A-B)}\right) .
\end{aligned}
$$

Therefore, if we select $A-B>\delta>0$, we eventually obtain

$$
\lim _{A \longrightarrow \infty} I_{1}=\infty, \quad \lim _{B \longrightarrow \infty} I_{2}=0,
$$

whereupon the result follows.

\section{A global existence Result. Uniqueness}

In this section we give the proof of Theorem 2 and of the uniqueness statement in Theorem 1. We begin by proving the existence of global solutions for the problem consisting of (1.1a) - (1.1c) and (1.10). It is now convenient to use the auxiliary variable

$$
u(x, t)=x^{2} f(x, t)
$$

A quick check shows that (1.1a) - (1.1c) is then rewritten in the form

$$
\begin{gathered}
u_{t}=\left(x^{2} u_{x}-2 x u+x^{2} u+u^{2}\right)_{x}, \quad \text { for } \quad x \in(0,+\infty), t>0, \\
u(x, 0)=x^{2} F(x), \quad \text { for } \quad x \in(0,+\infty), \\
\left(x^{2} u_{x}-2 x u+x^{2} u+u^{2}\right) \longrightarrow 0 \quad \text { when } x \longrightarrow 0 \text { and } x \longrightarrow \infty .
\end{gathered}
$$

Together with (3.1) we shall also consider the following problem

$$
\begin{gathered}
u_{t}=\left(\varphi_{n}\left(x^{2}\right) u_{x}-2 x u+x^{2} u+u^{2}\right)_{x}, \quad \text { for } x \in(0,+\infty), t>0, \\
u(x, 0)=x^{2} F(x), \text { for } x \in(0,+\infty), \\
u(0, t)=0, \text { for } t>0,
\end{gathered}
$$


where, for $n=1,2, \cdots, \varphi_{n}$ is a $C^{1}$ function such that $\varphi_{n}^{\prime} \geq 0$ and

$$
\varphi_{n}(z)=\left\{\begin{array}{l}
\frac{1}{2 n} \quad \text { if } \quad z \leq \frac{1}{2 n}, \\
z \text { if } \frac{1}{n} \leq z \leq n, \\
2 n \quad \text { if } z \geq 2 n .
\end{array}\right.
$$

By standard results, for every $n$ there is a unique global, classical solution $u_{n}$ to problem (3.2). We now derive suitable bounds on the sequence $u_{n}$. To this end, let us consider the following two auxiliary equations

$$
\begin{gathered}
x^{4}\left(f^{\prime}+f+f^{2}\right)=\alpha, \quad x \in(0, \infty), \\
x^{2} \varphi_{n}\left(x^{2}\right) f^{\prime}+2 x\left(\varphi_{n}\left(x^{2}\right)-x^{2}\right) f+x^{4} f+x^{4} f^{2}=\alpha, \quad x \in(0, \infty),
\end{gathered}
$$

where $\alpha$ is a positive parameter. For $n$ large enough, it is easy to construct supersolutions $\bar{f}_{\alpha}$ of (3.3) and (3.4) of the form

$$
\bar{f}_{\alpha}(x)=\left\{\begin{array}{llr}
\frac{\beta}{x^{2}}, & \text { if } & 0<x<1, \\
\frac{\beta}{x^{4}}, & \text { if } & x>1,
\end{array}\right.
$$

with $\beta$ sufficiently large. Therefore, for any initial data $F$ satisfying the hypothesis of Theorem 2 there exists one of these supersolutions $\bar{f}_{\alpha}$ such that

$$
F(x)<\bar{f}_{\alpha}(x), \text { for all } x>0 \text {, provided that } \beta \text { is large enough. }
$$

Moreover, we claim that if we define

$$
\bar{u}_{n}(x)=x^{2} \bar{f}_{\alpha}(x)
$$

we then have

$$
u_{n}(x, t) \leq \bar{u}_{n}(x), \quad \text { for all } x>0 \text { and } t>0 .
$$

Indeed, by (3.6), (3.8) clearly holds at $t=0$, as well as at $x=0$ for any $t>0$. Since $\bar{u}_{n}$ given by (3.7) is a stationary supersolution of (3.2a), (3.8) follows from the maximum principle. We now deduce from (3.8) that the sequence $\left\{u_{n}\right\}_{n \in \mathbf{N}}$ is bounded in $L^{\infty}\left(\mathbf{R} \times \mathbf{R}^{+}\right)$. By classical parabolic regularity, we then have that $\left\{u_{n}\right\}_{n \in \mathbf{N}}$ is actually bounded in $C^{2,1}\left(\mathbf{R} \times \mathbf{R}^{+}\right)$. Therefore there exist a subsequence, still denoted by $\left\{u_{n}\right\}_{n \in \mathbf{N}}$, and a function $u \in C^{2,1}\left(\mathbf{R} \times \mathbf{R}^{+}\right)$, such that $\left\{u_{n}\right\}_{n \in \mathbf{N}}$ converges to $u$ uniformly on every compact subset of $\mathbf{R} \times \mathbf{R}^{+}$. Moreover, the function $u$ is a classical solution of (3.1a) - (3.1b) and is such that

$$
u(x, t) \leq x^{2} \bar{f}_{\alpha}(x), \quad \text { for all } x>0 \text { and } t>0 .
$$

In particular, by (3.5), we have that $f(x, t)=x^{-2} u(x, t)$, satisfies

$$
f(x, t) \leq \frac{C_{1}}{x^{2}}, \quad \text { for } \quad 0 \leq x \leq 1,
$$

and

$$
f(x, t) \leq \frac{C_{2}}{x^{4}}, \quad \text { for } \quad x \geq 1,
$$

for some given constants $C_{1}$ and $C_{2}$. In order to see that the function $f(x, t)$ defined above is a solution of the problem $(\mathrm{P})$, we need yet to show that condition (1.1d) 
is satisfied as $x \longrightarrow \infty$. To this end, given $R>0$, we consider the function $h(x, t)$ that is a solution to

$$
\left\{\begin{array}{l}
\frac{\partial h}{\partial t}=\frac{1}{x^{2}} \frac{\partial}{\partial x}\left[x^{4}\left(\frac{\partial h}{\partial x}+h+h^{2}\right)\right], \quad \text { when } \quad x \in(R, \infty), t>0, \\
h(x, 0)=\frac{\sigma}{x^{4}}, \quad \text { for } \quad x \geq R, \quad h(R, t)=\frac{C_{2}}{x^{4}}, \quad \text { for } \quad t>0
\end{array}\right.
$$

where $\sigma \in\left(0, C_{2} / 2\right)$ is a constant. Observe first of all that the existence of such a function $h$ follows by a slight modification of the existence proof for the function $f=x^{-2} u$ given above. On the other hand, since by (1.11) $x^{4} F(x) \longrightarrow 0$ as $x \longrightarrow \infty$, we deduce that for any given $\sigma \in\left(0, C_{2} / 2\right), f(x, 0) \equiv F(x) \leq h(x, 0)$ for every $x>R$ if $R$ is large enough. Moreover, by $(3.11), f(R, t) \leq h(R, t)$ for every $t>0$. By the maximum principle we deduce that, for $R$ sufficiently large, $f(x, t) \leq h(x, t)$ for every $x>R$ and $t>0$.

Now set

$$
w(x, t)=x^{4} h(x, t)
$$

Then $w$ satisfies

$$
\begin{gathered}
\frac{\partial \omega}{\partial t}=x^{2} \frac{\partial}{\partial x}\left(w_{x}-\frac{4 w}{x}+w+\frac{w^{2}}{x^{4}}\right), \text { for } x>R, t>0, \\
w(x, 0)=\sigma, \text { for } \quad x>R, \\
w(R, t)=C_{2}, \text { for } t>0 .
\end{gathered}
$$

We next give an estimate of the behaviour of $w$ as $x \longrightarrow \infty$, uniformly in $t$. For this purpose we construct a supersolution of (3.13) which will be larger than $w$ for $R$ large enough. To this end we consider the solution $\omega$ of the following problem

$$
\begin{gathered}
\omega^{\prime}+\left(1-\frac{4}{x}\right) \omega=2 \sigma \quad \text { for } \quad x \geq R, \\
\omega(R)=C_{2},
\end{gathered}
$$

which is explicitly given by

$$
\omega(x)=C_{2} e^{-(x-R)}\left(\frac{x}{R}\right)^{4}+2 \sigma x^{4} e^{-x} \int_{R}^{x} s^{-4} e^{s} d s .
$$

This function is such that $\lim _{x \longrightarrow \infty} \omega(x)=2 \sigma$. If we now set $\bar{\omega}(x)=2 \sigma+\frac{2 \omega}{x}$, then a simple calculation shows that

$$
\bar{\omega}^{\prime}+\left(1-\frac{4}{x}\right) \bar{\omega}<2 \sigma, \quad \text { for all } x \geq R .
$$

Since $\bar{\omega}(R)=2 \sigma+2 C_{2} / R<C_{2} \equiv \omega(R)$ if $R$ is large enough, we deduce by comparison that

$$
\omega(x) \geq 2 \sigma+\frac{2 \omega}{x}, \quad \text { for all } x \geq R .
$$

But this implies that $\omega$ is a supersolution of (3.13), since

$$
\begin{aligned}
x^{2} \frac{\partial}{\partial x}\left(\omega_{x}-\frac{4 \omega}{x}+\omega+\frac{\omega^{2}}{x^{4}}\right) & =x^{2} \frac{\partial}{\partial x}\left(2 \sigma+\frac{\omega^{2}}{x^{4}}\right)=x^{2}\left(\frac{2 \omega \omega^{\prime}}{x^{4}}-\frac{4 \omega^{2}}{x^{5}}\right) \\
& =x^{2} \frac{2 \omega}{x^{4}}\left(2 \sigma-\omega+\frac{2 \omega}{x}\right)<0 .
\end{aligned}
$$


Using (3.13b) and (3.13c) we deduce that, if $R$ is large enough,

$$
w(x, t) \leq \omega(x), \quad \text { for all } x \geq R \text { and } t>0 .
$$

Now, from (3.15), we have

$$
x^{4} f(x, t) \leq w(x, t) \leq C_{2} e^{-(x-R)}\left(\frac{x}{R}\right)^{4}+2 \sigma e^{-x} x^{4} \int_{R}^{x} s^{-4} e^{s} d s .
$$

Next, from (3.15) we also have

$$
x^{4} f(x, t) \leq C_{2} e^{-(x-R)}\left(\frac{x}{R}\right)^{4}+2 \sigma e^{-x} x^{4} \int_{R}^{x} s^{-4} e^{s} d s ;
$$

hence

$$
\lim _{x \rightarrow \infty} x^{4} f(x, t)=2 \sigma
$$

This implies in fact that

$$
\lim _{x \longrightarrow \infty} x^{4} f(x, t)=0,
$$

uniformly in $t>0$. The same argument as in Section 2 proves then that we also have

$$
\lim _{x \longrightarrow \infty} x^{4} \frac{\partial f}{\partial x}=0
$$

uniformly on sets $t \geq \frac{1}{x^{2}}$. This shows that $f$ satisfies (1.1d) as $x \longrightarrow \infty$ and concludes the proof of the existence part in Theorem 2.

Let us prove uniqueness now. To this end we argue by contradiction, assuming that there exist two solutions of problem $(\mathrm{P}), f_{1}$ and $f_{2}$, such that $f_{1}(x, 0)=$ $f_{2}(x, 0)=F(x)$. We then set $g=f_{1}-f_{2}$, so that $g$ satisfies

$$
x^{2} \frac{\partial g}{\partial t}=\frac{\partial}{\partial x}\left(x^{4}\left(g_{x}+g+g\left(f_{1}+f_{2}\right)\right)\right) \quad \text { for } x>0 \text { and } t>0 .
$$

Now let $\left\{\xi_{n}\right\}_{n \in \mathbf{N}}$ be a sequence of $C^{1}$ nonnegative functions such that $\xi_{n}(x)=0$ if $x \leq 1 / 2 n, \xi_{n}(x)=1$ for $x \geq 1 / n$ and $\xi_{n}^{\prime} \geq 0$ for all $x>0$. Take also an arbitrary function $\varphi(t) \in C_{0}^{\infty}(\mathbf{R})$ such that $\varphi(t)$ is nonnegative and has support contained in the half line $t>0$. We then multiply both sides of (3.16) by $\xi_{n}(x) \varphi(t) \operatorname{sgn}^{+} g$, where $\operatorname{sgn}^{+} g=1$ when $g$ is positive and $\operatorname{sgn}^{+} g=0$ elsewhere. A quick computation reveals that

$$
\begin{aligned}
& \int_{0}^{\infty} \varphi(t) d t\left(\int_{0}^{\infty} \xi_{n} x^{2} g^{+} d x\right)_{t} \\
& \quad=\int_{0}^{\infty} \varphi(t) d t \int_{0}^{\infty} \xi_{n}\left(x^{4} g_{x}^{+}\right)_{x} d x \\
& +\int_{0}^{\infty} \varphi(t) d t \int_{0}^{\infty} \xi_{n}\left(x^{4} g^{+}\right)_{x} d x \\
& +\int_{0}^{\infty} \varphi(t) d t \int_{0}^{\infty} \xi_{n}\left(x^{4}\left(\left(f_{1}+f_{2}\right) g^{+}\right)\right)_{x} d x \\
& \quad \equiv I_{1}+I_{2}+I_{3} .
\end{aligned}
$$


One then has

$$
\begin{aligned}
& I_{3}=\int_{0}^{\infty} \varphi(t) d t \int_{0}^{\infty} \xi_{n}\left(x^{4}\left(\left(f_{1}+f_{2}\right) g^{+}\right)\right)_{x} d x \\
& =-\int_{0}^{\infty} \varphi(t) d t \int_{0}^{\infty} \xi_{n}^{\prime}\left(x^{4}\left(\left(f_{1}+f_{2}\right) g^{+}\right)\right) d x \leq 0,
\end{aligned}
$$

and similarly,

$$
I_{2} \leq 0
$$

On the other hand,

$$
\begin{aligned}
& I_{1}=\int_{0}^{\infty} \varphi(t) d t \int_{0}^{\infty} \xi_{n}\left(x^{4} g_{x}^{+}\right)_{x} d x \\
& =\int_{0}^{\infty} \varphi(t) d t \int_{0}^{\infty}\left(x^{4} \xi_{n}^{\prime}\right)^{\prime} g^{+} d x \\
& =\int_{0}^{\infty} \varphi(t) d t \int_{1 / 2 n}^{1 / n}\left(x^{4} \xi_{n}^{\prime \prime}+4 x^{3} \xi_{n}^{\prime}\right) g^{+} d x .
\end{aligned}
$$

Since $\left|\xi_{n}^{\prime}\right| \leq C n$ and $\left|\xi_{n}^{\prime \prime}\right| \leq C n^{2}$, for some generic constant $C>0$, (1.10a) we then have by

$$
x^{4}\left|\xi_{n}^{\prime \prime}\right| g^{+} \leq C x^{4} n^{2} g^{+}(x, t) \leq C x^{2} g^{+}(x, t) \leq C, \quad \text { for all } x \in\left(\frac{1}{2 n}, \frac{1}{n}\right),
$$

and, in the same way,

$$
x^{3}\left|\xi_{n}^{\prime}\right| g^{+}(x, t) \leq C x^{3} n g^{+}(x, t) \leq C x^{2} g^{+}(x, t) \leq C, \quad \text { for all } x \in\left(\frac{1}{2 n}, \frac{1}{n}\right) .
$$

Therefore,

$$
I_{1} \longrightarrow 0, \quad \text { as } n \longrightarrow \infty,
$$

and so, for every $\varphi \in C_{0}^{\infty}$, nonnegative with compact support in $t>0$,

$$
\int_{0}^{\infty} \varphi(t) d t\left(\int_{0}^{\infty} \xi_{n} x^{2} g^{+} d x\right)_{t} \leq 0,
$$

whence

$$
\frac{\partial}{\partial t} \int_{0}^{\infty} x^{2}\left(f_{1}-f_{2}\right)^{+} d x \leq 0
$$

and thus $f_{1} \equiv f_{2}$.

End of the proof of Theorem 1. We take up here the notation in Lemma 2.1, and observe that by assumption the initial data $F$ satisfies

$$
F(x) \leq \frac{C}{x^{b+1}}
$$

for some $b>3$, so that $x^{4} F(x) \longrightarrow 0$ as $x \longrightarrow+\infty$. Therefore, the uniqueness result in Theorem 2 can be applied to the solution $v$ constructed therein that satisfies (1.11a) and (1.11b).

\section{Singularity PRofiles: matched ASymptotic EXPANSiOns}

We explain in this section how the existence of the solutions described in Theorem 3 is derived in a formal way. 
1.1. Preliminary steps. Our starting point is the change of variables (2.1), which transforms (1.1a) into (2.2). A first important remark is that as $y \longrightarrow-\infty$, the dominant terms in (2.2) are

$$
v_{t}=v_{y y}+v_{y}-2 v+2 v^{2}+2 v v_{y}, \quad \text { for } y \in(-\infty,+\infty) \text { and } t>0 .
$$

Moreover, a dominated balance argument reveals that when $v \gg 1$, the dynamics of (4.1) is encoded in the simplified equation

$$
v_{t}=v_{y y}+2 v v_{y}+2 v^{2} .
$$

We shall therefore consider our rescaled equation (2.2) as a sort of perturbation of (4.2) above. It has been shown in [AE] that (4.2) has solutions which blow-up in finite time. By this we mean that there exist $T>0$ and sequences $\left\{y_{n}\right\}_{n \in \mathbf{N}},\left\{t_{n}\right\}_{n \in \mathbf{N}}$ such that $\lim _{n \longrightarrow \infty} y_{n}=y_{0}$, with $-\infty \leq y_{0} \leq+\infty, \lim _{n} \longrightarrow \infty t_{n}=T$ and $\lim _{n \longrightarrow \infty} v\left(y_{n}, t_{n}\right)=\infty$. It was also proved in [AE] that, if blow-up occurs for a solution $v(y, t)$ of $(4.2)$ such that $v(y, 0)$ decays exponentially fast as $y \longrightarrow+\infty$, then the singularity must unfold at $y=-\infty$. The dynamics of the singularity generation should be driven by the equation

$$
v_{t}=2 v v_{y}+2 v^{2} \text {. }
$$

Indeed, dominance of $v_{y y}$ and $2 v v_{y}$ would lead to a flow described by the Burgers equation, which does not develop singularities at all. On the other hand, should the terms $v_{y y}$ and $v^{2}$ prevail in (4.2), one would be led to a scenario described by the semilinear equation

$$
v_{t}=v_{y y}+2 v^{2} .
$$

This case is now well understood (cf. for instance [HV1], [V1]). In particular, it is known that when $v(y, 0)$ decays mildly as $y \longrightarrow \pm \infty$, blow-up always occurs in a bounded space region. Since we are looking for a convection-driven blow-up occurring at $y=-\infty$ in our case, we have to exclude this last possibility of our current discussion. Finally, if all three terms on the right of (4.2) were of the same order near blow-up, a quick dimensional analysis would yield that $v$ should be bounded.

A question that naturally arises is the manner of blow-up for equation (4.3). This problem has been considered in $[\mathrm{NT}]$. The authors construct therein solutions of (4.3) for the corresponding Riemann problem which exhibit a shock $\lambda(t)$ propagating to the left in the form

$$
\lambda(t) \sim \log \left(1-\frac{t}{T}\right)
$$

Near such a shock, a singularity unfolds that satisfies

$$
v(t)=O\left(\frac{1}{T-t}\right) .
$$

Taking these facts into account, it seems natural to rescale again as follows

$$
v(y, t)=(T-t)^{-1} \phi(\xi, \tau), \quad \xi=y-\log (T-t), \tau=-\log (T-t) .
$$

In this way, (2.4) is transformed into the following equation

$$
\begin{aligned}
\phi_{\tau} & =e^{-\tau}\left(\phi_{\xi \xi}+\phi_{\xi}-2 \phi\right)+(2 \phi-1)\left(\phi+\phi_{\xi}\right)+e^{-2 \tau+\xi}\left(3 \phi+\phi_{\xi}\right) \\
& \equiv e^{-\tau} \phi_{\xi \xi}+(2 \phi-1)\left(\phi+\phi_{\xi}\right)+h(\xi, \tau),
\end{aligned}
$$




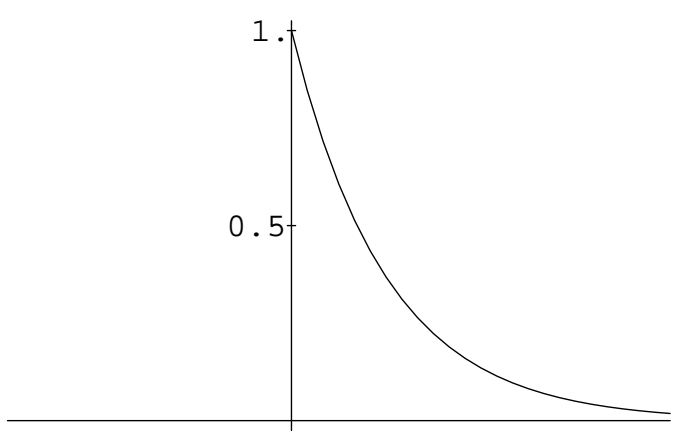

FiguRE 1

for $-\infty<\xi<\infty$ and $-\log T<\tau<\infty$, where

$$
h(\xi, \tau) \equiv e^{-\tau}\left(\phi_{\xi}-2 \phi\right)+e^{-2 \tau+\xi}\left(3 \phi+\phi_{\xi}\right) .
$$

For definiteness, in what follows we shall normalize the blow-up time by taking $T=1$. It seems sensible to expect that, as $\tau \longrightarrow \infty$, solutions of (4.5) will converge towards suitable solutions of the associated stationary equation, namely

$$
(2 \phi-1)\left(\phi+\phi_{\xi}\right)=0
$$

Notice that (4.6) allows for discontinuous solutions, provided that some balance conditions are met at the corresponding jumps. Summing up our previous remarks, we may now state a first requirement to be met by our solutions:

We shall look for solutions of (1.1) which blow up at $t=1$, and that satisfy the following property: When rescaled by means of (2.3) and (2.4), the scaled function $\phi(\xi, \tau)$ converges as $\tau \longrightarrow \infty$ to a bounded solution of (4.6) which may have a nonempty set $S$ of discontinuity points. At any $\xi \in S$, we shall require the following Rankine-Hugoniot conditions to hold:

$$
\left[\phi^{2}-\phi\right] \equiv\left(\phi^{2}\left(\xi^{+}\right)-\phi\left(\xi^{+}\right)\right)-\left(\phi^{2}\left(\xi^{-}\right)-\phi\left(\xi^{-}\right)\right)=0,
$$

where, as customary, $\phi\left(\xi^{+}\right)=\lim _{r} \longrightarrow \xi^{+} \phi(r)$ and $\phi\left(\xi^{-}\right)=\lim _{r} \longrightarrow \xi^{-} \phi(r)$, and an entropy condition

$$
\phi^{\prime}(\xi) \geq-C, \quad \text { in the sense of measures, for some positive constant } C .
$$

One example of such a solution of (4.6) is given in Figure 1.

In other words, as $\tau \longrightarrow \infty$ we expect $\phi(\xi, \tau)$ to converge towards a suitable entropic solution of (4.6) due to the vanishing diffusivity in (4.5). Such functions will therefore provide the final blow-up patterns for the solutions referred to in Theorem 1. It is therefore natural to ask how such limit profiles could be characterized. We shall address this question under the assumption that the discontinuity set $S$ consists of a finite number of points only

$$
S=\left\{\xi_{1}, \xi_{2}, \cdots, \xi_{N}\right\} .
$$

A solution $\bar{\phi}(\xi)$ of $(4.6)-(4.9)$ is then determined by specifying the set of points $x_{1}, x_{2}, \cdots, x_{N+1}$ where $\bar{\phi}\left(x_{i}\right)=1 / 2$. Indeed, away from such points, $\bar{\phi}(\xi)$ is of exponential type, i.e., $\bar{\phi}=C e^{-\xi}$ in every continuity interval $\left(\xi_{i}, \xi_{i+1}\right)$. Note that 
in Figure 1, $x_{1}$ has been selected so that $\bar{\phi}(0)=1$. For definiteness we shall retain this assumption for the rest of the paper. Condition (4.8) now reads

$$
[\phi]>0 \quad \text { at } \xi=\xi_{i}, \quad i=1,2, \cdots, N .
$$

In turn, (4.7) yields

$$
\bar{\phi}\left(\xi_{i}^{+}\right)-\frac{1}{2}=\frac{1}{2}-\bar{\phi}\left(\xi_{i}^{-}\right), \quad i=1,2, \cdots, N .
$$

4.2. Shock-induced instability of the final profiles. When looking for timedependent solutions of (1.1) which behave as indicated above, an important feature to be reckoned with is the unstable character of the blow-up patterns $\bar{\phi}$. By this we refer to a property shared by many blow-up problems, that stems from the fact that slight changes in the initial values give rise to variations in the value of the blow-up time as well as in the location of the blow-up points (cf. for instance [HV2] for an analysis of these facts for a semilinear heat equation). One of the main features of this paper is that in the case under examination here, this type of instability is related to the existence of shocks for the hyperbolic part of (4.5). To be more precise, let $\bar{\phi}$ be the solution of (4.6) given by

$$
\bar{\phi}(\xi)= \begin{cases}C_{1} e^{-\xi} & \text { if } \xi>0, \\ C_{2} e^{-\xi} & \text { if } \xi<0 .\end{cases}
$$

In this case the shock condition reads

$$
C_{1}-\frac{1}{2}=\frac{1}{2}-C_{2}, \quad C_{1}>C_{2}
$$

Consider now the solution of the equation

$$
\phi_{\tau}=(2 \phi-1)\left(\phi+\phi_{\xi}\right), \quad \text { for } \quad \xi \in(0, \infty), \tau>0,
$$

with the following perturbation of $\bar{\phi}$ as initial data

$$
\phi(\xi, 0)= \begin{cases}C_{1} e^{-\xi} & \text { if } \xi>\lambda(0), \\ C_{2} e^{-\xi} & \text { if } \xi<\lambda(0) .\end{cases}
$$

with $\lambda(0)$ a fixed arbitrary positive constant. Let $\lambda(\tau)$ be the point such that $\phi(\lambda(\tau), \tau)=1 / 2$. The Rankine-Hugoniot condition now reads

$$
\begin{aligned}
-\frac{d \lambda}{d \tau} & =\phi\left(\lambda^{+}(\tau), \tau\right)+\phi\left(\lambda^{-}(\tau), \tau\right)-1 \\
& =C_{1} e^{-\lambda(\tau)}+C_{2} e^{-\lambda(\tau)}-1 .
\end{aligned}
$$

Using Taylor's theorem, we obtain

$$
\begin{aligned}
-\frac{d \lambda}{d \tau} & \sim C_{1}(1-\lambda(\tau))+C_{2}(1-\lambda(\tau))-1 \\
& =-\left(C_{1}+C_{2}\right) \lambda(\tau),
\end{aligned}
$$

or equivalently

$$
\frac{d \lambda}{d \tau} \sim \lambda(\tau)
$$

An exponential instability would then arise for arbitrarily small perturbations of the stationary solution $\bar{\phi}$. 


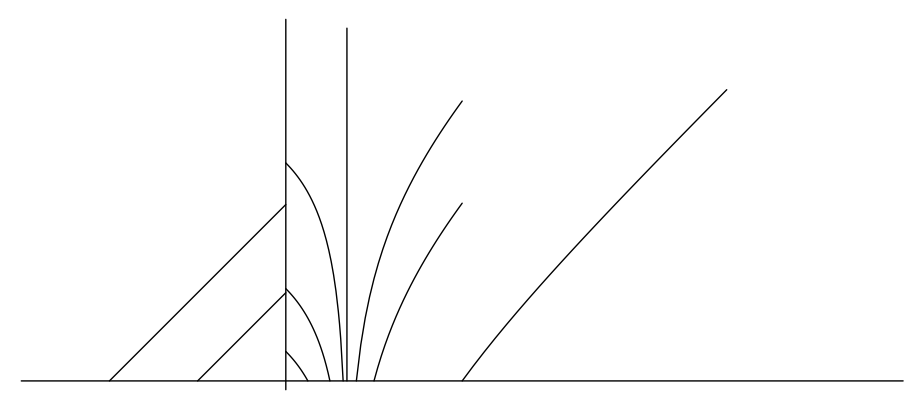

Figure 2

4.3. The asymptotics away from the shocks. While the dynamics of (4.5) is basically driven by the hyperbolic equation (4.14), there are two regions where diffusive effects are important. One of these is obviously the neighborhood of the unfolding shocks. The other one is the region where the factor $(2 \bar{\phi}-1)$ in (4.6) vanishes and $\bar{\phi}^{\prime}<0$. In fact, these last points are very important in determining the asymptotic profile. This can be seen from an analysis of the characteristic curves of the equation obtained by linearizing in (4.14) around $\bar{\phi}$, namely

$$
G_{\tau}=(2 \bar{\phi}-1)\left(G+G_{\xi}\right) \text {. }
$$

Indeed, if $\bar{\phi}$ is as in (4.12) with $C_{1}=1$ and $C_{2}=0$, the characteristics of (4.19) are as depicted in Figure 2. In this figure $\bar{\phi}\left(x_{1}\right)=\frac{1}{2}$. This clearly shows that perturbations propagate from a neighborhood of $\xi=x_{1}$, where diffusive effects need to be understood. To this end, we again fix an index $i$ and introduce a new variable $\lambda$, given by

$$
\lambda=\left(\xi-x_{i}\right) e^{\tau / 2} .
$$

We now linearize around $\bar{\phi}$ by setting

$$
\varphi(\lambda, \tau)=\phi\left(x_{i}+\lambda e^{-\tau / 2}, \tau\right)-\bar{\phi}\left(x_{i}+\lambda e^{-\tau / 2}\right) .
$$

A straightforward computation reveals that $\varphi$ solves

$$
\begin{aligned}
\varphi_{\tau}= & \varphi_{\lambda \lambda}-\frac{3}{2} \lambda \varphi_{\lambda}+e^{-\tau}\left(\bar{\phi}_{\xi \xi}+\bar{\phi}_{\xi}-2 \bar{\phi}\right)+(2 \bar{\phi}-1) \varphi+e^{\tau / 2} \varphi_{\lambda}\left((2 \bar{\phi}-1)+\lambda e^{-\tau / 2}\right) \\
& +2 e^{\tau / 2} \varphi \varphi_{\lambda}+2 \varphi^{2}+\left(e^{-\tau / 2} \varphi_{\lambda}-2 e^{-\tau} \varphi\right)+2 e^{\xi-2 \tau} \bar{\phi}+e^{\xi-2 \tau}\left(3 \varphi+e^{\tau / 2} \varphi_{\lambda}\right) \\
\equiv & \varphi_{\lambda \lambda}-\frac{3}{2} \lambda \varphi_{\lambda}+e^{-\tau}\left(\bar{\phi}_{\xi \xi}+\bar{\phi}_{\xi}-2 \bar{\phi}\right)+(2 \bar{\phi}-1)\left(\varphi+e^{\tau / 2} \varphi_{\lambda}\right)+g_{1}(\lambda, \tau)
\end{aligned}
$$

where

$$
\begin{aligned}
g_{1}(\lambda, \tau) \equiv & \lambda \varphi_{\lambda}+2 \varphi^{2}+2 e^{\tau / 2} \varphi \varphi_{\lambda}-2 e^{-\tau} \varphi+e^{-\tau / 2} \varphi_{\lambda} \\
& +2 e^{-2 \tau+\xi} \bar{\phi}+e^{-2 \tau+\xi}\left(3 \varphi+e^{\tau / 2} \varphi_{\lambda}\right)
\end{aligned}
$$

To take proper account of the third and fourth terms in the right of (4.22), we set

$$
\varphi(\lambda, \tau)=e^{-\tau} \phi_{1}(\xi)+\psi(\lambda, \tau),
$$


where $\phi_{1}(\xi)$ solves

$$
\phi_{1}+\left(\bar{\phi}_{\xi \xi}+\bar{\phi}_{\xi}-2 \bar{\phi}\right)+(2 \bar{\phi}-1)\left(\phi_{1}+\phi_{1 \xi}\right)=0
$$

In other words, we are replacing (4.21) by

$$
\phi\left(x_{i}+\lambda e^{-\tau / 2}, \tau\right)=\bar{\phi}\left(x_{i}+\lambda e^{-\tau / 2}\right)+e^{-\tau} \phi_{1}\left(x_{i}+\lambda e^{-\tau / 2}\right)+\psi(\lambda, \tau) .
$$

It turns out that $\psi$ satisfies the equation

$$
\psi_{\tau}=\psi_{\lambda \lambda}-\frac{3}{2} \lambda \psi_{\lambda}+g(\lambda, \tau) \equiv A \psi+g(\lambda, \tau)
$$

where

$$
\begin{aligned}
& g(\lambda, \tau)=e^{-2 \tau} \phi_{1 \xi \xi}-\frac{1}{2} \lambda e^{-3 \tau / 2} \phi_{1 \xi}+e^{-2 \tau} \phi_{1 \xi}+\lambda \psi_{\lambda}+e^{-\tau / 2} \psi_{\lambda} \\
& +2 \varphi^{2}+2 e^{\tau / 2} \varphi \varphi_{\lambda}+2 e^{-2 \tau+\xi} \bar{\phi}+e^{-2 \tau+\xi}\left(3 \varphi+e^{\tau / 2} \varphi_{\lambda}\right) .
\end{aligned}
$$

Equation (4.26) can be seen as a semilinear evolution equation in a suitable function space, which is selected in view of the spectral properties of the operator $A$. This last is easily seen to be a self-adjoint operator in the space $L_{w}^{2}(\mathbf{R})$ given by

$$
L_{w}^{2}(\mathbf{R})=\left\{f \in L_{\mathrm{loc}}^{1}(\mathbf{R}) ; \quad \int_{\mathbf{R}}|f(r)|^{2} e^{-r^{2} / 4} d r<\infty\right\} .
$$

The corresponding domain $D(A)$ is seen to coincide with the space

$$
H_{w}^{2}(\mathbf{R})=\left\{f \in L_{\mathrm{loc}}^{1}(\mathbf{R}) ; \quad f, f^{\prime} \text { and } f^{\prime \prime} \text { belong to } L_{w}^{2}(\mathbf{R})\right\} .
$$

Clearly, $L_{w}^{2}(\mathbf{R})$ is a Hilbert space when endowed with the norm

$$
\|f\|^{2}=\langle f, f\rangle=\int_{\mathbf{R}}|f(r)|^{2} e^{-r^{2} / 4} d r .
$$

A quick check reveals that $A$ has eigenvalues $\left\{\lambda_{k}\right\}$ given by

$$
\lambda_{k}=-\frac{3 k}{2}, \quad k=0,1,2, \cdots .
$$

The corresponding eigenfunctions, which can be written in terms of rescaled Hermite polynomials, will be denoted by

$$
L_{0}, L_{1}(\lambda), L_{2}(\lambda), \cdots
$$

where $L_{0}$ is constant, $L_{1}(\lambda)=a_{1} \lambda$, and the $L_{k}$ 's satisfy the normalization condition $\left\|L_{k}\right\|=1$ for every $k$. It is then natural to expand $\psi$ as a Fourier series

$$
\psi(\lambda, \tau)=\sum_{k=0}^{\infty} a_{k}(\tau) L_{k}(\lambda) .
$$

The coefficients $\left\{a_{k}(\tau)\right\}$ are to be determined by substituting (4.28) in (4.26). If $g(\lambda, \tau)$ in that equation consists of lower order terms, as it will be proved, we readily obtain that for $\tau \gg 1$,

$$
\left\{\begin{array}{l}
a_{0}(\tau) \sim a_{0} \quad \text { for some constant } a_{0}, \\
a_{1}(\tau) \sim C e^{-3 \tau / 2} \quad \text { for some constant } \quad C, \\
a_{k}(\tau)=o\left(e^{-3 \tau / 2}\right), \quad \text { uniformly on bounded sets of } \lambda .
\end{array}\right.
$$


Combining (4.29) and (4.25), we arrive at

$$
\begin{gathered}
\phi\left(x_{i}+\lambda e^{-\tau / 2}, \tau\right) \sim \bar{\phi}\left(x_{i}+\lambda e^{-\tau / 2}\right)+e^{-\tau} \phi_{1}\left(x_{i}+\lambda e^{-\tau / 2}\right) \\
+a_{0}+C \lambda e^{-3 \tau / 2}+o\left(e^{-3 \tau / 2}\right),
\end{gathered}
$$

uniformly for $\lambda$ on bounded subsets of $\mathbf{R}$ and $\tau \gg 1$. For definiteness, we shall assume in what follows that $i=1$, so that the function $\bar{\phi}$ in Figure 1 is such that

$$
\bar{\phi}(\xi)=e^{-\xi}, \text { for } 0<\xi<\xi_{1},
$$

and thus, $x_{1}=\log 2$. In that case, integrating the equation (4.24), we obtain

$$
\phi_{1}(\xi)=e^{-\left(\xi-x_{1}\right)}+C_{0}\left(1-e^{-\left(\xi-x_{1}\right)}\right),
$$

where $C_{0}$ is an arbitrary constant. When $\lambda$ remains bounded, $\lambda=O(1)$, and for large enough $\tau$, i.e. at points $\xi, \tau$ where $\left|\xi-x_{1}\right|=|\lambda| e^{-\tau / 2} \ll 1$, one then has

$$
\phi_{1}(\xi) \sim\left(1+C_{1}\right)-\lambda e^{-\tau / 2},
$$

with $C_{1}=C_{0} /\left(1-C_{0}\right)$. In view of (4.30) and (4.32), the following expansion holds

$$
\begin{gathered}
\phi(\xi, \tau) \sim a_{0}+e^{-\xi}+\left(1+C_{1}\right) e^{-\tau}-e^{-3 \tau / 2} \lambda+C e^{-3 \tau / 2} \lambda \\
\equiv a_{0}+e^{-\xi}+\left(1+C_{1}\right) e^{-\tau}+C_{2} e^{-3 \tau / 2} \lambda,
\end{gathered}
$$

for $\left|\xi-x_{1}\right|=|\lambda| e^{-\tau / 2}$, with $\lambda=O(1)$ and $\tau \gg 1$. Away from the smoothed shock, which we have assumed to occur near $\xi=0$, we expect for $\phi$ an expansion such as

$$
\begin{aligned}
\phi(\xi, \tau)= & \bar{\phi}(\xi)+e^{-\tau} \phi_{1}(\xi)+\cdots \\
& =e^{-\xi}+e^{-\tau}\left(e^{-\left(\xi-x_{1}\right)}\right)+C_{0}\left(1-e^{-\left(\xi-x_{1}\right)}\right)+\cdots .
\end{aligned}
$$

It is important to notice then that (4.33) can be matched to (4.34) provided that

$$
a_{0}=0 \text { in (4.33). }
$$

As a matter of fact, if $a_{0} \neq 0$, we could still remain close to a shifted version of $\bar{\phi}$, but not to $\bar{\phi}$ itself.

To describe the structure of the diffusive boundary layers introduced by the term $e^{-\tau} \phi_{\xi \xi}$ in equation (4.5) near the unfolding shocks, we take a fixed index $i$ and define an inner variable as follows

$$
\eta=\left(\xi-\xi_{i}\right) e^{\tau} .
$$

Then (4.5) transforms into

$$
\begin{aligned}
e^{-\tau} \phi_{\tau}=\phi_{\eta \eta}+(2 \phi-1) \phi_{\eta}+e^{-\tau}\left((2 \phi-1) \phi-\eta \phi_{\eta}+\phi_{\eta}\right) & \\
- & -2 e^{-2 \tau} \phi+e^{-3 \tau} e^{\xi_{i}+\eta e^{-\tau}}\left(3 \phi+e^{-\tau} \phi_{\eta}\right) .
\end{aligned}
$$

It then seems natural to expect that the dynamics near $\xi=\xi_{i}$ will be asymptotically described by a Burgers-type mechanism. Namely, for $|\eta|=O(1)$ and $\tau \gg 1$, we expect $\phi(\xi, \tau)$ to behave as the solution of

$$
\phi_{\eta \eta}+(2 \phi-1) \phi_{\eta}=0, \quad \text { for } \quad \eta \in(-\infty,+\infty), \tau>0,
$$

with boundary conditions

$$
\begin{aligned}
& \phi(\eta) \longrightarrow L_{l}=\bar{\phi}\left(\xi_{i}^{-}\right) \text {as } \eta \longrightarrow-\infty, \\
& \phi(\eta) \longrightarrow L_{r}=\bar{\phi}\left(\xi_{i}^{+}\right) \text {as } \eta \longrightarrow+\infty .
\end{aligned}
$$


The solution of (4.38) is explicitly given by

$$
\phi_{B}(\eta)=\frac{L_{r}+L_{l} e^{-\left(L_{r}-L_{l}\right)\left(\eta-\eta_{0}\right)}}{1+e^{-\left(L_{r}-L_{l}\right)\left(\eta-\eta_{0}\right)}}
$$

where

$$
L_{r}+L_{l}=1 \text {. }
$$

Notice that there are two free parameters in (4.39), say $L_{r}$ and $\eta_{0}$. These cannot be fixed yet, since they have to be selected by imposing a suitable matching with the region located away from the smoothed shocks. Let us now turn our attention to that region.

4.4. Matching the regions close to and away from the smoothed shocks. We now address the question of the compatibility of the asymptotic representations given in (4.34) an in (4.39). In particular, we can think of (4.34) as an outer expansion to be matched with an inner one near $\xi=0$. In terms of the variable $\eta=\xi e^{\tau}$ defined in (4.36) (remember that we have assumed that $\left.\xi_{0}=0\right),(4.34) \mathrm{can}$ be rewritten in the form

$$
\phi(\eta, \tau) \sim 1-\eta e^{-\tau}+\left(2-C_{0}\right) e^{-\tau}+o\left(e^{-\tau}\right), \quad \text { for } \eta>0, \eta=o\left(e^{\tau}\right), \tau \gg 1 .
$$

As to the corresponding condition for $\eta<0$, we may just assume that

$$
\phi(\eta, \tau)=O\left(e^{-\tau}\right), \quad \text { for } \eta<0, \eta=o\left(e^{\tau}\right), \tau \gg 1 .
$$

As an inner expansion, in (4.37) we shall try

$$
\phi(\eta, \tau)=\phi_{0}(\eta)+e^{-\tau} \phi_{1}(\eta)+\cdots,
$$

where

$$
\begin{aligned}
& \phi_{0}=\frac{1}{1+e^{-\left(\eta-\eta_{0}\right)}} \text { is such that } \\
& \phi_{0}(\eta) \longrightarrow 1 \text { as } \eta \longrightarrow \infty, \quad \phi_{0}(\eta) \longrightarrow 0 \quad \text { as } \quad \eta \longrightarrow-\infty .
\end{aligned}
$$

Notice that since $\phi_{B}$ given in (4.39) depends on two arbitrary constants, (4.43) still leaves one parameter free, say $\eta_{0}$, in the definition of $\phi_{0}$. Plugging (4.42) into (4.37) and retaining leading order terms, we readily see that $\phi_{1}$ has to satisfy the equation

$$
\begin{aligned}
& \phi_{\eta \eta}+\left(2 \phi_{0}-1\right) \phi_{\eta}+2 \phi_{0 \eta} \phi=-\left(\left(2 \phi_{0}-1\right) \phi_{0}-\eta \phi_{0 \eta}+\phi_{0 \eta}\right) \\
& =-\left(\left(2 \phi_{0}-1\right) \phi_{0}+\phi_{0 \eta}-\left(\eta-\eta_{0}\right) \phi_{0 \eta}-\eta_{0} \phi_{0_{\eta}}\right) \\
& \equiv F_{1}\left(\eta-\eta_{0}\right)+\eta_{0} F_{2}\left(\eta-\eta_{0}\right) .
\end{aligned}
$$

Let us describe in detail the behaviour of the solutions of (4.44). To begin with, consider the homogeneous equation

$$
\phi_{\eta \eta}+\left(2 \phi_{0}-1\right) \phi_{\eta}+2 \phi_{0}^{\prime} \phi=0 .
$$

This is nothing but the linearization around the solution $\phi_{0}$ of the equation (4.38a). On the other hand, as we already noticed, the set of solutions of such an equation consists of the following two-parameter family

$$
\phi(\eta ; a, b)=\frac{a+(1-a) e^{-(2 a-1)(\eta-b)}}{1+e^{-(2 a-1)(\eta-b)}},
$$


where $a$ represents the height of $\phi$ as $\eta \longrightarrow \infty$ (so that $(1-$ a) corresponds to the height as $\eta \longrightarrow-\infty)$, and $b$ is a translation parameter, so that $\phi(\eta ; a, b)=$ $\phi(\eta-b, a, 0)$. It then turns out that

$$
\phi_{1,1}(\eta)=\left.\frac{\partial \phi}{\partial a}\right|_{a=1}=\frac{1+2(\eta-b) e^{-(\eta-b)}-e^{-2(\eta-b)}}{\left(1+e^{-(\eta-b)}\right)^{2}}
$$

and

$$
\phi_{1,2}(\eta)=\left.\frac{\partial \phi}{\partial b}\right|_{a=1}=\frac{e^{-(\eta-b)}}{\left(1+e^{-(\eta-b)}\right)^{2}} .
$$

are two independent solutions of (4.45). A direct computation then shows that

$$
\begin{aligned}
\phi_{1,1}(\eta) \longrightarrow 1 \quad \text { as } & \eta \longrightarrow+\infty ; \quad \phi_{1,1} \longrightarrow-1 \quad \text { as } \eta \longrightarrow-\infty, \\
& \phi_{1,2}(\eta) \sim e^{-(\eta-b)} \quad \text { as } \eta \longrightarrow+\infty \\
& \phi_{1,2} \sim e^{(\eta-b)} \quad \text { as } \eta \longrightarrow-\infty .
\end{aligned}
$$

As a next step we now describe the asymptotics of those solutions $\phi$ of (4.44) such that $\phi(0)=\phi^{\prime}(0)=0$. In view of the explicit form of the right hand side in (4.44) and since the equation is linear, we have $\phi\left(\eta, \eta_{0}\right)=\bar{\phi}_{1}\left(\eta-\eta_{0}\right)+\eta_{0} \bar{\phi}_{2}\left(\eta-\eta_{0}\right)$, where $\bar{\phi}_{i}$ satisfies (4.44) with nonhomogeneous term $F_{i}$ for $i=1,2$. Using the variation of constants formula, we readily obtain

$$
\begin{gathered}
\bar{\phi}_{1}\left(\eta-\eta_{0}\right) \sim\left\{\begin{array}{l}
-\left(\eta-\eta_{0}\right) \Gamma_{1} \quad \text { as } \quad \eta \longrightarrow+\infty, \\
\gamma_{1} \quad \text { as } \eta \longrightarrow-\infty,
\end{array}\right. \\
\bar{\phi}_{2}\left(\eta-\eta_{0}\right) \sim\left\{\begin{array}{lll}
\Gamma_{2} & \text { as } & \eta \longrightarrow+\infty, \\
\gamma_{2} & \text { as } & \longrightarrow
\end{array},-\infty,\right.
\end{gathered}
$$

where $\Gamma_{1}, \Gamma_{2}, \gamma_{1}$ and $\gamma_{2}$ are fixed constants independent of $\eta_{0}$. It then turns out that the general solution $\phi_{1}$ of (4.44) satisfies

$$
\begin{gathered}
\phi_{1}\left(\eta, \eta_{0}\right) \sim C_{1}+O\left(e^{-\eta}\right)-\left(\eta-\eta_{0}\right)+\Gamma_{1}+\Gamma_{2} \eta_{0} \quad \text { as } \quad \eta \longrightarrow \infty, \\
\phi_{1}\left(\eta, \eta_{0}\right) \sim-C_{1}+O\left(e^{\eta}\right)+\gamma_{1}+\gamma_{2} \eta_{0} \quad \text { as } \quad \eta \longrightarrow-\infty
\end{gathered}
$$

where $C_{1}$ is an arbitrary constant. From (4.42) and (4.48) we obtain that the inner expansion for $\phi$ has the form

$$
\begin{gathered}
\phi(\eta, \tau) \sim 1+e^{-\tau}\left(C_{1}+\Gamma_{1}+\Gamma_{2} \eta_{0}-\left(\eta-\eta_{0}\right)\right) \quad \text { as } \eta \longrightarrow+\infty, \\
\phi(\eta, \tau) \sim e^{-\tau}\left(-C_{1}+\gamma_{1}+\gamma_{2} \eta_{0}\right) \quad \text { as } \eta \longrightarrow-\infty,
\end{gathered}
$$

Finally, matching (4.49) with (4.40) requires that

$$
\begin{gathered}
C_{1}=\gamma_{1}+\gamma_{2} \eta_{0}, \\
2-C_{0}=C_{1}+\Gamma_{1}+\eta_{0}(\Gamma+2+1),
\end{gathered}
$$

and this gives the values of the constants $C_{1}$ and $\eta_{0}$. Observe that at this stage the constant $C_{0}$ remains free. In order to fix its value the next term in the asymptotic expansion is needed in the same way as we have done to determine the value of $\eta_{0}$.

It might thus appear as if our problem had already been solved, at least at the current formal level. Indeed, we now have a candidate for our limit pattern. This consists of a Burgers-type profile near the smoothed shocks, given by $\phi(\xi, \tau)$ satisfying (4.42)-(4.44), which connects points far away from $\xi_{i}$ with $\phi(\xi, \tau)$ given by 
(4.34). Once such a global representation $\bar{\phi}(\xi, \tau)$ has been obtained, a natural way to prove the existence of our solutions would be to start from a profile close enough to such a function $\bar{\phi}(\xi, \tau)$ at, say, $\tau=\tau_{0}$, and then show that the corresponding solution of (4.5) would converge to $\bar{\phi}$ as $\tau \longrightarrow \infty$, where $\bar{\phi}$ is the function specified in (4.12).

As it turns out, however, the proposed profile $\bar{\phi}(\xi, \tau)$ has yet to be modified, due to a strong instability that is inherent to the problem, but that has been overlooked in the previous argument. In order to show the instability of the unfolding shocks, let us start with initial data as depicted in Figure 1 with a shock at $\xi=0$. It will be better to use an expansion of $\phi$ in the form

$$
\phi(\eta, \tau)=\phi_{0}(\eta-g(\tau))+e^{-\tau} \phi_{1}(\eta-g(\tau))+\cdots
$$

with $\eta=\xi e^{\tau}$, and where $g(\tau)$ is such that

$$
\phi(g(\tau), \tau)=\frac{1}{2}
$$

so that

$$
\left\{\begin{array}{l}
\phi_{0}(0)=\frac{1}{2}, \\
\phi_{k}(0)=0, \quad \text { for all } k=1,2, \cdots .
\end{array}\right.
$$

To the leading order, equation (4.37) reads

$$
e^{-\tau} \phi_{\tau}=\phi_{\eta \eta}+(2 \phi-1) \phi_{\eta}+e^{-\tau}\left((2 \phi-1) \phi-\eta \phi_{\eta}+\phi_{\eta}\right)
$$

whence

$$
-g^{\prime}(\tau) \phi_{0}^{\prime}=\phi_{1}^{\prime \prime}+\left(2 \phi_{0}-1\right) \phi_{1}^{\prime}+2 \phi_{0}^{\prime} \phi_{1}+\left(2 \phi_{0}-1\right) \phi_{0}-\eta \phi_{0}^{\prime}+\phi_{0}^{\prime} .
$$

Now let us call $W$ the solution of the problem

$$
\begin{aligned}
& W^{\prime \prime}(\eta)+\left(2 \phi_{0}(\eta-g(\tau))-1\right) W^{\prime}(\eta)+2 \phi_{0}^{\prime}(\eta-g(\tau)) W(\eta) \\
& +\left(\left(2 \phi_{0}(\eta-g(\tau))-1\right) \phi_{0}(\eta-g(\tau))-\eta \phi_{0}^{\prime}(\eta-g(\tau))+\phi_{0}^{\prime}(\eta-g(\tau))\right)=0 \\
& W(0)=0, \quad W(\eta)=o(1), \quad \text { as } \eta \longrightarrow-\infty
\end{aligned}
$$

It is straightforward to see that

$$
W(\eta)=-\eta+C_{\infty} \quad \text { as } \eta \longrightarrow+\infty,
$$

for some given constant $C_{\infty}$. On the other hand, let $V$ be the solution of

$$
\begin{aligned}
& V^{\prime \prime}(\eta)+\left(2 \phi_{0}(\eta)-1\right) V^{\prime}(\eta)+2 \phi_{0}^{\prime}(\eta) V(\eta)=\phi_{0}^{\prime}(\eta), \\
& V(0)=0, \quad V(\eta)=o(1) \quad \text { as } \eta \longrightarrow-\infty,
\end{aligned}
$$

We can then write

$$
\phi_{1}(\eta-g(\tau))=W(\eta, \tau)-g^{\prime}(\tau) V(\eta-g(\tau))+C(\tau) \phi_{1,1}(\eta-g(\tau)),
$$

where the last term in the right hand side is a solution of the homogeneous equation (4.45), and $C(\tau)$ is a constant which may possibly depend on $\tau$. In view of the definition of $W$ it is natural to split $W$ as follows

$$
W(\eta, \tau)=g(\tau) V(\eta-g(\tau))+R(\eta-g(\tau))
$$


where $R$ is a solution of

$$
\begin{aligned}
& R^{\prime \prime}(\eta)+\left(2 \phi_{0}(\eta)-1\right) R^{\prime}(\eta)+2 \phi_{0}^{\prime}(\eta) R(\eta), \\
& =-\left(\left(2 \phi_{0}(\eta)-1\right) \phi_{0}(\eta)-\eta \phi_{0}^{\prime}(\eta)+\phi_{0}^{\prime}(\eta)\right), \\
& R(0)=0, \quad R(\eta)=o(1) \quad \text { as } \eta \longrightarrow-\infty .
\end{aligned}
$$

Summing all these things up, we may represent $\phi_{1}$ in the form

$$
\begin{aligned}
\phi_{1}(\eta-g(\tau))= & -g^{\prime}(\tau) V(\eta-g(\tau))+R(\eta-g(\tau)) \\
& +C_{1}(\tau) \phi_{1,1}(\eta-g(\tau))+g(\tau) V(\eta-g(\tau))+\cdots .
\end{aligned}
$$

Taking into account the behaviour of the auxiliary functions $V, \phi_{1,1}$ and $R$, we obtain the following behaviours at $\pm \infty$

$$
\phi(\eta, \tau) \sim\left\{\begin{array}{l}
0-C_{1} e^{-\tau} \quad \text { as } \eta \longrightarrow-\infty \\
1+e^{-\tau}\left\{-g^{\prime}(\tau) V(+\infty)-(\eta-g(\tau))+C_{\infty}+C_{1}+g(\tau) V(\infty)\right\} \\
\text { as } \eta \longrightarrow \infty
\end{array}\right.
$$

We now have to match these conditions with the outer expansion given by (4.40). This gives $C_{1}=0$ and

$$
-g^{\prime}(\tau) V(+\infty)+g(\tau)+C_{\infty}+g(\tau) V(+\infty)=\left(2-C_{0}\right) .
$$

Now let us determine $V(+\infty)$. To this end we first remark that the function

$$
\phi_{c}(\eta)=\frac{1-c}{(1-2 c) e^{-(1-c) \eta}+1}
$$

satisfies

$$
\begin{aligned}
& \phi_{c}^{\prime \prime}+\left(2 \phi_{c}-1\right) \phi_{c}^{\prime}+c \phi_{c}^{\prime}=0 \\
& \phi_{c}(0)=\frac{1}{2}, \quad \phi_{c}(\eta) \longrightarrow 0, \quad \text { as } \eta \longrightarrow-\infty
\end{aligned}
$$

Therefore

$$
\begin{aligned}
& \frac{\partial \phi_{c}{ }^{\prime \prime}}{\partial c}+\left(2 \phi_{c}-1\right) \frac{\partial \phi_{c}{ }^{\prime}}{\partial c}+2 \phi_{c}^{\prime} \frac{\partial \phi_{c}}{\partial c}=-\phi_{c}^{\prime}-c \phi_{c}^{\prime} \\
& \frac{\partial \phi_{c}}{\partial c}(0)=0 ; \quad \frac{\partial \phi_{c}}{\partial c}(\eta) \longrightarrow 0, \quad \text { as } \eta \longrightarrow-\infty ; \quad \frac{\partial \phi_{c}}{\partial c}(\eta) \longrightarrow-1, \quad \text { as } \eta \longrightarrow \infty
\end{aligned}
$$

By the definition of $V$ we see that

$$
V(\eta)=-\left.\frac{\partial \phi_{c}}{\partial c}\right|_{c=0}
$$

and so $V(+\infty)=1$. Finally, the position of the unfolding shock $g(\tau)$ satisfies

$$
g^{\prime}(\tau)=2 g(\tau)+C_{\infty}-\left(2-C_{0}\right)
$$

and exhibits an exponential instability. This renders the matching of the inner solution with the outer solution impossible unless we select the positions of the smoothed shock $\lambda(\tau)$ where $\phi(\lambda(\tau), \tau)=1 / 2$ in a suitable way so as to cancel the exponential growth in (4.55). More precisely, we need to impose the requirement

$$
g\left(\tau_{0}\right)=-\int_{\tau_{0}}^{\infty} e^{-2 s}\left(C_{\infty}-2+C_{0}\right) d s=\frac{1}{2}\left(C_{\infty}-2+C_{0}\right) e^{-2 \tau_{0}} .
$$

Summing up, we are led to restate our basic goal in the following form 
We look for solutions of (1.1) that blow-up at $t=1$ and converge (in rescaled variables) to a given function $\bar{\phi}$ as $\tau=-\log (1-t) \longrightarrow \infty$. To this end, we start from a profile $\phi\left(\xi, \tau_{0}\right)$ described as follows. There will be $N$ points $\bar{x}_{i}$ where $\phi\left(\bar{x}_{i}, \tau_{0}\right)=$ $1 / 2$ and $\phi_{\xi}\left(\bar{x}_{i}, \tau_{0}\right)<0$, and $N$ points $\lambda_{1}(\tau), \cdots, \lambda_{N}(\tau)$ such that $\phi\left(\lambda_{i}\left(\tau_{0}\right), \tau_{0}\right)=1 / 2$ and $\phi_{\xi}\left(\lambda_{i}\left(\tau_{0}\right), \tau_{0}\right)>0$. The function $\phi\left(\xi, \tau_{0}\right)$ will be exponentially small in $\xi$ when $\xi \longrightarrow-\infty$, and points $\bar{x}_{i}$ (resp. $\lambda_{i}\left(\tau_{0}\right)$ ) will be selected close enough to points $x_{i}$ (resp. to points $\xi_{i}$ ) in the corresponding function $\bar{\phi}(\xi)$. The dynamics of the desired solutions proceeds as follows. On intervals $\left(\lambda_{i}(\tau), \lambda_{i+1}(\tau)\right), \phi(\xi, \tau)$ approaches a travelling wave solution $\phi(\eta-c(\tau), \tau)$, where $c(\tau)$ varies slowly in time. Away from such intervals, $\phi(\xi, \tau)$ is described by expansions of the type (4.34).

\section{Proof of the existence of Blowing up solutions}

This section is devoted to the proof of Theorem 3. To this end, we will make use of a topological argument which has some analogies with those described for instance in [HV2], [HV3] and [V2], where various singularity mechanisms for parabolic equations have been analyzed.

5.1. General description of the proof. To begin with, we take a fixed solution $\bar{\phi}$ of $(4.6)-(4.8)$ (cf. Figure 1). Such a function is uniquely determined by giving the set of points $x_{1}<x_{2}<\cdots<x_{N}$ where $\bar{\phi}\left(x_{i}\right)=1 / 2$. These in turn define the location of the $N$ discontinuity points $\xi_{0}, \xi_{1}, \cdots, \xi_{N-1}$ where we have

$$
\bar{\phi}\left(\xi^{+}\right)-\frac{1}{2}=\frac{1}{2}-\bar{\phi}\left(\xi^{-}\right)
$$

As in Section 4 , we take $x_{1}$ such that $\bar{\phi}(0)=1$ for simplicity.

5.1.1. A suitable functional frame. For given positive constants $M, \tau_{0}, \tau_{1}$ with $\tau_{1} \geq$ $\tau_{0}$, we now introduce a space of smooth, nonnegative functions $\mathcal{A}\left(M ; \tau_{0}, \tau_{1}\right)$ defined by the following conditions. For a function $\phi(\xi, \tau)$ to belong to $\mathcal{A}\left(M ; \tau_{0}, \tau_{1}\right)$, we require the existence of $N$ curves $\left\{\lambda_{1}(\tau), \lambda_{2}(\tau), \cdots, \lambda_{N}(\tau)\right\}$ of class $C^{1}$ such that, for every $i=1, \cdots, N$ and for all $\tau \in\left[\tau_{0}, \tau_{1}\right]$,

$$
\begin{gathered}
\left|\lambda_{i}(\tau)-\xi_{i}\right| \leq M e^{-\tau}, \\
\phi\left(\lambda_{i}(\tau), \tau\right)=\frac{1}{2}, \\
\phi_{\xi}\left(\lambda_{i}(\tau), \tau\right)>0 .
\end{gathered}
$$

The functions $\lambda_{i}(\tau)$ represent the location of the unfolding shocks. Moreover, these functions $\lambda_{i}(\tau)$ would move slowly, as required by the following condition

$$
\left|\frac{d \lambda_{i}}{d \tau}\right| \leq M e^{-\tau}
$$

Away from the curves $\left\{\lambda_{i}(\tau)\right\}$, the functions in the class will remain exponentially close to $\bar{\phi}$. More precisely, let $\phi_{1}$ be given in (4.34). We now require that

$$
\begin{aligned}
& \left|\phi(\xi, \tau)-\bar{\phi}(\xi)-e^{-\tau} \phi_{1}(\xi)\right| \leq M e^{-3 \tau / 2}\left(1+\left|\xi-x_{i}\right| e^{\tau / 2}\right) \\
& \text { for all } \xi \in\left(\xi_{i-1}, \xi_{i}\right) \text { such that } \max \left(\xi_{i-1}+2 M e^{-\tau}, \xi_{i}-M\right)<\xi<\xi_{i}-2 M e^{-\tau} \\
& \text { for } i=1, \cdots, N \text { and for all } \xi \in\left(\xi_{N}+2 M e^{-\tau}, 2 x_{N}\right) .
\end{aligned}
$$


Near the smoothed shocks, we only require the solution to be bounded as follows

$$
\phi(\xi, \tau) \leq M \text { for all } \xi \text { such that }\left|\xi-\xi_{i}\right| \leq 2 M e^{-\tau} \text { for some } i \text {, and all } \tau \in\left[\tau_{0}, \tau_{1}\right] \text {. }
$$

We have yet to make precise the expected behaviours for $\xi \longrightarrow \pm \infty$. These are required to be as follows:

$$
\begin{gathered}
\text { For all } \xi>2 x_{N} \text { and for all } \tau \in\left[\tau_{0}, \tau_{1}\right]: \\
\qquad \phi(\xi, \tau) \leq\left\{\begin{array}{l}
M e^{-\xi} \text { if } \xi \leq \tau-M, \\
M e^{2 \tau-3 \xi} \text { if } \xi \geq \tau-M .
\end{array}\right. \\
\phi(\xi, \tau) \leq M e^{-\tau} \text { for all } \xi \leq-2 M e^{-\tau} \text { and all } \tau \in\left[\tau_{0}, \tau_{1}\right] .
\end{gathered}
$$

It is clear that for any fixed $M \gg 1$ and $\tau_{0}>0$ the set $\mathcal{A}\left(M ; \tau_{0}, \tau_{1}\right)$ is not empty. If we take a function $\phi_{0}(\xi) \in \mathcal{A}\left(M / 2 ; \tau_{0}, \tau_{1}\right)$ and consider the Cauchy problem consisting of (4.5) for $\tau>\tau_{0}$ with initial condition $\phi\left(\xi, \tau_{0}\right)=\phi_{0}(\xi)$, standard continuous dependence results yield that the corresponding solution $\phi(\xi, \tau)$ remains in $\mathcal{A}\left(M ; \tau_{0}, \tau_{1}\right)$ provided that $\tau_{1}-\tau_{0}$ is small enough. We shall assume in the sequel that such a solution $\phi(\xi, \tau)$ is given, and prove that, if $\phi_{0}(\xi)$ is selected in a suitable way, and if $M$ and $\tau_{0}$ are large enough, the function $\phi(\xi, \tau)$ will actually remain in the interior of this class, say, $\phi(\xi, \tau) \in \mathcal{A}\left(M / 2 ; \tau_{0}, \tau_{1}\right)$, regardless of how large the difference $\left(\tau_{1}-\tau_{0}\right)$ may be. This will eventually allow us to extend such a solution as $\tau \longrightarrow \infty$, thus obtaining the global existence of $\phi$ in $\mathcal{A}\left(M ; \tau_{0},+\infty\right)$ and the required converge to $\bar{\phi}$. As a matter of fact, one considers $\phi_{0}$ as depending on $2 N+1$ parameters

$$
\phi_{0}(\xi)=\phi_{0}\left(\xi ; \tau_{0}, \alpha_{1}, \cdots, \alpha_{N}, k_{1}, \cdots, k_{N}\right)
$$

and show that our purposes may be achieved by selecting $\tau_{0}, \alpha_{1}, \cdots, \alpha_{N}, k_{1}, \cdots k_{N}$ in a suitable way.

5.1.2. Admisible Initial Data. For the sake of definiteness we shall only consider in detail the simplest case $N=1, \xi_{1}=0$ and $x_{1}=\log 2$. The case of a general solution $\bar{\phi}$ can be treated similarly with only minor modifications. We therefore select as $\bar{\phi}$ the following solution of (4.6)

$$
\bar{\phi}(\xi)=\left\{\begin{array}{l}
e^{-\xi} \text { if } \xi>0, \\
0 \quad \text { if } \xi>0 .
\end{array}\right.
$$

We shall consider functions $\phi_{0}$ depending on 3 parameters

$$
\phi_{0}(\xi)=\phi_{0}\left(\xi, \tau_{0}, \alpha, k\right) .
$$

Once we have defined the space $\mathcal{A}\left(M ; \tau_{0}, \tau_{1}\right)$ we now describe the set of functions which will be "admissible" initial data. This is done by prescribing in a precise way the behaviour of these functions near the points $x_{1}=\log 2$ and $\xi_{1}=0$. Let us fix $\zeta(\cdot)$ to be a regular nonnegative function of compact support in the interval $(0,2 M)$ and equal to one in some open subset $\mathcal{U}$ containing $x_{1}$. The function $\phi_{0}$ is an admissible initial data if for some $\tau_{1}>\tau_{0}, \phi \in \mathcal{A}\left(M / 2 ; \tau_{0}, \tau_{1}\right)$ and moreover the following requirements are satisfied. 
The behaviour away from the smoothed shocks. There exists a real number $\alpha$ such that for all $r$ satisfying $x_{1}+r e^{-\tau_{0} / 2} \in \mathcal{U}$ we have

$$
\phi_{0}\left(x_{1}+r e^{-\tau_{0} / 2}\right)=\bar{\phi}\left(x_{1}+r e^{-\tau_{0} / 2}\right)-e^{-\tau_{0}} \phi_{1}\left(x_{1}+r e^{-\tau_{0} / 2}\right)+\alpha \zeta\left(x_{1}+r e^{-\tau_{0} / 2}\right) .
$$

The behaviour near the smoothed shock $\xi=0$. There exist real numbers $\eta \equiv \eta_{0}\left(\tau_{0}\right)$ and $c\left(\tau_{0}\right)$ satisfying

$$
c\left(\tau_{0}\right)+\frac{1}{2} e^{\left(1-c\left(\tau_{0}\right)\right) \eta_{0}}=\frac{1}{2}
$$

with $\left|\eta_{0}\left(\tau_{0}\right)\right| \longrightarrow 0$ as $\tau_{0} \longrightarrow+\infty$, and such that

$$
\phi_{0}(\xi)=\frac{1-c\left(\tau_{0}\right)}{1+e^{\left(c\left(\tau_{0}\right)-1\right)\left(\left(\xi-\lambda\left(\tau_{0}\right)\right) e^{\left.\tau_{0}-\eta_{0}\right)}\right.}}
$$

for $\xi \in \mathcal{V}$, a small neighborhood of zero. Observe that one necesarily has that $\left|c\left(\tau_{0}\right)\right| \longrightarrow 0$ as $\tau_{0} \longrightarrow+\infty$. On the other hand, by definition

$$
\phi_{0}\left(\lambda\left(\tau_{0}\right)\right)=\frac{1-c\left(\tau_{0}\right)}{1+e^{\left(1-c\left(\tau_{0}\right)\right) \eta_{0}}}=\frac{1}{2} .
$$

We shall set $k=\lambda\left(\tau_{0}\right)$.

Observe that since $\phi_{0} \in \mathcal{A}\left(M / 2 ; \tau_{0}, \tau_{1}\right)$ for some $\tau_{1}>\tau_{0}$, we have in particular that $\phi_{0}$ satisfies (5.7). Therefore, by Theorem 2 , the corresponding initial data $F$ given by $x F(x)=\phi_{0}(\log x)$ is such that the equation (1.1a) has a unique global solution $f$ satisfying (1.11a), (1.11b) and (1.13) for all $t>0$.

5.1.3. Main steps in the proof. The following are the essential ingredients in our approach.

STEP ONE. Suppose that we choose any admisible initial data defined above, and the solution $\phi(\xi, \tau)$ belongs to the set $\overline{\mathcal{A}\left(M ; \tau_{0}, \tau_{1}\right)}$. If, moreover,

$$
l_{1}\left(\alpha, k ; \tau_{1}\right) \equiv \int_{\mathbf{R}} \Psi\left(r, \tau_{1}\right) e^{-r^{2} / 4} d r=0,
$$

where

$$
\Psi(r, \tau)=\phi_{0}\left(x_{1}+r e^{-\tau / 2}\right)-\bar{\phi}\left(x_{1}+r e^{-\tau / 2}\right)-e^{-\tau} \phi_{1}\left(x_{1}+r e^{-\tau / 2}\right),
$$

then the curve $\lambda(\tau)$ satisfies the ODE

$$
\frac{d \lambda}{d \tau}=\lambda(\tau)+C e^{-\tau}+\varepsilon h(\tau)
$$

for some positive constant $C$ independent of $M, \varepsilon=\varepsilon\left(\tau_{0}\right) \longrightarrow 0$ as $\tau_{0} \longrightarrow+\infty$, and some function $h(\tau)=O\left(e^{-\tau}\right)$ for $\tau \gg 1$.

STEP TWO. Assume that $\phi \in \overline{\mathcal{A}\left(M ; \tau_{0}, \tau_{1}\right)}$ and

$$
\begin{gathered}
l_{1}\left(\alpha, k ; \tau_{1}\right) \equiv \int_{\mathbf{R}} \Psi\left(r, \tau_{1}\right) e^{-r^{2} / 4} d r=0 \\
l_{2}\left(\alpha, k ; \tau_{1}\right) \equiv \lambda\left(\tau_{0}\right)+e^{-\tau_{0}} \int_{\tau_{0}}^{\tau_{1}} e^{-2 \sigma}(C+\varepsilon h(\sigma)) d \sigma=0 .
\end{gathered}
$$

Then $\phi(\xi, \tau) \in \mathcal{A}\left(M / 2 ; \tau_{0}, \tau_{1}\right)$. 
DEGREE ARGUMENT. Let us denote by $\phi(\xi, \tau ; \alpha, k)$ the solution with any admisible initial data at $\tau=\tau_{0}$ determined by the parameters $\alpha$ and $k$. For $\tau_{0} \leq \tau_{1}$ we define a subset of $\mathbf{R}^{2}$

$$
\mathcal{U}\left(\tau_{0}, \tau_{1}\right)=\left\{(\alpha, k) ; \phi(\xi, \tau ; \alpha, k) \in \mathcal{A}\left(M ; \tau_{0}, \tau_{1}\right)\right\} .
$$

By continuous dependence on the initial data, the set $\mathcal{U}\left(\tau_{0}, \tau_{1}\right)$ is open in $\mathbf{R}^{2}$. We now consider the application $\ell$ defined by

$$
\ell(\alpha, k ; \tau)=\left\{l_{1}(\alpha, k ; \tau), l_{2}(\alpha, k ; \tau)\right\} .
$$

By our choice of the initial data, $\ell$ is $C^{\infty}$ with respect to $\alpha, k$ and $\tau$. We next denote by $d\left(\ell, \mathcal{U}\left(\tau_{0}, \tau\right) ; 0\right)$ the topological degree of the mapping $\ell(\cdot, \cdot ; \tau)$ in the set $\mathcal{U}\left(\tau_{0}, \tau\right)$ at the value 0 . Since $\alpha=0, k=0$ is the only point of $\mathcal{U}\left(\tau_{0}, \tau_{1}\right)$ where $\ell\left(\alpha, k ; \tau_{0}\right)=0$, one then has that $d\left(\ell, \mathcal{U}\left(\tau_{0}, \tau_{0}\right) ; 0\right)=1$. The idea is then to prove that for every $\tau \geq \tau_{0}, \mathcal{U}\left(\tau_{0}, \tau\right) \neq \emptyset$ and $d\left(\ell, \mathcal{U}\left(\tau_{0}, \tau\right) ; 0\right)=1$. This is deduced from the continuity of $\ell$ and the fact that $\ell$ cannot vanish at the boundary of $\mathcal{U}\left(\tau_{0}, \tau\right)$, which follows from step two. This allows us to extend $\phi(\xi, \tau ; \alpha, k)$ as $\tau \longrightarrow \infty$, thus obtaining the global existence of $\phi$ in $\mathcal{A}\left(M ; \tau_{0},+\infty\right)$ and the required convergence to $\bar{\phi}$.

We next proceed to implement the plan just described.

5.2. Selecting the initial value away from the smoothed shocks. The purpose of this subsection is to determine how the coefficient $\alpha$ in the initial data has to be taken. As in Subsection 4.3, we rescale the variable $\xi$ near $x_{1}$ by setting

$$
\lambda=\left(\xi-x_{1}\right) e^{\tau / 2} .
$$

We then define

$$
\varphi(\lambda, \tau)=\phi\left(x_{1}+\lambda e^{-\tau / 2}, \tau\right)-\bar{\phi}\left(x_{1}+\lambda e^{-\tau / 2}\right)
$$

As we noticed in Section 4, $\varphi$ satisfies (4.22). Let us denote by $\zeta$ a smooth positive cut-off function of compact support contained in $(0,2 M)$, which is equal to one in $(\delta, M)$ for some small positive $\delta$. We set

$$
\psi(\lambda, \tau)=\left(\varphi(\lambda, \tau)-e^{-\tau} \Phi_{1}\left(x_{1}+\lambda e^{-\tau / 2}\right)\right) \zeta\left(x_{1}+\lambda e^{-\tau / 2}\right), \quad \text { for all } \lambda \in \mathbf{R} .
$$

As described in 5.1.2, at $\tau=\tau_{0}$ we pick the initial data $\phi_{0}$ such that

$$
\psi\left(\lambda, \tau_{0}\right)=\alpha \zeta\left(x_{1}+\lambda e^{-\tau_{0} / 2}\right),
$$

where $\alpha$ is a real constant. Observe that this choice means that the initial data $\phi_{0}$ satisfies

$$
\phi_{0}\left(\xi, \tau_{0}\right)=\bar{\phi}(\xi)+\phi_{1}(\xi)+\alpha
$$

for $\xi$ in $(\delta, M)$. We then prove

Lemma 5.1. Let $\varphi(\lambda, \tau)$ be the solution of (4.22) in the interval $\left[\tau_{0}, \tau_{1}\right]$ with initial data $\varphi\left(\lambda, \tau_{0}\right)$ given by (5.13). Suppose the constant $\alpha$ is such that

$$
\left\langle L_{0}, \psi\left(\lambda, \tau_{1}\right)\right\rangle=0,
$$

where $L_{0}$ is defined in $(4.27 b)$. Then

$$
\alpha=O\left(e^{-2 \tau_{0}}\right),
$$


and $\psi(\lambda, \tau)$ given in (5.12) is such that, for every positive constant $\Lambda$, there exists $\varepsilon=\varepsilon\left(\tau_{0}\right)$ with $\lim _{\tau_{0}} \longrightarrow \infty \varepsilon\left(\tau_{0}\right)=0$ such that

$$
|\psi(\lambda, \tau)| \leq \varepsilon e^{-3 \tau / 2} \quad \text { for all } \lambda \in(-\Lambda, \Lambda) \text { and all } \tau \in\left[\tau_{0}, \tau_{1}\right]
$$

Proof. In view of (4.22), it turns out that $\psi$ satisfies the following equation

$$
\psi_{\tau}=\psi_{\lambda \lambda}-\frac{3}{2} \lambda \psi_{\lambda}+g(\lambda, \tau) \quad \text { for all } \lambda \in \mathbf{R} \text { and } \tau \in\left[\tau_{0}, \tau_{1}\right],
$$

where

$$
\begin{aligned}
g(\lambda, \tau)= & J_{1}(\lambda, \tau)+\frac{\partial J_{2}}{\partial \lambda}(\lambda, \tau), \\
J_{1}(\lambda, \tau)= & +e^{-2 \tau} \zeta_{i}\left(\phi_{1 \xi \xi}+3 \phi_{1 \xi}\right)+e^{-3 \tau+\xi}\left(3 \phi_{1}+\phi_{1 \xi}\right) \zeta \\
& +2 e^{-2 \tau}+2 \bar{\psi} \psi-\bar{\psi}^{2} \zeta^{\prime} e^{\tau / 2} \\
& +\psi\left\{\frac{\lambda^{2}}{2} e^{-\tau}+\frac{\lambda}{2} e^{-\tau / 2}-2 e^{-\tau}+3 e^{-2 \tau+\xi}\right. \\
& \left.+e^{-\tau}\left(\phi_{1}+\phi_{1 \xi}\right)-2 e^{-2 \tau} e^{\lambda e^{-\tau / 2}}-2 \lambda o\left(e^{-\tau} e^{\tau / 2}\right)\right\} J_{2}(\lambda, \tau) \\
= & \left\{o\left(e^{-\tau}\right) e^{\tau / 2} \lambda^{2} \psi+e^{\tau / 2} \bar{\psi}^{2} \zeta+e^{-\tau / 2} \psi+\frac{1}{2} e^{-\tau / 2} \lambda^{2} \psi+2 e^{-3 \tau / 2} e^{r e^{-\tau / 2}} \psi\right\}
\end{aligned}
$$

and $\bar{\psi}$ is given by

$$
\bar{\psi}(\lambda, \tau)=\left(\varphi(\lambda, \tau)-e^{-\tau} \Phi_{1}\left(x_{1}+\lambda e^{-\tau / 2}\right)\right) .
$$

On the other hand, the operator

$$
A \psi=-\psi_{\lambda \lambda}+\frac{3}{2} \lambda \psi_{\lambda}
$$

is the generator of an analytical semigroup $S(\tau)$ on the space $L_{\omega}^{2}(\mathbf{R})$ defined in Section 4. In fact, if $G(x, t)$ is the heat kernel, $G(x, t)=(4 \pi t)^{-1 / 2} \exp \left(-\frac{|x|^{2}}{4 t}\right)$, and $u(x, t)=\left(G(t) * v_{0}\right)$, then $v(y, s)=u\left(e^{-3 s / 2} y, 1-e^{-3 s}\right)$ solves

$$
\frac{\partial v}{\partial s}-A v=0
$$

Moreover, since the first eigenvalue of $A$ is 0 , for every $s>0$ we have

$$
\|v(s)\| \leq\left\|v_{0}\right\| \text {. }
$$

By standard results, the solution $\psi$ of (5.17) can be written in the form

$$
\psi(\lambda, \tau)=S\left(\tau-\tau_{0}\right) \psi\left(\cdot, \tau_{0}\right)+\int_{\tau_{0}}^{\tau} S(\tau-s) g(\cdot, s) d s
$$

Due to the spectral properties of the operator $A$, we may represent $\psi$ in the following way

$$
\psi(\lambda, \tau)=\sum_{k=0}^{\infty} a_{k}(\tau) L_{k}(\lambda)
$$

Suppose now that the constant $\alpha$ is such that $\left\langle\psi\left(\lambda, \tau_{1}\right), L_{0}\right\rangle=0$, or equivalently

$$
\begin{gathered}
\int_{\mathbf{R}}\left(\phi\left(x_{1}+\lambda e^{-\tau_{1} / 2}, \tau_{1}\right)-\bar{\phi}\left(x_{1}+\lambda e^{-\tau_{1} / 2}, \tau_{1}\right)-e^{-\tau_{1}} \phi_{1}\left(x_{1}+\lambda e^{-\tau_{1} / 2}, \tau_{1}\right)\right) \\
\times e^{-\lambda^{2} / 4} \zeta\left(x_{1}+\lambda e^{-\tau_{1} / 2}, \tau_{1}\right) d \lambda=0 .
\end{gathered}
$$


On taking the scalar product with $L_{0}$ in (5.18) we obtain

$$
a_{0}\left(\tau_{0}\right)=-\int_{\tau_{0}}^{\tau_{1}}\left\langle S\left(\tau_{1}-s\right) g(\cdot, s), L_{0}\right\rangle d s
$$

We now claim that

$$
\int_{\tau_{0}}^{\tau_{1}}\left\langle S\left(\tau_{1}-s\right) g(\cdot, s), L_{0}\right\rangle d s=O\left(e^{-2 \tau_{0}}\right) .
$$

In order to show this, we observe that

$$
\begin{aligned}
& \left|\int_{\tau_{0}}^{\tau_{1}}\left\langle S\left(\tau_{1}-s\right) g(\cdot, s), L_{0}\right\rangle d s\right|=\mid \int_{\tau_{0}}^{\tau_{1}}\left\langle S\left(\tau_{1}-s\right)\left(J_{1}(\cdot, s)+\frac{\partial J_{2}}{\partial \lambda}(\cdot, s), L_{0}\right\rangle d s\right| \\
& \leq \int_{\tau_{0}}^{\tau_{1}}\left|\left\langle J_{1}(\cdot, s), S\left(\tau_{1}-s\right) L_{0}\right\rangle\right| d s+\int_{\tau_{0}}^{\tau_{1}}\left|\left\langle S\left(\tau_{1}-s\right) \frac{\partial J_{2}}{\partial \lambda}(\cdot, s), L_{0}\right\rangle\right| d s \\
& \leq \int_{\tau_{0}}^{\tau_{1}}|| J_{1}(\cdot, s)\left\|d s+\int_{\tau_{0}}^{\tau_{1}}\right\| \frac{\partial J_{2}}{\partial \lambda}(\cdot, s) \| d s \\
& \leq \int_{\tau_{0}}^{\tau_{1}}\left\|J_{1}(\cdot, s)\right\| d s+\int_{\tau_{0}}^{\tau_{1}} e^{-3 \frac{s-\tau_{0}}{4}}\left(\frac{3}{1-e^{-3\left(s-\tau_{0}\right)}}\right)^{1 / 2}\left\|J_{2}(\cdot, s)\right\| d s \equiv I_{1}+I_{2} .
\end{aligned}
$$

To estimate the $\|\cdot\|$ norm of each of these terms, we observe that by construction the function $\zeta\left(x_{1}+\lambda e^{-\tau / 2}\right)$ is different from zero only in the region where (5.5) holds. This implies that for all $\lambda \in \mathbf{R}$ and all $\tau \geq \tau_{0}$ we have $|\psi(\lambda, \tau)| \leq C(M) e^{-3 \tau / 2}$ and $|\bar{\psi} \zeta| \leq C(M) e^{-3 \tau / 2}$, for some positive constant $C(M)$ independent of $\tau_{0}$. This is actually sufficient to prove (5.21). This can be seen by noticing that the functions $J_{j}(\lambda, s)$ given in $(5.17 \mathrm{~b})$ are the sum of different terms. For the sake of brevity we do not estimate all of these. We analyze just one. Namely, we consider the following term appearing in $J_{2}$

$$
\int_{\tau_{0}}^{\tau_{1}} e^{-3 \frac{s-\tau_{0}}{4}}\left(\frac{3}{1-e^{-3\left(s-\tau_{0}\right)}}\right)^{1 / 2}\left\|o\left(e^{-\tau}\right) e^{\tau / 2} \lambda^{2} \psi\right\| d s
$$

By $(5.5)$,

$$
\begin{aligned}
& \left\|\lambda^{2} \psi(\cdot, s)\right\|^{2}=\int_{\mathbf{R}}\left|\lambda^{2} \psi(\lambda, s)\right|^{2} e^{-\lambda^{2} / 4} d \lambda \\
& \leq C(M) e^{-3 \tau / 2} \int_{\mathbf{R}}|\lambda|^{2}(1+|\lambda|)^{2} e^{-\lambda^{2} / 4} d \lambda,
\end{aligned}
$$

whence

$$
\begin{aligned}
& \int_{\tau_{0}}^{\tau_{1}} e^{-3 \frac{s-\tau_{0}}{4}}\left(\frac{3}{1-e^{-3\left(s-\tau_{0}\right)}}\right)^{1 / 2}\left\|o\left(e^{-\tau}\right) e^{\tau / 2} \lambda^{2} \psi\right\| d s \\
& \leq \int_{\tau_{0}}^{\tau_{1}} e^{-3 \frac{s-\tau_{0}}{4}}\left(\frac{3}{1-e^{-3\left(s-\tau_{0}\right)}}\right)^{1 / 2} e^{-s / 2} C(M) e^{-3 s / 2} d s \leq C(M) e^{-2 \tau_{0}}
\end{aligned}
$$

Similar estimates hold for all the remaining terms in $J_{1}$ and $J_{2}$, whereupon (5.21) follows. To proceed further and prove (5.16), we split (5.19) as follows

$$
\psi(\lambda, \tau)=a_{0}(\tau) L_{0}+R(\lambda, \tau) .
$$

The term $R(\cdot, \cdot)$ is the projection of $\psi$ on the set orthogonal to the first eigenspace of $A$ in $L_{\omega}^{2}(\mathbf{R})$. Therefore, it satisfies the equation

$$
R_{\tau}=A R+\bar{g}(\lambda, \tau)
$$


where $\bar{g}$ is the projection of $g$. Then, for every $\tau$ in $\left[\tau_{0}, \tau_{1}\right]$,

$$
R(\lambda, \tau)=S\left(\tau-\tau_{0}\right) R\left(\cdot, \tau_{0}\right)(\lambda)+\int_{\tau_{0}}^{\tau} S(\tau-s) \bar{g}(\cdot, s) d s \equiv R_{1}+R_{2} .
$$

Now, since the second eigenvalue of $A$ is $-3 / 2$, we deduce that

$$
\left\|R_{2}\right\| \leq \int_{\tau_{0}}^{\tau}\|S(\tau-s) \bar{g}(\cdot, s)\| d s \leq \int_{\tau_{0}}^{\tau} e^{-3(\tau-s) / 2}\left\|g_{i}(\cdot, s)\right\|_{w} d s
$$

Using the estimates on $\|g\|$ as before, we finally obtain

$$
\left\|R_{2}\right\| \leq C \int_{\tau_{0}}^{\tau} e^{-3(\tau-s) / 2} e^{-2 s} d s \leq C e^{-3 \tau / 2} e^{-\tau_{0} / 2} \text { for all } \tau \in\left[\tau_{0}, \tau_{1}\right]
$$

On the other hand,

$$
\begin{aligned}
\left\|R_{1}(\tau)\right\| & \leq e^{-3\left(\tau-\tau_{0}\right) / 2}\left\|R\left(\cdot, \tau_{0}\right)\right\| \leq e^{-3\left(\tau-\tau_{0}\right) / 2}\left\|\psi\left(\cdot, \tau_{0}\right)\right\| \\
& =C e^{-3\left(\tau-\tau_{0}\right) / 2} \alpha=C e^{-3\left(\tau-\tau_{0}\right) / 2} O\left(e^{-2 \tau_{0}}\right) \\
& \leq C e^{-3 \tau / 2} e^{-\tau_{0} / 2}
\end{aligned}
$$

This provides a bound for $R(\lambda, \tau)$ in $L_{\text {loc }}^{2}\left(\mathbf{R} \times\left[\tau_{0}, \tau_{1}\right]\right)$, which in turn gives an estimate in $L_{\mathrm{loc}}^{\infty}\left(\mathbf{R} \times\left(\tau_{0}, \tau_{1}\right]\right.$ by standard parabolic regularity. In particular, one has that

For any $\delta>0$ and any $\Lambda>0$ there exists $C>0$ such that

$$
|R(\lambda, \tau)| \leq C e^{-3 \tau / 2} e^{-\tau_{0} / 2}, \quad \text { for } \lambda \in(-\Lambda, \Lambda) \text { and } \tau \in\left[\tau_{0}+\delta, \tau_{1}\right],
$$

which yields (5.16) for $\tau \in\left[\tau_{0}+\delta, \tau_{1}\right]$. In order to prove (5.16) for $\tau$ near $\tau_{0}$, we use the available estimate on the initial data, as well as the continuous dependence of the solution on its initial value. More precisely, we have

$$
\left\|R\left(\cdot, \tau_{0}\right)\right\|_{\infty} \leq\left\|\psi\left(\cdot, \tau_{0}\right)\right\|_{\infty}=\left|\alpha\left(\tau_{0}\right)\right|\left\|\zeta\left(x_{1}+\cdot e^{-\tau_{0} / 2}\right)\right\|_{\infty} \leq C e^{-2 \tau_{0}} .
$$

On the other hand, by continuous dependence, for all positive constants $\Lambda>0$ and for all $\tau_{0}$ there exists $\delta_{1}>0$ such that for all $\tau \in\left[\tau_{0}, \tau_{0}+\delta_{1}\right)$ and every $\lambda \in(-\Lambda, \Lambda)$ we have

$$
\mid \psi(\lambda, \tau)-\psi\left(\lambda, \tau_{0} \mid \leq e^{-2 \tau_{0}}\right.
$$

Then, if we write

$$
\delta=\min \left(\delta_{1}, \tau_{0} / 6\right)
$$

we deduce that for all $\tau \in\left[\tau_{0}, \tau_{0}+\delta\right)$ and $\lambda \in(-\Lambda, \Lambda)$

$$
\begin{aligned}
|\psi(\lambda, \tau)| & \leq C e^{-2 \tau_{0}} \equiv e^{-3 \tau / 2} C e^{-2 \tau_{0}+3 \tau / 2} \\
& \leq e^{-3 \tau / 2} C e^{-2 \tau_{0}+3\left(\tau_{0}+\delta\right) / 2} \leq C e^{-3 \tau / 2} e^{-\tau_{0} / 4},
\end{aligned}
$$

where we have used (5.26) in the last inequality. Putting together (5.23) and (5.27), we obtain the result.

Lemma 5.1 shows that if $\phi_{0}$ satisfies (5.6) with constant $M$, and the initial data is suitably selected, the solution $\phi$ satisfies the estimate in (5.6), still with same constant $M$, in the region where $\xi-x_{1}=O\left(e^{-\tau / 2}\right)$ for $\tau \in\left[\tau_{0}, \tau_{1}\right]$. We now extend (5.16) up to regions where $\xi \geq \tau^{2} / 2 e^{-\tau}$. To this end we prove 
Lemma 5.2. Let $\phi$ as in the statement of Lemma 5.1. Then, for all $\tau \in\left[\tau_{0}, \tau_{1}\right]$ and for all $\xi$ such that $\frac{\tau^{2}}{2} e^{-\tau} \leq \xi \leq M$,

$$
\left|\phi(\xi, \tau)-\bar{\phi}(\xi)-e^{-\tau} \phi_{1}(\xi)\right| \leq \varepsilon e^{-\tau},
$$

where $\varepsilon=\varepsilon\left(\tau_{0}\right) \longrightarrow 0$ as $\tau_{0} \longrightarrow \infty$.

Proof. We shall proceed by means of a comparison argument. Let us consider the case where $\xi \in\left(0, x_{1}\right)$, the case where $\xi \in\left(x_{1}, M\right)$ being similar. To this end we define a function $H$ as follows

$$
\phi(\xi, \tau)-\bar{\phi}(\xi)-e^{-\tau} \phi_{1}(\xi)=H(\xi, \tau) .
$$

Then $H$ satisfies

$$
\begin{aligned}
H_{\tau} & =e^{-\tau} H_{\xi \xi}+(2 \bar{\phi}-1) H_{\xi}+(2 \bar{\phi}-1) H+2\left(H^{2}+H H_{\xi}\right) \\
& +e^{-\tau}\left\{H_{\xi}-2 H+2 \phi_{1}\left(H+H_{\xi}\right)\right\}+e^{-2 \tau}\left(\phi_{1 \xi \xi}+\phi_{1 \xi}-2 \phi_{1}\right) \\
& +e^{-2 \tau+\xi}\left(2 \bar{\phi}+2 e^{-\tau} \phi_{1}+3 H+H_{\xi}\right)
\end{aligned}
$$

for $\tau \in\left[\tau_{0}, \tau_{1}\right]$ and for $\xi \in \mathbf{R}$. We shall consider this equation on an interval $\left(2 M e^{-\tau}, x_{1}-R e^{-\tau / 2}\right)$. Observe that, by (5.6),

$$
\left|H\left(2 M e^{-\tau}, \tau\right)\right| \leq M e^{-\tau} \text { for all } \tau \in\left[\tau_{0}, \tau_{1}\right] .
$$

Using Lemma 5.1, we see that for every $R>0$ there exists $\varepsilon>0$ such that

$$
\begin{aligned}
& \left|H\left(x_{1}-R e^{-\tau / 2}, \tau\right)\right| \leq \varepsilon e^{-3 \tau / 2} \quad \text { for all } \tau \in\left[\tau_{0}, \tau_{1}\right], \\
& \text { and } \varepsilon \longrightarrow \infty \text { as } \tau_{0} \longrightarrow \infty .
\end{aligned}
$$

The function $H$ is then a solution of (5.30) on $\left(2 M e^{-\tau}, x_{1}-R e^{-\tau / 2}\right)$ with boundary conditions (5.31), (5.32). We now produce sub- and supersolutions of the boundary value problem $(5.30)-(5.32)$ on the interval $\left(0, x_{1}-R e^{-\tau / 2}\right)$. To this end, we first exhibit sub- and supersolutions $\underline{H}$ and $\bar{H}$ of the reduced problem

$$
\mathcal{L}(H) \equiv H_{\tau}-e^{-\tau} H_{\xi \xi}-(2 \bar{\phi}-1) H_{\xi}-(2 \bar{\phi}-1) H=0,
$$

with boundary conditions (5.32). Then, in a second step, we check that for the auxiliary function thus derived, the terms of the equation (5.30) which are not retained in (5.33) are of lower order when $\tau_{0} \gg 1$ and then $\underline{H}$ and $\bar{H}$ are respectivelly suband supersolutions of (5.30) if $\tau_{0}$ is large enough. We just describe the construction of the positive supersolution, since the negative subsolution is similarly arrived at.

By our choice of $\bar{\phi}$, we are thus led to consider the equation

$$
H_{\tau}=e^{-\tau} H_{\xi \xi}+\left(2 e^{-\xi}-1\right) H_{\xi}+\left(2 e^{-\xi}-1\right) H,
$$

on the interval $\left(2 M e^{-\tau}, x_{1}-R e^{-\tau / 2}\right)$ with boundary conditions

$$
\left|H\left(2 M e^{-\tau}, \tau\right)\right| \leq M e^{-\tau}, \quad\left|H\left(x_{1}-R e^{-\tau / 2}, \tau\right)\right| \leq \varepsilon e^{-3 \tau / 2} .
$$

The supersolution is obtained by setting

$$
\bar{H}(\xi, \tau)=H_{1}(\xi, \tau)+H_{2}(\xi, \tau),
$$

where $H_{1}(\xi, \tau)=2 \varepsilon e^{-\tau}\left(2 e^{-\xi}-1\right)+f_{1}(\xi, \tau), f_{1}(\xi, \tau)=e^{-3 \tau / 2} g(\xi), g$ is a solution of

$$
-\frac{3}{2} g-\left(2 e^{-\xi}-1\right)\left(g^{\prime}+g\right)=3, \quad g \sim-2 \quad \text { as } \xi \longrightarrow x_{1}^{-},
$$


and

$$
H_{2}(\xi, \tau)=2 M e^{\frac{1}{4} M} e^{-\tau} e^{-\frac{1}{8} \xi e^{\tau}} h(\xi),
$$

$h$ being a nonnegative cut-off function with support in the interval $(0,1 / 10)$, equal to 1 on $(0,1 / 20)$, and such that $0 \leq h \leq 1$ everywhere. In order to check that such a function is actually a supersolution of equation (5.34), a simple computation shows that

$$
\begin{aligned}
\mathcal{L}(\bar{H})= & \mathcal{L}\left(H_{1}\right)+\mathcal{L}\left(H_{2}\right) \\
= & e^{-3 \tau / 2}\left\{-\frac{3}{2} g-\left(2 e^{-\xi}-1\right)\left(g+g^{\prime}\right)-4 \varepsilon e^{-\xi} e^{-\tau / 2}-e^{-\tau}\left\|g^{\prime \prime}\right\|_{\infty}\right\} \\
& +M e^{\frac{1}{4} M} e^{-\frac{1}{8} \xi e^{\tau}}\left\{\delta h(\xi) e^{\tau}+\left(\frac{3}{2}-2 e^{-\xi}\right) h^{\prime}(\xi)+2 e^{-\tau} h^{\prime \prime}(\xi)\right\}
\end{aligned}
$$

where $\delta$ is a positive constant depending only on the choice of $h$. On the other hand, it is easily seen that

$$
g(\xi)=-\frac{3}{2} e^{-\xi}\left(2-e^{\xi}\right)^{2} \int_{0}^{\xi} \frac{e^{-2 \eta}}{\left(2-e^{\eta}\right)^{3}} d \eta
$$

whence

$$
e^{-\tau}\left|g^{\prime \prime}(\xi)\right| \leq C e^{-\tau} \log \left(x_{1}-\xi\right) \leq C \tau e^{-\tau}, \quad \text { for all } \xi \in\left(0, x_{1}-R e^{-\tau / 2}\right),
$$

for some positive constant $C$. Therefore, if $\tau_{0}$ is sufficiently large,

$$
\mathcal{L}(\bar{H}) \geq \frac{3}{2} e^{-3 \tau / 2}-2 M e^{\frac{1}{4} M} e^{-\frac{1}{80} e^{\tau}} \geq 2 e^{-3 \tau / 2} .
$$

Moreover, $H_{2}$ is nonnegative, and concerning $H_{1}$, for every $\xi \in\left(0, x_{1}-R e^{-\tau / 2}\right)$ we have

$$
H_{1}(\xi, \tau)=2 \varepsilon e^{-\tau}\left(2 e^{-\xi}-1\right)+e^{-3 \tau / 2} g(\xi) \geq e^{-3 \tau / 2}\left(R-\|g\|_{\infty}\right),
$$

which is positive if $R$ is sufficiently large. Then, for $R \gg 1, \bar{H}$ is nonnegative. As to the boundary values of $\bar{H}$, we have

$$
\begin{gathered}
\bar{H}\left(2 M e^{-\tau}, \tau\right) \geq 2 M e^{-\tau}, \\
\bar{H}\left(x_{1}-R e^{-\tau / 2}\right) \geq R \varepsilon e^{-3 \tau / 2}-2 e^{-3 \tau / 2}=e^{-3 \tau / 2}(\varepsilon R-2) .
\end{gathered}
$$

The function $\bar{H}$ is then a supersolution of (5.34) on $\left(2 M e^{-\tau}, x_{1}-R e^{-\tau / 2}\right)$ for $R$ large enough, and satisfies the boundary conditions (5.31) - (5.32).

We now claim that $\bar{H}$ is a supersolution of the whole equation (5.30). Indeed, all the terms neglected in (5.30) to obtain (5.34) are $O\left(e^{-2 \tau}\right)$ when evaluated for the function $H$. Therefore, if $\tau_{0}$ is sufficiently large,

$$
\mathcal{L}(\bar{H}) \geq 2 e^{-3 \tau / 2}
$$

on the interval $\left(2 M e^{-\tau}, x_{1}-R e^{-\tau / 2}\right)$. This concludes the proof of Lemma 5.2.

We next show

Lemma 5.3. Let $\phi$ be as in Lemma 5.2. Then, if $\tau_{0}$ is sufficiently large,

$$
|\phi(\xi, \tau)| \leq \varepsilon e^{-\tau} \quad \text { for all } \xi<-\frac{\tau^{2}}{2} e^{-\tau} \text { and } \tau \in\left[\tau_{0}, \tau_{1}\right]
$$

where $\varepsilon=\varepsilon\left(\tau_{0}\right) \longrightarrow 0$ as $\tau_{0} \longrightarrow \infty$. 
Proof. The proof will also proceed by means of a comparison argument. We consider again the function $H$ defined by (5.29), namely $\phi(\xi, \tau)-\bar{\phi}(\xi)-e^{-\tau} \phi_{1}(\xi)=H(\xi, \tau)$, which satisfies equation (5.30) on the region $\xi \leq-2 M e^{-\tau}, \tau \in\left[\tau_{0}, \tau_{1}\right]$. As in the proof of Lemma 5.2, it is easily checked that the following function is a supersolution

$$
\bar{H}=\left(1-e^{-\tau}\right)^{-\beta} e^{-a \frac{(\xi-\tau)^{2}}{\left(1-e^{-\tau}\right)}}+e^{\frac{\xi}{2} e^{\tau}} h(\xi),
$$

where $a$ and $\beta$ are such that $4 a<1$ and $2 a>\beta$, and where $h$ is a nonnegative cutoff function with support on $(-1 / 8,0)$, equal to one on $(-1 / 16,0)$ and satisfying $0 \leq h \leq 1$ everywhere. If we again define

$$
\mathcal{L}(\bar{H}) \equiv \bar{H}_{\tau}-e^{-\tau} \bar{H}_{\xi \xi}+\bar{H}_{\xi}+\bar{H},
$$

a routine calculation then gives

$$
\begin{aligned}
\mathcal{L}(\bar{H})= & \left(1-e^{-\tau}\right)^{-\beta-1} e^{-\tau} e^{-a \frac{(\xi-\tau)^{2}}{\left(1-e^{-\tau}\right)}}\left(2 a-\beta+\left(a-4 a^{2}\right) \frac{(\xi-\tau)^{2}}{1-e^{-\tau}}\right) \\
& +\frac{1}{2} e^{\frac{\xi}{2} e^{\tau}}\left(\frac{e^{\tau}}{2}\left(\frac{1}{2}+\xi\right) h(\xi)+h(\xi)-e^{-\tau} h^{\prime \prime}(\xi)\right) .
\end{aligned}
$$

It is then clear that for large $\tau_{0}, \mathcal{L}(\bar{H}) \geq 0$ for $\xi \leq-2 M e^{-\tau}$ and $\tau \in\left[\tau_{0}, \tau_{1}\right]$. The same argument as in the previous lemma shows that in fact $\bar{H}$ is a supersolution of the complete equation (5.30). Moreover,

$$
\bar{H}\left(-2 M e^{-\tau}, \tau\right) \geq e^{-M} .
$$

Now we want to apply the comparison principle to the functions $\bar{H}$ and $H$. The only difficulty is that we have to do it in the unbounded domain

$$
\mathcal{Q}=\left\{(\xi, \tau) ; \tau \in\left[\tau_{0}, \tau_{1}\right], \xi \in\left(-\infty,-2 M e^{-\tau}\right)\right\} .
$$

Let us then consider the sequence of functions $H_{n}$ defined as the unique solutions of equation (5.30) for $\tau \in\left[\tau_{0}, \tau_{1}\right], \xi \in(-n, 0)$ with initial data $H(\xi, 0)$ and with boundary conditions

$$
H_{n}(-n, \tau)=0, \quad H_{n}(0, \tau) \equiv H(0, \tau) .
$$

Using the uniqueness result in Theorem 2, as well as the supersolutions constructed in Section 3, it is straightforward to check that $H_{n} \longrightarrow H$ pointwise as $n \longrightarrow \infty$. On the other hand, by the maximum principle, $H_{n} \leq \bar{H}$ on the set

$$
\mathcal{Q}_{n}=\left\{(\xi, \tau) ; \tau \in\left[\tau_{0}, \tau_{1}\right], \xi \in\left(-n,-2 M e^{-\tau}\right)\right\} .
$$

Passing to the limit as $n \longrightarrow \infty$, we deduce that $H \leq \bar{H}$ for $\tau \in\left[\tau_{0}, \tau_{1}\right]$ and $\xi \leq$ $-2 M e^{-\tau}$. A similar construction gives a subsolution and the symmetric bound from below, whereupon Lemma 5.3 follows.

We also have

Lemma 5.4. For all $\xi>M$ and all $\tau \in\left[\tau_{0}, \tau_{1}\right]$,

$$
\phi(\xi, \tau) \leq\left\{\begin{array}{l}
M e^{-\xi} \quad \text { if } \xi \leq \tau-M, \\
M e^{2 \tau-3 \xi} \quad \text { if } \xi \geq \tau-M .
\end{array}\right.
$$


Proof. When the inverse of the change of variable defined in (4.4) is performed, we obtain the function

$$
x f_{0}(x)=\phi(\log x) .
$$

By our choice of the initial data $\phi_{0}$, we have, for some of the supersolutions $\bar{f}_{\alpha}$ of (1.1a) defined in (3.5), $f_{0}(x) \leq \bar{f}_{\alpha}(x)$. Then, the corresponding solution $f(x, t)$ of (1.1a) with initial data $f_{0}(x)$ satisfies

$$
f(x, t) \leq \bar{f}_{\alpha}(x)
$$

for all $t>0$ and all $x \in(0, \infty)$. Therefore a solution $\phi$ of (4.5) with initial data at $\tau=\tau_{0}$ equal to $\phi_{0}$ exists for all $\tau \geq \tau_{0}$ and is bounded from above by the function

$$
\bar{\phi}_{\alpha}(\xi, \tau)=e^{\xi} e^{-2 \tau} \bar{f}_{\alpha}\left(e^{-\tau} e^{\xi}\right) \leq\left\{\begin{array}{l}
\beta e^{-\xi} \text { if } \xi \leq \tau, \\
\beta e^{2 \tau-3 \xi} \quad \text { if } \xi \geq \tau .
\end{array}\right.
$$

Lemma 5.4 then follows by taking $M>\beta$.

5.3. The region near the smoothed shocks. In the previous subsection we obtained an estimate of $\phi$ in the regions $|\xi|>\tau^{2} e^{-\tau} / 2, \xi>x_{1}$ and $\xi<-\tau^{2} e^{-\tau} / 2$. We shall now bound $\phi$ in the region $|\xi| \leq \tau^{2} e^{-\tau} / 2$. For that purpose we consider the auxiliary function

$$
\Psi(z, \sigma)=\phi(\xi, \tau), \quad z=(\xi-\lambda(\tau)) e^{\tau}, \quad \sigma=e^{\tau} .
$$

Observe that estimating $\Psi$ in the region where $|z| \leq \tau^{2}$ will provide, just by changing variables, an estimate on $\phi$ in the region $|\xi-\lambda(\tau)| \leq \tau^{2} e^{-\tau}$. Since by assumption $\phi \in \mathcal{A}\left(M ; \tau_{0}, \tau_{1}\right)$, we may suppose that $|\lambda(\tau)| \leq M e^{-\tau}$. Therefore we will have a suitable bound on $\phi$ in the region $|\xi| \leq\left(\tau^{2}-M\right) e^{-\tau}$, and thus in particular in the region $|\xi| \leq \tau^{2} e^{-\tau} / 2$ as required.

In order to obtain the desired bounds, the following technical construction will be used. Let $\bar{\tau}$ be any fixed value in the interval $\left(\tau_{0}, \tau_{1}\right)$, and let us define the new variables

$$
\eta=(\xi-\lambda(\bar{\tau})) e^{\tau} \quad \text { and } \quad \Phi(\eta, \tau)=\phi(\xi, \tau),
$$

for $\tau$ such that $\bar{\tau}<\tau<\tau_{1}$. The function $\Phi$ satisfies the equation

$$
\begin{aligned}
e^{-\tau} \Phi_{\tau}= & \Phi_{\eta \eta}+(2 \Phi-1) \phi_{\eta}+e^{-\tau}\left((2 \Phi-1) \Phi-\eta \Phi_{\eta}+\Phi_{\eta}\right) \\
& +2 e^{-2 \tau} \Phi+e^{-3 \tau} e^{\lambda(\bar{\tau})+\eta e^{-\tau}}\left(3 \Phi+e^{\tau} \Phi_{\eta}\right)
\end{aligned}
$$

for $\xi \in \mathbf{R}, \tau \geq \tau_{0}$. We want to estimate $\Phi$ in this new variable, in a region around the smoothed discontinuity point $\xi_{0}=0$. To this end, equation (5.39) is to be complemented with suitable boundary conditions. First of all, by our previous choice of $\bar{\phi}$ we have

$$
\phi_{1}(\xi)=\left\{\begin{array}{l}
2 e^{-\xi}+C_{0}\left(1-2 e^{-\xi}\right) \quad \text { if } \xi>0, \\
C_{2} \quad \text { if } \xi<0,
\end{array}\right.
$$

for some constants $C_{0}$ and $C_{2}$. We have just seen that for $|\xi| \geq \tau^{2} e^{-\tau} / 2$ and $\tau \in\left(\tau_{0}, \tau_{1}\right)$ we have

$$
\left|\phi(\xi, \tau)-\bar{\phi}(\xi)-e^{-\tau} \phi_{1}(\xi)\right| \leq \varepsilon e^{-\tau},
$$


where $\varepsilon=\varepsilon\left(\tau_{0}\right)$ is given in Lemma 5.2. Therefore, for every $\bar{\tau} \in\left(\tau_{0}, \tau_{1}\right)$

$$
\begin{aligned}
\left|\phi(\xi, \tau)-\bar{\phi}(\xi)-e^{-\bar{\tau}} \phi_{1}(\xi)\right| & \leq \varepsilon e^{-\tau}+\left|\phi_{1}(\xi)\right| e^{-\tau}\left|1-e^{\tau-\bar{\tau}}\right| \\
& \leq \varepsilon e^{-\tau}+\left\|\phi_{1}\right\|_{\infty} e^{-\tau}\left|1-e^{\tau-\bar{\tau}}\right| .
\end{aligned}
$$

Hence,

$$
\left|\phi(\xi, \tau)-\bar{\phi}(\xi)-e^{-\bar{\tau}} \phi_{1}(\xi)\right| \leq 2 \varepsilon e^{-\tau},
$$

for $\bar{\tau} \in\left(\tau_{0}, \tau_{1}\right)$ and $\tau \in\left(\bar{\tau}, \tau_{1}\right)$ such that

$$
\left\|\phi_{1}\right\|_{\infty}\left|e^{\tau-\bar{\tau}}-1\right| \leq \varepsilon
$$

On the other hand, since we assume that $\phi \in \mathcal{A}\left(M ; \tau_{0}, \tau_{1}\right)$, we have by (5.1) that $|\lambda(\bar{\tau})| \leq M e^{-\bar{\tau}}$. Therefore, for $\tau>\bar{\tau}$ satisfying

$$
e^{\tau-\bar{\tau}} \leq \frac{M}{2} \tau^{2}
$$

we certainly have that if $|\eta| \geq \tau^{2}$ then $|\xi| \geq \tau^{2} e^{-\tau} / 2$. Since

$$
\bar{\phi}(\xi)=1-\xi+O\left(\xi^{2}\right)=1-\left(\lambda(\bar{\tau})+\eta e^{-\tau}\right)+O\left(\xi^{2}\right)
$$

for $\xi \geq \tau^{2} e^{-\tau} / 2$, then

$$
\bar{\phi}(\xi)=1-\left(\lambda(\bar{\tau})+\eta e^{-\tau}\right)+O\left(\tau^{4} e^{-2 \tau}\right),
$$

whereas $\bar{\phi}(\xi)=0$ if $\xi \leq-\tau^{2} e^{-\tau} / 2$. On the other hand, $\phi_{1}(\xi)=C_{1}+O\left(\tau^{2} e^{-\tau}\right)$ when $\xi \geq \tau^{2} e^{-\tau} / 2$, and $\phi(\xi)=C$ if $\xi \leq-\tau^{2} e^{-\tau} / 2$. Therefore, for any $\tau>\bar{\tau}$ such that (5.40) and (5.41) hold, $\Phi$ satisfies

$$
\begin{gathered}
\left|\Phi(\eta, \tau)-1+\left(\lambda(\bar{\tau})+\eta e^{-\bar{\tau}}\right)-C_{1} e^{-\bar{\tau}}\right| \leq \varepsilon e^{-\tau} \quad \text { for } \quad \eta \geq \tau^{2}, \\
\left|\Phi(\eta, \tau)-C_{2} e^{-\bar{\tau}}\right| \leq \varepsilon e^{-\tau} \quad \text { for } \quad \eta \leq-\tau^{2} .
\end{gathered}
$$

In the interior region where $\eta \in\left(-\tau^{2}, \tau^{2}\right)$, so far we have only an estimate arising from the fact that function $\phi$ belongs to $\mathcal{A}\left(M ; \tau_{0}, \tau_{1}\right)$, namely $0 \leq \Phi(\eta, \tau) \leq M$. However, we can easily obtain estimates on the spatial derivative $\Phi_{\eta}$ in the following way. Set

$$
\Phi(\eta, \tau)=\psi\left(\eta, e^{\tau}\right),
$$

and write $\sigma=e^{\tau}, \sigma_{0}=e^{\tau_{0}}$ and $\sigma_{1}=e^{\tau_{1}}$. Then $\psi$ satisfies

$$
\begin{aligned}
\psi_{\sigma}= & \psi_{\eta \eta}+(2 \psi-1) \psi_{\eta}+e^{-\tau}\left((2 \psi-1) \psi-\eta \psi_{\eta}+\psi_{\eta}\right) \\
& +2 e^{-2 \tau} \psi+e^{-3 \tau} e^{\lambda(\bar{\tau})+\eta e^{-\tau}}\left(3 \psi+e^{\tau} \psi_{\eta}\right) .
\end{aligned}
$$

Moreover, $0 \leq \psi \leq M$ on the interval $\left[-\tau^{2}, \tau^{2}\right]$. Therefore, by classical parabolic regularity we deduce that for every $\rho>1$ there exists $C_{\rho}>0$ such that $\left|\psi_{\eta}\right| \leq C M$ on $\left(-\tau^{2}, \tau^{2}\right)$ whenever $\sigma_{1}>\sigma>\rho \sigma_{0}$. We can then rewrite equation (5.43b) in the following way

$$
\psi_{\sigma}=\psi_{\eta \eta}+(2 \psi-1) \psi_{\eta}-\sigma^{-1} \eta \psi_{\eta}+O\left(\frac{C_{M}}{\sigma}\right) .
$$

On the other hand, conditions (5.40) and (5.41) now give

$$
|| \phi_{1} \|_{\infty}|s-\bar{s}| \leq \varepsilon \bar{s}, \quad \frac{s}{\bar{s}} \leq \frac{M}{2} \log ^{2} s
$$


We then deduce from (5.42) that, for every $s$ and $\bar{s}$ in $\left(\sigma_{0}, \sigma_{1}\right)$ such that $s>$ $\bar{s}$ satisfying (5.45), and for all $\sigma \in(\bar{s}, s)$, the function $\psi$ satisfies the following boundary conditions

$$
\begin{gathered}
\left|\psi(\eta, \sigma)-1+\left(s(\log \bar{s})+\frac{\eta}{\bar{s}}\right)-\frac{C_{1}}{\bar{s}}\right| \leq 2 \frac{\varepsilon}{s} \quad \text { for } \quad \eta=\log ^{2} s, \\
\left|\psi(\eta, s)-\frac{C_{2}}{\bar{s}}\right| \leq 2 \frac{\varepsilon}{s} \quad \text { for } \quad \eta=-\log ^{2} s .
\end{gathered}
$$

Returning to the function $\Psi(z, \sigma)$ in (5.37), we have that, for all $\xi \in \mathbf{R}$ and $\tau, \bar{\tau}$ as before,

$$
\begin{aligned}
|\Psi(z, \sigma)-\psi(\eta, \sigma)| & =\left|\phi\left((\xi-\lambda(\tau)) e^{\tau}, \sigma\right)-\phi\left((\xi-\lambda(\bar{\tau})) e^{\tau}, \sigma\right)\right| \\
& \leq C(M)|\lambda(\tau)-\lambda(\bar{\tau})| e^{\tau} \leq C(M)(\tau-\bar{\tau}) .
\end{aligned}
$$

Therefore, if

$$
\tau-\bar{\tau} \leq \frac{\varepsilon}{10 C(M)}
$$

we deduce that

$$
|\Psi(z, \sigma)-\psi(\eta, \sigma)| \leq \frac{\varepsilon}{10} .
$$

In order to proceed further with our comparison arguments, we need an estimate on $\psi(\eta, \sigma)$ at $\eta=0$ (which is just the function $\phi$ near the point $\lambda(\bar{\tau})$ ). By definition, we know that for each $\tau \in\left[\tau_{0}, \tau_{1}\right]$ there is a single point, namely $\xi=\lambda(\tau)$, where $\phi(\lambda(\tau), \tau)=1 / 2$ and $\phi_{\xi}(\lambda(\tau), \tau)>0$. In the $\eta$ variable we then have that $\Phi(\eta, \tau)=$ $1 / 2$ at $\eta=\Lambda(\tau)$, where $\Lambda(\tau)=(\lambda(\tau)-\lambda(\bar{\tau})) e^{\tau}$. This value is unique, and $\Lambda(\bar{\tau})=$ 0 . Moreover, by hypothesis (see condition (5.5) of the definition of $\mathcal{A}\left(M ; \tau_{0}, \tau_{1}\right)$ ), $|d \lambda / d \tau| \leq M e^{-\tau}$ for every $\tau \in\left(\tau_{0}, \tau_{1}\right)$. Therefore, using the fact that

$$
\frac{d \Lambda}{d \tau}=\frac{d \lambda}{d \tau} e^{\tau}+e^{\tau}(\lambda(\tau)-\lambda(\bar{\tau}))
$$

we see that

$$
\left|\frac{d \Lambda}{d \sigma}\right| \equiv\left|\frac{d \Lambda}{d \tau} \cdot \frac{d \tau}{d \sigma}\right| \leq\left(M+M\left|1-e^{\tau-\bar{\tau}}\right|\right) \sigma^{-1} \leq\left(1+\frac{\varepsilon}{\left\|\phi_{1}\right\|_{\infty}}\right) \frac{M}{\sigma} .
$$

By the mean value theorem we deduce at once that

$$
|\Lambda(\sigma)| \leq\left(1+\frac{\varepsilon}{\left\|\phi_{1}\right\|_{\infty}}\right) \frac{M|\sigma-\bar{s}|}{\bar{s}} \text { for all } \sigma \geq \bar{s}
$$

and

$$
\left|\psi(0, \sigma)-\frac{1}{2}\right| \leq\left(1+\frac{\varepsilon}{\left\|\phi_{1}\right\|_{\infty}}\right) \frac{C(M) M|\sigma-\bar{s}|}{\bar{s}} .
$$

Therefore, if $s, \bar{s}$ satisfy (5.45), and

$$
\left\{\begin{array}{l}
|s-\bar{s}|<C_{M} \varepsilon \bar{s}, \\
C_{M}=\min \left(\frac{\left\|\phi_{1}\right\|_{\infty}}{\left(\left\|\phi_{1}\right\|_{\infty}+\varepsilon\right) 10 C(M)}, \frac{1}{\left\|\phi_{1}\right\|_{\infty}}\right),
\end{array}\right.
$$

we have

$$
\left|\psi(0, \sigma)-\frac{1}{2}\right| \leq \varepsilon \quad \text { for all } \sigma \in(\bar{s}, s)
$$

We now proceed to improve the estimates on $\Psi$ in the region $|z| \leq \log ^{2} s$. We begin with the following lemma. 
Lemma 5.5. Let $\Psi$ be the function defined above. Then for every $s \in\left(\sigma_{0}, \sigma_{1}\right)$ such that $s \geq \sigma_{0}+2 \log ^{2} \sigma_{0}$,

$$
\Psi(z, s) \leq 1+\varepsilon \quad \text { for any } z \in\left(0, \log ^{2} s\right)
$$

and

$$
\Psi(z, s) \geq-1-\varepsilon \quad \text { for any } z \in\left(-\log ^{2} s, 0\right) .
$$

Proof. Let us fix an arbitrary $s$ satisfying the hypothesis of the lemma and define $\bar{s} \geq \sigma_{0}$ as follows

$$
\bar{s}+2 \log ^{2} \bar{s}=s .
$$

A routine calculation shows that $s$ and $\bar{s}$ satisfy conditions (5.45) and (5.49) if $\sigma_{0}$ is sufficiently large. We are going to produce a supersolution of $(5.44),(5.46)$ and (5.50) on the interval $\left(0, \log ^{2} s\right)$. A similar construction gives a subsolution of (5.44), (5.47) and (5.50) on $\left(-\log ^{2} s, 0\right)$, and these two auxiliary functions will provide the estimate in the lemma. We look for a supersolution of the form $\bar{\psi}(\eta, \sigma)=$ $K_{M} \sigma^{2} / \bar{s}+g(\eta, \sigma)$, for $\sigma \in[\bar{s}, s]$, where $K_{M}$ is a positive constant satisfying

$$
K_{M}=O\left(C_{M} \frac{\log ^{2} s}{\sigma}\right) \text { for all } \sigma \in[\bar{s}, s] .
$$

Observe that $K_{M}$ can be selected arbitrarily small by taking $\sigma_{0}$ (or equivalently $\tau_{0}$ ) large enough. The function $g$ has to satisfy

$$
g_{\sigma} \geq g_{\eta \eta}+\left(2 K_{M} \frac{\sigma^{2}}{\bar{s}}+2 g-1\right) g_{\eta} .
$$

Since $2 K_{M} \sigma^{2} / \bar{s}>0$, it is enough for $g$ to be such that $g_{\eta}<0$ and

$$
g_{\sigma}=g_{\eta \eta}+(2 g-1) g_{\eta} .
$$

Let us define $g(\eta, \sigma)=\varphi(\eta-\sigma-\gamma, \sigma)$, where $\gamma$ is a sufficiently large positive constant, to be fixed later, and $\eta-\sigma=r$. Then $\varphi$ is required to satisfy Burgers equation

$$
\varphi_{\sigma}=\varphi_{r r}+2 \varphi \varphi_{r}
$$

As it is well known, this can be solved explicitly by means of the Cole-Hopf transformation $\varphi=\frac{W_{r}}{W}$, where $W$ now solves the heat equation $W_{\sigma}-W_{r r}=0$. In order to obtain our supersolution, let us take as initial value for $\varphi$ the function

$$
\varphi_{0}(r)= \begin{cases}\bar{M} \quad \text { if } \quad r<0, \\ 1+\varepsilon^{\prime} \quad \text { if } \quad r>0,\end{cases}
$$

with $\bar{M}>M$ and $\varepsilon^{\prime}=\varepsilon / 3$. The initial value for $W$ is then

$$
W_{0}(r)= \begin{cases}e^{\bar{M} r} & \text { if } \quad r<0, \\ e^{\left(1+\varepsilon^{\prime}\right) r} & \text { if } \quad r>0 .\end{cases}
$$

The function $W(r, \sigma)$ is given explicitly by the formula

$$
W(r, \sigma)=(4 \pi \sigma)^{-\frac{1}{2}} \int_{\mathbf{R}} e^{-\frac{|r-z|^{2}}{4 \sigma}} W_{0}(z) d z .
$$

In order to make explicit the behaviour of the function $\varphi$ in the region where $r+\sigma \in\left(0, \log ^{2} s\right)$, we perform the following change of variables

$$
W(r, \sigma)=e^{\left(1+\varepsilon^{\prime}\right) r} G(r, \sigma),
$$


where $G$ solves

$$
G_{\sigma}=G_{r r}+2\left(1+\varepsilon^{\prime}\right) G_{r}+\left(1+\varepsilon^{\prime}\right)^{2} G
$$

Therefore we can write $G$ as

$$
G(r, \sigma)=e^{\left(1+\varepsilon^{\prime}\right)^{2} \sigma} H\left(r+2\left(1+\varepsilon^{\prime}\right) \sigma, \sigma\right),
$$

with

$$
H_{0}(z)=\left\{\begin{array}{cc}
e^{\left(\bar{M}-\left(1+\varepsilon^{\prime}\right)\right) z} & \text { if } \quad z<0 \\
1 & \text { if } \quad z>0
\end{array}\right.
$$

Hence

$$
W(r, \sigma)=e^{\left(1+\varepsilon^{\prime}\right)^{2} s} e^{\left(1+\varepsilon^{\prime}\right) r} H\left(r+2\left(1+\varepsilon^{\prime}\right) \sigma, \sigma\right),
$$

and

$$
\varphi \equiv \frac{W_{r}}{W}=\left(1+\varepsilon^{\prime}\right)+\frac{H_{z}\left(r+2\left(1+\varepsilon^{\prime}\right) \sigma, \sigma\right)}{H\left(r+2\left(1+\varepsilon^{\prime}\right) \sigma, \sigma\right)} .
$$

Observe that $\varphi$ is a monotone increasing diffusive wave which propagates with speed $-2\left(1+\varepsilon^{\prime}\right)$ and connects the value $\bar{M}$ at $-\infty$ to the value $1+\varepsilon^{\prime}$ at $+\infty$.

The function $g$ is then given by

$$
g(\eta, \sigma)=\varphi(\eta-\sigma-\gamma, \sigma)=\left(1+\varepsilon^{\prime}\right)+\frac{H_{z}\left(\eta+2\left(1+\varepsilon^{\prime}\right) \sigma-\sigma-\gamma, \sigma\right)}{H\left(\eta+2\left(1+\varepsilon^{\prime}\right) \sigma-\sigma-\gamma, \sigma\right)},
$$

and $g$ is thus a diffusive travelling wave with speed $-1-2 \varepsilon$. We now choose $\gamma$ large enough so as to have

$$
g(\eta, \bar{s})>M \quad \text { for } \quad \eta \in\left(0, \log ^{2} s\right) .
$$

By construction $g \geq\left(1+\varepsilon^{\prime}\right)$, whereas by $(5.47)-(5.50)$

$$
\psi(0, \sigma) \leq 1+\varepsilon^{\prime}, \quad \psi\left(\log ^{2} s, \sigma\right) \leq 1+\varepsilon^{\prime} .
$$

We then deduce by comparison that

$$
\bar{\psi}(\eta, \sigma) \geq \psi(\eta, \sigma)
$$

for $\eta \in\left(0, \log ^{2} s\right)$ and for $\sigma \in(\bar{s}, s]$. We can then select the constant $\gamma$ so that

$$
\bar{\psi}(\eta, \sigma) \leq\left(1+2 \varepsilon^{\prime}\right) \quad \text { on } \quad\left(0, \log ^{2} s\right) \quad \text { for all } \sigma \geq \bar{s}+\frac{\log ^{2} s}{1+2 \varepsilon^{\prime}} .
$$

Finally, we observe that if $\tau_{0}$ is large enough,

$$
2 \log ^{2} \bar{s}>\frac{\log ^{2} s}{1+2 \varepsilon} \equiv \frac{\log ^{2} s}{1+2 \varepsilon / 3},
$$

and thus

$$
\bar{\psi}(\eta, s) \leq\left(1+\frac{2 \varepsilon}{3}\right) \quad \text { on } \quad\left(0, \log ^{2} s\right) .
$$

Lemma 5.4 follows now by using (5.48).

We now give a lower bound on the function $\Psi$ 
Lemma 5.6. Let $\Psi$ be the function defined in (5.37). Then, for all $s>\sigma_{0}+$ $\frac{1}{\pi \varepsilon^{2}} \log ^{4} \sigma_{0}$,

$$
\Psi(z, s) \geq \frac{1}{2}-2 \varepsilon \quad \text { for all } \eta \in\left(0, \log ^{2} s\right)
$$

and

$$
\Psi(z, s) \leq-\frac{1}{2}+2 \varepsilon \quad \text { for all } \eta \in\left(-\log ^{2} s, 0\right) .
$$

Proof. For every $s$ satisfying the hypothesis of the lemma let $\bar{s} \in\left[\sigma_{0}, s\right]$ be defined by

$$
\bar{s}+\frac{1}{\pi \varepsilon^{2}} \log ^{4} \bar{s}=s .
$$

As before, if $\tau_{0}$ is sufficiently large, $s$ and $\bar{s}$ satisfy (5.46) and (5.49). Therefore we have the estimates

$$
\begin{gathered}
\psi(0, \sigma) \geq \frac{1}{2}-\varepsilon, \\
\psi\left(\tau^{2}, \sigma\right) \geq 1-\varepsilon,
\end{gathered}
$$

for all $\sigma \in[\bar{s}, s]$. We now construct in detail a subsolution of $(5.44)$ on $\left(0, \log ^{2} s\right)$; a similar construction gives a supersolution on $\left(-\log ^{2}(\bar{s}), 0\right)$. We look for a function of the form

$$
\underline{\psi}(\eta, \sigma)=-K_{M} \log \frac{\sigma}{\bar{s}}+h(\eta, \sigma)
$$

for $\sigma \in[\bar{s}, s]$, where $K_{M}$ is a positive constant such that

$$
K_{M}=O\left(C_{M}\right) .
$$

To this end we now need $h$ to satisfy

$$
h_{\sigma} \leq h_{\eta \eta}+\left(2 h-1-2 K_{M} \log \frac{\sigma}{\bar{s}}-\sigma^{-1} \eta\right) h_{\eta} .
$$

By selecting $h$ monotone increasing, we will have

$$
K_{M} \log \left(\frac{\sigma}{\bar{s}}\right) h_{\eta}>0
$$

Moreover, if $\tau_{0}$ is large enough, $s$ and $\bar{s}$ satisfy

$$
\left|2 K_{M} \log \frac{\sigma}{\bar{s}}\right|+\left|\sigma^{-1} \log ^{2} s\right|<\varepsilon / 4,
$$

and it will be enough for $h$ to be such that

$$
h_{\sigma} \leq h_{\eta \eta}+(2 h-(1+\varepsilon)) h_{\eta} .
$$

Let $\underline{h}$ be the solution of

$$
\left\{\begin{array}{l}
\underline{h}_{\sigma}=\underline{h}_{\eta \eta} \text { for } \eta \geq 0, \sigma \geq \bar{s}, \\
\underline{h}(0, \sigma)=\frac{1}{2}-\varepsilon \quad \text { for } \sigma \geq \bar{s} \\
\underline{h}(\eta, \bar{s})=0 \text { for } \eta \geq 0 .
\end{array}\right.
$$

This function is explicitly given by

$$
\underline{h}(\eta, \sigma)=\left(\frac{1}{2}-\varepsilon\right)\left(1-\frac{2}{\sqrt{\pi}} \int_{0}^{\frac{\eta}{2 \sqrt{\sigma-s}}} e^{-\xi^{2} d \xi}\right) .
$$


It is readily seen that for every $\sigma \geq \bar{s}, \underline{h}(\cdot, \sigma)$ is a monotone decreasing function of $\eta$. This implies that $\underline{h} \leq 1 / 2-\varepsilon$ for all $\eta \geq 0$, and therefore

$$
\underline{h}_{\sigma} \leq \underline{h}_{\eta \eta}+(2 \underline{h}-(1+\varepsilon)) \underline{h}_{\eta}
$$

on $\left(0, \tau^{2}\right)$. This shows that

$$
\psi(\eta, \sigma) \geq \underline{h}(\eta, \sigma)-K_{M} \log \frac{\sigma}{\bar{s}}
$$

for $\eta \in\left(0, \tau^{2}\right)$ and $\sigma \in[\bar{s}, s]$. On the other hand, a direct computation shows that if

$$
\tau^{4} \leq \frac{\varepsilon^{2} \pi}{(1-2 \varepsilon)^{2}}(\sigma-\bar{s})
$$

then

$$
\underline{h}(\eta, \sigma) \geq\left(\frac{1}{2}-\varepsilon\right)\left(1-\frac{2 \varepsilon}{(1-2 \varepsilon)}\right)=\frac{1}{2}-\frac{3 \varepsilon}{2} .
$$

Observe now that if $\tau_{0}$ is sufficiently large, then

and therefore

$$
\frac{\pi \varepsilon^{2}}{(1-2 \varepsilon)^{2}}(s-\bar{s})=\frac{\bar{\tau}^{4}}{(1-2 \varepsilon)^{2}} \geq \tau^{4}
$$

$$
\underline{h}(\eta, s) \geq \frac{1}{2}-\frac{3 \varepsilon}{2}
$$

Using (5.51), we obtain

$$
\psi(\eta, s) \geq \underline{h}(\eta, s)-K_{M} \log \frac{s}{\bar{s}} \geq \frac{1}{2}-\frac{7 \varepsilon}{4} \quad \text { for all } \eta \in\left(0, \tau^{2}\right) .
$$

And finally the lemma follows by using (5.48c).

We improve this lower estimate in the next result.

Lemma 5.7. Let $\Psi$ be as in the previous lemma. Then there is a positive constant $R \equiv R(\varepsilon)$ such that

$$
\Psi(z, s) \geq 1-2 \varepsilon \quad \text { for any } z \in\left(R, \log ^{2} s\right)
$$

and

$$
\Psi(z, s) \leq-1+2 \varepsilon \quad \text { for any } z \in\left(-\log ^{2} s,-R\right),
$$

for every $s \in\left(\sigma_{0}, \sigma_{1}\right)$ satisfying

$$
s \geq \sigma_{0}+\frac{2}{\pi \varepsilon^{2}} \log ^{4} \sigma_{0}+\frac{2 \log ^{2} \sigma_{0}}{1+8 \varepsilon} .
$$

Proof. Fix any $s$ satisfying the hypothesis in the lemma, and let $\bar{s}>\frac{2}{\pi \varepsilon^{2}} \log ^{4} \sigma_{0}$ be such that

$$
\bar{s}+\frac{2 \log ^{2} \bar{s}}{1+8 \varepsilon}=s .
$$

As in the proof of our previous result, if $\tau_{0}$ is sufficiently large, $\bar{s}$ and $s$ satisfy conditions (5.46) and (5.49). By (5.42) and (5.50) the function $\psi$ then satisfies the following boundary conditions on $\left(0, \tau^{2}\right)$

$$
\psi(0, \sigma) \geq \frac{1}{2}-\varepsilon
$$


and

$$
\psi\left(\tau^{2}, \sigma\right) \geq 1-\varepsilon
$$

for all $\sigma \in[\bar{s}, s]$. On the other hand, by Lemma 5.5 we have

$$
\psi(\eta, \sigma) \geq \frac{1}{2}-2 \varepsilon \text { for } \sigma \in[\bar{s}, s] \eta \in\left(0, \tau^{2}\right) .
$$

We now construct a subsolution of (5.44) on $\left(0, \log ^{2} s\right)$. A similar construction gives a supersolution on $\left(-\log ^{2}(\bar{s}), 0\right)$. As before, we shall look for a function of the form

$$
\underline{\psi}(\eta, \sigma)=-K_{M} \log \frac{\sigma}{\bar{s}}+h(\eta, \sigma)
$$

with $K_{M}$ a positive constant such that $-K_{M}=O\left(C_{M}\right)$. As before we need $h$ to satisfy

$$
h_{\sigma} \leq h_{\eta \eta}+\left(2 h-1-2 K_{M} \log \frac{\sigma}{\bar{s}}-\sigma^{-1} \eta\right) h_{\eta} .
$$

By selecting $h$ monotone increasing we will have

$$
K_{M} \log \left(\frac{\sigma}{\bar{s}}\right) h_{\eta}>0
$$

Moreover, if $\tau_{0}$ is large enough, then $s$ and $\bar{s}$ obviously satisfy (5.51), and it will be enough for $h$ to satisfy

$$
h_{\sigma}=h_{\eta \eta}+(2 h-(1+\varepsilon)) h_{\eta} .
$$

We shall look for travelling wave solutions $h(\eta, \sigma)=G(\eta-c(\sigma-\bar{s})-\gamma)$ with $c<0$, $\gamma$ large enough, and $G$ such that

$$
-c G^{\prime}=G^{\prime \prime}+(2 G-(1+\varepsilon)) G^{\prime} .
$$

The general solution of (5.53) can be written as

$$
G(\eta)=\frac{\alpha \theta+\beta e^{(\beta-\alpha) \eta}}{\theta+e^{(\beta-\alpha) \eta}}
$$

where $\theta$ is an arbitrary constant and $\alpha$ and $\beta$ are such that $\alpha+\beta=1+\varepsilon-c$. We first require that

$$
\beta=1-\varepsilon \quad \text { and } \quad \alpha=\frac{1}{2}-4 \varepsilon
$$

Then, necessarily,

$$
c=-\frac{1}{2}-4 \varepsilon
$$

With this choice of parameters, the solution $G$ satisfies

$$
\lim _{\eta \longrightarrow+\infty} G(\eta)=1-\varepsilon, \quad \lim _{\eta-\infty} G(\eta)=\frac{1}{2}-4 \varepsilon .
$$

We now fix the constant $\theta$ by requiring that $G(0)=\frac{1}{2}-2 \varepsilon$, i.e. $\theta=\frac{1-\varepsilon}{2 \varepsilon}$. Routine calculations give $G\left(\eta_{0}\right)=1 / 2-3 \varepsilon$, with

$$
\eta_{0}=\frac{2}{1+6 \varepsilon} \log \frac{1-\varepsilon}{1+4 \varepsilon} .
$$

We then define

$$
h(\eta, \sigma)=G(\eta-\gamma-c(\sigma-\bar{s}))
$$


with $\gamma=-\eta_{0}+\tau^{2}$. By construction, $h$ satisfies (5.52), $h_{\eta} \geq 0$ and $h(\eta, \sigma) \leq 1-\varepsilon$. Moreover, at $\sigma=\bar{s}$

$$
h(\eta, \bar{s})=G(\eta-\gamma) \leq \frac{1}{2}-3 \varepsilon \leq \frac{1}{2}-2 \varepsilon<\psi(\eta, \bar{s}) \text { for all } \eta \in\left(0, \tau^{2}\right)
$$

(by our choice of $\eta_{0}$ ). On the other hand, if $\sigma \geq \bar{s}$ is such that

$$
-c(\sigma-\bar{s}) \leq \gamma,
$$

then at $\eta=0$ we have

$$
h(0, \sigma)=G\left(-\gamma-c(\sigma-\overline{\bar{s}}) \leq \frac{1}{2}-2 \varepsilon .\right.
$$

Therefore, we deduce by comparison that $\psi(\eta, \sigma) \geq h(\eta, \sigma)-\varepsilon / 4$ for all $\eta$ in $\left(0, \tau^{2}\right)$ and all $\sigma \in[\bar{s}, s]$ satisfying (5.58). Since $\bar{\tau}<\tau$, we have $-c(s-\bar{s}) \leq \gamma$, and then

$$
\psi(\eta, s) \geq \underline{\psi}(\eta, s) \geq h(\eta, s)-\frac{\varepsilon}{4} \quad \text { for } \eta \in\left(0, \tau^{2}\right) .
$$

Finally, observe that if, say, $\eta \geq-2 \log \varepsilon$, then

$$
G(\eta) \geq 1-\frac{3 \varepsilon}{2} \text {. }
$$

Therefore, we have

$$
\psi(\eta, s) \geq h(\eta, s)-\frac{\varepsilon}{4}=G\left(\eta+\eta_{0}-\tau^{2}+\bar{\tau}^{2}\right)-\frac{\varepsilon}{4} \geq 1-7 \varepsilon / 4,
$$

provided that

$$
\eta \geq-2 \log \varepsilon-\eta_{0}+\tau^{2}-\bar{\tau}^{2} .
$$

Observe finally that if $\tau_{0}$ is sufficiently large, $\tau^{2}-\bar{\tau}^{2} \leq 1$. This follows from $(\tau-\bar{\tau}) \leq e^{-\frac{\bar{\tau}}{2}}$, or, in terms of the variable $s$,

$$
\frac{s}{\bar{s}}=1+\frac{\log ^{2} \bar{s}}{\bar{s}} \leq e^{\frac{1}{\sqrt{\bar{s}}}}=1+\frac{1}{\sqrt{\bar{s}}}+\cdots,
$$

which certainly holds for $\bar{s}$ sufficiently large. Using (5.48c), the lemma follows with $R(\varepsilon)$ given by

$$
R(\varepsilon)=-2 \log \varepsilon-\frac{2}{1+6 \varepsilon} \log \frac{1-\varepsilon}{1+4 \varepsilon}+1 .
$$

We next improve the estimate for $\Psi$ on the interval $(-R(\varepsilon), R(\varepsilon))$. In order to state our next result, consider the travelling wave solutions of the equation

$$
Z_{s}=Z_{\eta \eta}+(2 Z-\gamma) Z_{\eta}
$$

where $\gamma$ is a free parameter. These are of the form $Z(\eta, s)=H(\eta-c(s-\bar{s}))$ for some constant $c$, where $H$ satisfies the equation

$$
\begin{gathered}
H(\eta) \equiv H_{1-\beta_{2}, \eta_{0}, \beta_{1}}=\frac{\beta_{2}+\beta_{1} e^{\left(\beta_{1}-\beta_{2}\right)\left(\eta-\eta_{0}\right)}}{1+e^{\left(\beta_{1}-\beta_{2}\right)\left(\eta-\eta_{0}\right)}}, \\
\beta_{1}+\beta_{2}=\gamma-c, \quad \beta_{1} \beta_{2}=c
\end{gathered}
$$

and $\eta_{0}$ is an arbitrary integration constant. Observe that if $\beta_{1} \geq \beta_{2}$ then

$$
\lim _{\eta \longrightarrow \infty} H(\eta)=\beta_{1}, \quad \lim _{\eta \rightarrow \infty} H(\eta)=\beta_{2} .
$$


Notice also that for every constant $c \in(0, \gamma / 2)$ we can choose $\beta_{1}=\gamma-c, \beta_{2}=0$ and $\eta_{0}=(\log (\gamma-2 c)) /(\gamma-c)$ so that $H(0)=1 / 2$. When $\gamma=1$ this solution will be denoted by $H_{c}$. We can now state the following lemma.

Lemma 5.8. Let $\Psi$ be the function given in (5.37) and $R=R(\varepsilon)$ be the constant given by Lemma 5.6. Then there is a $\sigma=\sigma(\varepsilon)$ such that, for every $s \in\left[\sigma_{0}, \sigma_{1}\right]$ satisfying

$$
s \geq \sigma_{0}+\frac{2}{\pi \varepsilon^{2}} \log ^{4} \sigma_{0}+\log ^{2} \sigma_{0}+\sigma(\varepsilon),
$$

we have

$$
\left|\Psi(z, \sigma)-H_{0}(z)\right| \leq 12 \varepsilon \text { for all } z \in(-R, R),
$$

where $H_{0}$ is the unique solution of (5.61), with $c=0, \gamma=1$ such that $H_{0}(0)=1 / 2$.

Proof. The proof is based again on a comparison argument in a time interval $[\bar{s}, s]$, where $s$ is any fixed time of $\left(\sigma_{0}, \sigma_{1}\right)$ satisfying (5.63). The conditions on $\bar{s}$ will be imposed as we proceed with the proof. We begin by taking $\bar{s}$ such that

$$
\bar{s} \in\left(\sigma_{0}+\frac{2}{\pi \varepsilon^{2}} \log ^{4} \sigma_{0}+\log ^{2} \sigma_{0}, s\right)
$$

and such that conditions (5.46) and (5.49) are fulfilled. We then consider the following auxiliary function $\bar{\psi}$ on the region $\eta \in(0, R), \sigma \in[\bar{s}, s]$

$$
\bar{\psi}(\eta, \sigma)=K_{M} \log \left(\frac{\sigma}{\bar{s}}\right)+g(\eta, \sigma)
$$

where $K_{M}$ is a constant such that $K_{M}=O\left(C_{M}\right)$. As before, if $\tau_{0}$ is picked sufficiently large we have

$$
\left|K_{M} \log \frac{\sigma}{\bar{s}}\right| \leq \frac{\varepsilon}{4}
$$

and therefore, in order for $\bar{\psi}$ to be a supersolution, we only need g to satisfy

$$
g_{\sigma}=g_{\eta \eta}+(2 g-(1-\varepsilon)) g_{\eta}, \quad \text { on } \quad(0, R),
$$

$$
g_{\eta} \geq 0, \quad \text { on } \quad(0, R) .
$$

Moreover, we also need the following boundary and initial conditions to hold

$$
\begin{gathered}
\psi(\eta, \bar{s}) \leq g(\eta, \bar{s}) \quad \text { for } \eta \in(0, R), \\
\psi(0, \sigma) \leq g(0, \sigma), \quad \psi(R, s)<g(R, s) .
\end{gathered}
$$

By the choice of $s$ and $\bar{s},(5.48)$, and Lemma 5.4, we have

$$
\psi(R, \sigma) \leq 1+\varepsilon, \quad \psi(0, \sigma) \leq \frac{1}{2}+\varepsilon
$$

for every $\sigma \in[\bar{s}, s]$. Therefore, we are interested in solutions of (5.65) such that

$$
g(R, \sigma)=1+\varepsilon, \quad g(0, \sigma)=\frac{1}{2}+\varepsilon .
$$

Given any initial data $g(\eta, \bar{s})$, problem $(5.65 \mathrm{a}),(5.67)$ obviously has a unique classical global solution on $(0, R)$. Moreover, if $g(\eta, \bar{\sigma})$ is monotone increasing on $(0, R)$, then the solution $g(\cdot, \sigma)$ is monotone increasing on $(0, R)$ for all times by the maximum principle. By the estimates obtained on $\psi$ and $\psi_{\eta}$ when $\sigma$ is in $\left(\sigma_{0}, \sigma_{1}\right)$ it is possible to construct a monotone increasing function $\bar{g}$ satisfying $\bar{g}(0)=\varepsilon+1 / 2$ 
and $\bar{g}(R)=1+\varepsilon$, and such that for all $\sigma$ in $\left(\sigma_{0}, \sigma_{1}\right)$ one has $\psi(\eta, \sigma) \leq \bar{g}$ on $(0,+\infty)$. We take that function as initial value, i.e. we set

$$
g(\eta, \bar{s})=\bar{g}(\eta) .
$$

We are interested in the behaviour of that solution as $\sigma$ increases. This can be easily described, since we have a Lyapunov functional which is strictly decaying along the trajectories of the solutions. Indeed, let $\mathrm{H}^{+}$be the stationary solution of (5.61) with $\gamma=1-\varepsilon / 2$ and the boundary conditions (5.62)

$$
H^{+}(\eta)=\frac{\beta_{2}+\beta_{1} e^{\left(\beta_{1}-\beta_{2}\right)\left(\eta+\eta_{0}\right)}}{1+e^{\left(\beta_{1}-\beta_{2}\right)\left(\eta-\eta_{0}\right)}}
$$

where $\beta_{1}, \beta_{2}$ and $\eta_{0}$ are such that

$$
\beta_{1}+\beta_{2}=1-\frac{\varepsilon}{2}, \quad H^{+}(0)=\frac{1}{2}+\varepsilon, \quad \text { and } \quad H^{+}(R)=1+\varepsilon .
$$

Since $H^{+}$is monotone increasing, we necesarily have $\beta_{1}>1+\varepsilon$, and thus $\beta_{2}<$ $-3 \varepsilon / 2$. It is easy to check that the values of $\beta_{1}$ and $\eta_{0}$ are related as follows

$$
\eta_{0}=\frac{1}{2 \beta_{1}-1+\varepsilon / 2} \log \left(\frac{2 \beta_{1}-1+3 \varepsilon}{2 \beta_{1}-1-2 \varepsilon}\right)=\frac{1}{2 \beta_{1}-1+\varepsilon / 2} \log \left(\frac{2 \beta_{1}+3 \varepsilon}{\beta_{1}-1-\varepsilon}\right)>0 .
$$

Consider then the functional

$$
J(\sigma)=\int_{0}^{R}\left(g(\eta, \sigma)-H^{+}(\eta)\right)^{+} d \eta .
$$

It follows from Kato's inequality that $J^{\prime}(s)<0$. Standard results on the asymptotic behaviour of parabolic equations on bounded domains then give

$$
\lim _{s \longrightarrow \infty}\left\|g(\cdot, s)-H^{+}(\cdot)\right\|_{\infty}=0 .
$$

In particular, there exists a $\sigma(\varepsilon)$ such that if $\sigma>\bar{s}+\sigma(\varepsilon)$ then

$$
\left|g(\eta, s)-H^{+}(\eta)\right| \leq \frac{\varepsilon}{4} \quad \text { for } 0<\eta<R
$$

We next observe that, by (5.63), it is possible to have $\sigma \in[\bar{s}, s]$ satisfying $\sigma \geq$ $\bar{s}+\sigma(\varepsilon)$. Actually, it is enough to have $s-\bar{s}>\sigma(\varepsilon)$ or, equivalently, $\tau-\bar{\tau} \geq 2 \sigma(\varepsilon) e^{-\bar{\tau}}$. Since $\sigma(\varepsilon)$ only depends on $\varepsilon, R$, and the initial data $\bar{g}$, but not on $s$ nor $\bar{s}$, this holds if $\tau_{0}$ is sufficiently large. Similarly, one can construct a subsolution

$$
\underline{\psi}(\eta, \sigma)=-K_{M} \log \left(\frac{\sigma}{\bar{s}}\right)+h(\eta, \sigma)
$$

where $h$ satisfies the following initial boundary value problem

$$
\begin{gathered}
h_{\sigma}=h_{\eta \eta}+(2 h-(1+\varepsilon)) h_{\eta}, \quad \text { on } \quad(0, R), \\
h_{\eta} \geq 0, \quad \text { on } \quad(0, R), \\
h(\eta, \bar{s})=\underline{h} \leq \psi(\eta, \bar{s}), \\
h(0, \sigma)=\frac{1}{2}-\varepsilon, \quad h(R, \sigma)=1-\varepsilon .
\end{gathered}
$$

The function $\underline{h}$ is constructed in a similar way to $\bar{g}$. It is monotone increasing in $\left(\sigma_{0}, \sigma_{1}\right), \underline{h}(0)=-\varepsilon+1 / 2, \underline{h}(R)=1-\varepsilon$, and is such that for all $\sigma \in\left(\sigma_{0}, \sigma_{1}\right)$, $\psi(\eta, \sigma) \geq \underline{h}$ on the interval $(0, R)$. By the same argument as before there is a 
positive time, which without any loss of generality we can choose equal to $\sigma(\varepsilon)$, such that if $\sigma>\sigma(\varepsilon)$, then

$$
\left|h(\eta, \sigma)-H^{-}(\eta)\right| \leq \frac{\varepsilon}{4} \quad \text { for } 0<\eta<R,
$$

where $H^{-}$is the stationary solution of (5.61) with $\gamma=1+\varepsilon / 2$ and the boundary conditions (5.70). In order to estimate the diference $\left|\psi(\eta, \sigma)-H_{0}(\eta)\right|$ we point out that

$$
\psi(\eta, \sigma)-H_{0}(\eta) \leq K_{M} \log \frac{\sigma}{\bar{s}}+g(\eta, \sigma)-H^{+}(\eta)+H^{+}(\eta)-H_{0}
$$

and

$$
\psi(\eta, \sigma)-H_{0}(\eta) \geq-K_{M} \log \frac{\sigma}{\bar{s}}+h(\eta, \sigma)-H^{-}(\eta)+H^{-}(\eta)-H_{0} .
$$

By (5.56), if $\sigma-\bar{s} \geq \sigma(\varepsilon)$, then

$$
\left|g(\eta, \sigma)-H^{+}(\eta)\right| \leq \frac{\varepsilon}{4}
$$

and therefore

$$
\left|K_{M} \log \frac{\sigma}{\bar{s}}+g(\eta, \sigma)-H^{+}(\eta)\right| \leq \frac{\varepsilon}{2} .
$$

On the other hand, under the same conditions

$$
\left|-K_{M} \log \frac{\sigma}{\bar{s}}+h(\eta, \sigma)-H^{-}(\eta)\right| \leq \frac{\varepsilon}{2} .
$$

Hence

$$
\left|\psi(\eta, \sigma)-H_{0}(\eta)\right| \leq \frac{\varepsilon}{2}+\max \left(\left|H_{0}(\eta)-H^{+}(\eta)\right|,\left|H_{0}(\eta)-H^{-}(\eta)\right|\right)
$$

Now let us estimate the difference $\left|H^{ \pm}(\eta)-H_{0}(\eta)\right|$. By definition, $H_{0}(\eta)=\frac{1}{1+e^{-\eta}}$. Recalling our choice of $H^{+}$in (5.68a), it is easy to check that the values of $\beta_{1}$ and $\eta_{0}$ are such that $\beta_{1}>1+\varepsilon, \beta_{2}<-3 \varepsilon / 2$ and

$$
1+\varepsilon=\frac{\left(1-\beta_{1}-\varepsilon / 2\right)+\beta_{1} e^{\left(\beta_{1}-\beta_{2}\right)\left(R+\eta_{0}\right)}}{1+e^{\left(\beta_{1}-\beta_{2}\right)\left(R+\eta_{0}\right)}} \geq \frac{\left(1-\beta_{1}-\varepsilon / 2\right)+\beta_{1} e^{\left(\beta_{1}-\beta_{2}\right) R}}{1+e^{\left(\beta_{1}-\beta_{2}\right) R}},
$$

whence

$$
\frac{e^{R}(1+\varepsilon)+3 \varepsilon / 2}{e^{R}-1} \geq \beta_{1}>1+\varepsilon,
$$

and

$$
\varepsilon<\beta_{1}-1 \leq \varepsilon+\frac{1+5 \varepsilon / 2}{e^{R}-1}
$$

Using (5.59), it now turns out that

$$
\varepsilon<\beta_{1}-1 \leq \frac{5 \varepsilon}{2}, \quad-3 \varepsilon \leq \beta_{2} \leq \frac{3 \varepsilon}{2} .
$$


We then have

$$
\begin{aligned}
H^{+}(\eta)-H_{0}(\eta) & =\frac{\beta_{1}+\beta_{2} e^{-\left(\beta_{1}-\beta_{2}\right)\left(\eta+\eta_{0}\right)}}{1+e^{-\left(\beta_{1}-\beta_{2}\right)\left(\eta+\eta_{0}\right)}}-\frac{1}{1+e^{-\eta}} \\
& =\left(\frac{\beta_{1}+\beta_{2} e^{-\left(\beta_{1}-\beta_{2}\right)\left(\eta+\eta_{0}\right)}}{1+e^{-\left(\beta_{1}-\beta_{2}\right)\left(\eta+\eta_{0}\right)}}-\frac{1}{1+e^{-\left(\beta_{1}-\beta_{2}\right)\left(\eta+\eta_{0}\right)}}\right) \\
& +\left(\frac{1}{1+e^{-\left(\beta_{1}-\beta_{2}\right)\left(\eta+\eta_{0}\right)}}-\frac{1}{1+e^{-\eta}}\right) \\
& \left.\leq\left(\beta_{1}-1\right)-\beta_{2}\right)+\left(e^{-\eta}-e^{-\left(\beta_{1}-\beta_{2}\right)\left(\eta+\eta_{0}\right)}\right) .
\end{aligned}
$$

By the mean value theorem,

$$
\left|e^{-\eta}-e^{-\left(\beta_{1}-\beta_{2}\right)\left(\eta+\eta_{0}\right)}\right|=\left|\left(\eta-\left(\beta_{1}-\beta_{2}\right)\left(\eta+\eta_{0}\right)\right)\right| e^{-\left(\rho x+(1-\rho)\left(\beta_{1}-\beta_{2}\right)\left(\eta+\eta_{0}\right)\right)},
$$

for some $\rho \in(0,1)$, and, since $\beta_{1}-\beta_{2}>0$ and $\eta_{0}>0$,

$$
\begin{aligned}
\left|e^{-\eta}-e^{-\left(\beta_{1}-\beta_{2}\right)\left(\eta+\eta_{0}\right)}\right| & \leq\left(\eta\left(\beta_{1}-1\right)-\beta_{2}\left(\eta+\eta_{0}\right)\right) e^{-\left(\rho \eta+(1-\rho)\left(\eta+\eta_{0}\right)\right)} \\
& \leq\left(\sup _{x>0} x e^{-x}\right)\left(\beta_{1}-1-\beta_{2}\right) \leq \frac{11}{2 e} \varepsilon
\end{aligned}
$$

Arguing in the same way with $H^{-}$, we obtain, for every $\eta \in(0, R)$,

$$
\left|H^{ \pm}(\eta)-H_{0}(\eta)\right| \leq \frac{11}{e} \varepsilon
$$

and, taking advantage of (5.48), the lemma follows.

Lemma 5.8 allows us to linearize equation $(5.43 \mathrm{~b})$ around a particular travelling wave solution of the equation (5.60), but we need to be careful when choosing the speed $c$ of the travelling wave in order to be able to fulfill the appropriate boundary conditions. To this end we shall make use of some auxiliary functions. Let $W(\eta)$ be the solution of

$$
\left\{\begin{array}{c}
W^{\prime \prime}+\left(2 H_{0}-1\right) W^{\prime}+2 H_{0}^{\prime}=-\left(\left(2 H_{0}-1\right) H_{0}-\eta H_{0}^{\prime}+H_{0}^{\prime}\right) \\
\equiv \frac{1-\eta e^{-\eta}}{\left(1+\eta e^{-\eta}\right)^{2}} \text { on } \mathbf{R} \\
W(0)=0, \quad W(\eta)=o(1) \quad \text { as } \eta \longrightarrow-\infty \\
W(\eta)=\eta+C_{\infty} \text { as } \eta \longrightarrow+\infty
\end{array}\right.
$$

By using the variation of constants formula, it turns out that problem (5.72) has a unique solution for a suitable value of the constant $C_{\infty}$. Let $Q(\eta)$ be defined as the solution of the problem

$$
\left\{\begin{array}{l}
Q^{\prime \prime}+\left(2 H_{0}-1\right) Q^{\prime}+2 H_{0}^{\prime} Q=-H_{0}^{\prime} \quad \text { on } \quad \mathbf{R} \\
Q(0)=0, \quad Q(-\infty)=0
\end{array}\right.
$$

The existence and uniqueness of such a solution is clear by linear ODE theory. Indeed, the general solution of the equation can be written in the form

$$
Q=Q_{p}+C_{1} \phi_{1,1}+C_{2} \phi_{1,2}
$$

where $Q_{p}$ is a particular solution and $\phi_{1,1}, \phi_{1,2}$ are two independent solutions of the homogeneous equation defined in (4.44), namely,

$$
\phi_{1,1}=\frac{1+2 \eta e^{-\eta}-e^{-2 \eta}}{\left(1+e^{-\eta}\right)^{2}}, \quad \phi_{1,2}=\frac{e^{-\eta}}{\left(1+e^{-\eta}\right)^{2}} .
$$


Therefore every solution $Q$ is determined by its values at 0 and $-\infty$. This in turn fixes the value

$$
\Gamma_{1}=Q(+\infty)
$$

Actually,

$$
Q(\eta)=\frac{e^{-\eta}-1-\eta e^{-\eta}}{\left(1+e^{-\eta}\right)^{2}}
$$

and $\Gamma_{1}=-1$. We now prove

Lemma 5.9. Let $H_{\alpha+c, \eta_{0}, \alpha}$ be the solution of

$$
\left\{\begin{array}{l}
-c H^{\prime}=H^{\prime \prime}+(2 H-1) H^{\prime} \quad \text { on } \quad \mathbf{R}, \\
H(-\infty)=\alpha, \quad H(+\infty)=1-c-\alpha,
\end{array}\right.
$$

as given in (5.62), and define

$$
\triangle=|\alpha|^{2}+|c|^{2}+\left|\eta_{0}\right|^{2} .
$$

Then

$$
H_{c}(\eta)=H_{0}-c Q-\eta_{0} \phi_{1,2}-\alpha \phi_{1,1}+O\left(\frac{e^{-\eta}}{1+e^{-\eta}}\left|\triangle^{1 / 2}\right|\left(1+|\eta|^{2}\right)\right),
$$

in the region

$$
(|\alpha|+|c|)|\eta|^{4} \leq 1 \quad \text { and }\left|\eta_{0}\right| \leq 1, \quad \triangle \sim e^{-\tau} \quad \text { and }|\eta| \sim \tau^{2} .
$$

Proof. The function $H_{c}$ is given by

$$
H_{c}(\eta)=\frac{(1-c-\alpha)+\alpha e^{(2 \alpha+c-1)\left(\eta-\eta_{0}\right)}}{1+e^{(2 \alpha+c-1)\left(\eta-\eta_{0}\right)}} .
$$

Using Taylor's expansion, we get

$$
\begin{aligned}
& H_{c}(\eta)=H_{0}-c Q-\eta_{0} \phi_{1,2}-\alpha \phi_{1,1}-2 c \frac{1+\eta e^{-\eta}}{\left(1+e^{-\eta}\right)^{2}}+O\left(\frac{e^{-\eta}}{1+e^{-\eta}} \triangle\left(1+|\eta|^{2}\right)\right) \\
& =H_{0}-c Q-\eta_{0} \phi_{1,2}-\alpha \phi_{1,1}+O\left(\frac{e^{-\eta}}{1+e^{-\eta}} \triangle^{1 / 2}\left(1+|\eta|^{2}\right)\right) .
\end{aligned}
$$

The lemma follows.

As a next step, we establish

Lemma 5.10. There exist a positive constant $C=C(M, \varepsilon)$, independent of $s$, such that, for $s \in\left(\sigma_{0}, \sigma_{1}\right)$ satisfying

$$
s \geq 2 \sigma_{0}+\frac{2}{\pi \varepsilon^{2}} \log ^{4} \sigma_{0}+\log ^{2} \sigma_{0}+\sigma(\varepsilon) \sigma,
$$

we have

$$
\left|\Psi(z, s)-H_{0}(z)-\frac{W(z)}{s}-c(s) Q(z)-K(s) \phi_{1,1}(z)\right| \leq C \frac{\log s}{s}, \quad \text { if } \quad|z| \leq \log ^{2} s,
$$

where $c(s)$ and $K(s)$ are given in (5.79) below. 
Proof. Let us take $s \in\left(\sigma_{0}, \sigma_{1}\right)$ and select $\bar{s} \geq \sigma_{0}+\frac{2}{\pi \varepsilon^{2}} \log ^{4} \sigma_{0}+\log ^{2} \sigma_{0}+\sigma(\varepsilon)$ such that

$$
\mu(\log s-\log \bar{s})=\frac{\log \bar{s}}{\bar{s}} .
$$

Clearly, if $\sigma_{0}$ is sufficiently large, $s$ and $\bar{s}$ satisfy the conditions (5.46) and (5.49). On the other hand, simple calculus arguments show first that

$$
\lim _{0} \longrightarrow+\infty \frac{s-\bar{s}}{\bar{s}} e^{\mu(s-\bar{s})}=1,
$$

and moreover that if $\sigma_{0}$ is large enough there is a positive constant $C$ such that

$$
\frac{s-\bar{s}}{\bar{s}} \leq C \frac{\log s}{s} .
$$

Observe that the first of these two properties implies that, for $\sigma_{0}$ suficiently large,

$$
s-\bar{s} \geq \frac{\sigma_{0}}{2} e^{-\mu(s-\bar{s})} .
$$

Then we will have $s-\bar{s} \geq m$ for any positive constant $m$ such that $2 m \leq \sigma_{0} e^{-\mu m}$. In particular, we can assume $s-\bar{s}$ to be arbitrarily large if we take $\sigma_{0}$ sufficiently large. We then define

$$
Z(\eta, \sigma)=\psi(\eta, \sigma)-H_{0}(\eta)-\frac{W(\eta)}{\sigma}-c(s) Q(\eta)-K(s) \phi_{1,1}(\eta)
$$

and proceed to select the constants $c(\bar{s})$ and $K(\bar{s})$. This can be done by looking at the values of the right hand side in (5.76) at the points $\eta= \pm \log ^{2} s$. Since $s$ and $\bar{s}$ satisfy (5.46) and (5.49) if $\sigma_{0}$ is large enough, we have by (5.47)

$$
\begin{aligned}
& \left|\psi\left(\log ^{2} s, \sigma\right)-\left(1-\lambda(\log s)+\frac{C_{1}}{\sigma}\right)+\frac{\log ^{2} s}{\sigma}\right| \leq \frac{3 \varepsilon}{s}, \\
& \left|\psi\left(\log ^{2} s, \sigma\right)-\frac{C_{2}}{\bar{s}}\right| \leq \frac{3 \varepsilon}{s},
\end{aligned}
$$

for $\sigma \in[\bar{s}, s]$. On the other hand, as we have seen,

$$
\begin{aligned}
& \left|W\left(\log ^{2}(s)\right)-\log ^{2}(s)-C_{\infty}\right|+\left|H_{0}\left(\log ^{2} s\right)-1\right| s \\
& +\left|Q\left(\log ^{2}(s)\right)+1\right| s+\left|\phi_{1,1}\left(\log ^{2}(s)\right)-1\right| s=o(1) \quad \text { as } s \longrightarrow+\infty, \\
& \left|W\left(-\log ^{2}(s)\right)\right|+\left|H_{0}\left(-\log ^{2} s\right)\right| s+\left|Q\left(-\log ^{2}(s)\right)\right| s \\
& +\left|\phi_{1,1}\left(-\log ^{2}(s)\right)+1\right| s=o(1) \quad \text { as } s \longrightarrow+\infty .
\end{aligned}
$$

We now take $c(s)$ and $K(s)$ such that

$$
K+\frac{C_{2}}{s}=0, \quad c=\lambda(\log s)-\frac{C_{1}}{s}+\frac{C_{\infty}}{s}-K .
$$

With this choice of $c(s)$ and $K(s)$ we have, for every $\sigma \in[\bar{s}, s]$,

$$
\left|Z\left( \pm \log ^{2} s, \sigma\right)\right| \leq \frac{4 \varepsilon}{s},
$$

whereas by $(5.49)$

$$
|Z(0, \sigma)| \leq \frac{C^{\prime}(M)(\sigma-\bar{s})}{\bar{s}}+\frac{1}{4 \bar{s}} .
$$

On the other hand, we claim that if $\sigma_{0}$ is sufficiently large, then

$$
|Z(\eta, \sigma)| \leq 15 \varepsilon \quad \text { on }\left(-\log ^{2} s, \log ^{2} s\right), \text { for } \sigma \in[\bar{s}, s] .
$$


In order to prove (5.81), we remark that for every $\eta \in(-R, R)$

$$
\begin{aligned}
|Z(\eta, \sigma)| & \leq\left|\psi(\eta, \sigma)-H_{0}(\eta)\right|+\left|\frac{W(\eta)}{\sigma}-c(s) Q(\eta)-K(s) \phi_{1,1}(\eta)\right| \\
& \leq|\psi(\eta, \sigma)-\Psi(z, \sigma)|+\left|\Psi(z, \sigma)-H_{0}(z)\right|+\left|H_{0}(z)-H_{0}(\eta)\right| \\
& +\left|\frac{W(\eta)}{\sigma}-c(s) Q(\eta)-K(s) \phi_{1,1}(\eta)\right| .
\end{aligned}
$$

We then conclude by using (5.48c), (5.76) and the behaviour of $W, Q$ and $\Phi_{11}$ at $\pm \infty$.

For $|\eta| \geq R$ we write

$$
|Z(\eta, \sigma)| \leq\left|\psi(\eta, \sigma)-H_{c}(\eta)\right|+\left|H_{c}(\eta)-H_{0}-c Q\right|+\left|\frac{W(\eta)}{s}\right|+|K|\left|\phi_{11}(\eta)\right| .
$$

By our choice of $\bar{s}$, Lemma 5.3 and Lemma 5.5 we have that, for every $\eta \in\left(R, \log ^{2} s\right)$ and $\eta \in\left(-\log ^{2} s,-R\right)$,

$$
\psi(\eta, \sigma) \in\left(1-\frac{3 \varepsilon}{2}, 1+\varepsilon\right)
$$

and

$$
H_{c}(\eta) \in\left(\frac{1-c}{(1-2 c) e^{-(1-c) R}+1}, 1-c\right) .
$$

We then can choose $\sigma_{0}$ and $R$ so large that $c(s)<\varepsilon / 8$ and

$$
\frac{1-c}{(1-2 c) e^{-(1-c) R}+1} \geq 1-\frac{\varepsilon}{8}
$$

From this we deduce that

$$
\left|\psi(\eta, s)-H_{c}(\eta)\right| \leq \frac{5}{4} \varepsilon .
$$

On the other hand, by Lemma 5.9

$$
\left|H_{c}(\eta)-H_{0}-c Q\right| \leq c K \leq \frac{\varepsilon}{4},
$$

for $\sigma_{0}$ large enough. Finally,

$$
\left|\frac{W(\eta)}{\sigma}\right|+|K|\left|\phi_{11}(\eta)\right| \leq 2 \frac{\log ^{2} s+C_{\infty}}{\bar{s}}+|K(s)|,
$$

and (5.81) follows. Using this estimate, $Z$ satisfies the following equation

$$
\begin{aligned}
e^{-\tau} Z_{\tau}= & Z_{\eta \eta}+\left(2 H_{0}-1\right) Z_{\eta}+2 H_{0 \eta} Z+2 Z Z_{\eta}+2 e^{-\tau} W Z_{\eta} \\
& +2 W_{\eta} Z e^{-\tau}+e^{-\tau}\left(4 H_{0} Z+2 Z^{2}-Z-\eta Z_{\eta}+Z_{\eta}\right)+O\left(e^{-3 \tau / 2}\right),
\end{aligned}
$$

with initial and boundary conditions

$$
\begin{gathered}
|Z(\eta, \bar{s})| \leq 15 \varepsilon \quad \text { for any } \eta \in\left(-\log ^{2} s,+\log ^{2} s\right), \\
\left|Z\left( \pm \log ^{2} s, \sigma\right)\right| \leq \frac{2 \varepsilon}{s} \quad \text { for } \sigma \in(\bar{s}, s) .
\end{gathered}
$$

Moreover, by (5.81) and classical parabolic estimates, we also obtain that, for some positive constant $C$,

$$
\left|Z_{\eta}\right|+\left|Z_{\eta \eta}\right|+\left|Z_{\tau}\right| \leq C \varepsilon \quad \text { for } \quad \eta \in\left(-\log ^{2} s, \log ^{2} s\right) \quad \text { and } \sigma \in(\bar{s}, s) .
$$


Then, since

$$
\begin{gathered}
2 W_{\eta} e^{-\tau}+4 e^{-\tau} H_{0}+2 Z e^{-\tau}=O\left(e^{-5 \bar{\tau} / 6}\right), \\
2 e^{-\tau} W-e^{-\tau} \eta+e^{-\tau}=O\left(e^{-5 \bar{\tau} / 6}\right),
\end{gathered}
$$

equation (5.82a) can be rewritten as

$$
\begin{gathered}
e^{-\bar{\tau}} Z_{\tau}=Z_{\eta \eta}+\left(2 H_{0}-1\right) Z_{\eta}+Z\left(2 H_{0 \eta}+O\left(e^{-5 \bar{\tau} / 6}\right)\right) \\
+Z_{\eta}\left(2 Z+O\left(e^{-5 \tau / 6}\right)\right)+O\left(e^{-3 \tau / 2}\right)
\end{gathered}
$$

In order to prove Lemma 5.10. we provide a supersolution of (5.83), (5.82b) (5.82c) using two kinds of auxiliary functions. These are as follows

(1) A function $Z_{1}(\eta)$, solving

$$
\left\{\begin{array}{l}
Z_{1}^{\prime \prime}+\left(2 H_{0}-1\right) Z_{1}^{\prime}+2 H_{0}^{\prime} Z_{1}=-\gamma, \quad \text { on } \quad(0,+\infty), \\
Z_{1}(0)=1, \quad Z_{1}^{\prime}(0)=0 .
\end{array}\right.
$$

where $\gamma$ is a sufficiently large positive constant. It can be easily checked that

$$
\left|Z_{1}\right| \leq C(1+\eta) \text { for } 0<\eta<+\infty .
$$

(2) A function $Z_{2}$ defined as follows

$$
\begin{aligned}
& Z_{2}^{\prime \prime}+\left(2 H_{0}-1+2 C \varepsilon\right) Z_{2}^{\prime}+2 H_{0}^{\prime} Z_{2}=-\mu Z_{2} \quad \text { for } 0<\eta<\log ^{2} s, \\
& Z_{2}(0)=\varepsilon, \quad Z_{2}\left(\log ^{2} s\right)=1 .
\end{aligned}
$$

where $\mu=(\log s)^{-4}$.

It is easily seen that, when $\varepsilon \longrightarrow 0$ and $\mu=0, Z_{2}$ approaches $\lambda(s) \Phi_{1,1}$, where $\lambda(s) \longrightarrow 1$ as $s \longrightarrow+\infty$. Notice that $Z_{2}^{\prime}>0$ on $\left(0, \log ^{2} s\right)$. Define

$$
\bar{Z}(\eta, \sigma)=\bar{s}^{-3 / 2} Z_{1}(\eta)+e^{-\mu(\sigma-\bar{s})} Z_{2}(\eta)
$$

Clearly, $\bar{Z}$ satisfies

$$
\left.e^{-\tau} \bar{Z}_{\tau} \geq \bar{Z}_{\eta \eta}+\left(2 H_{0}-1-Z\right)\right) \bar{Z}_{\eta}+2 H_{0, \eta} \bar{Z} .
$$

On the other hand, so long as $\frac{2}{\bar{s}} \leq e^{-\mu(\sigma-\bar{s})}$ holds, one has

$$
Z(\eta, \sigma) \leq \bar{Z}(\eta, \sigma) \text { for } 0<\eta<\log ^{2} s .
$$

Since $Z(\eta, \bar{s}) \leq \bar{Z}(\eta, \bar{s})$ for $\eta \in\left(0, \log ^{2} s\right)$, it follows from maximum principle that

$$
\bar{Z}(\eta, s) \geq Z(\eta, s) \text { for } 0<\eta \leq \log ^{2} s .
$$

Assume now in addition that $(s-\bar{s}) / \bar{s} \leq 2 e^{-\mu(s-\bar{s})}$. In view of (5.85) and (5.87), we now have

$$
Z(\eta, s) \leq C(M, \varepsilon) \frac{\log s}{s} \text { for } 0<\eta \leq \log ^{2} s,
$$

whereupon the result follows.

Next we prove 
Lemma 5.11. There is a positive constant $A(\varepsilon)$ such that, for every $s \in\left[\sigma_{0}, \sigma_{1}\right]$ satisfying

$$
s>2 \sigma_{0}+\frac{2}{\pi \varepsilon^{2}} \log ^{4} \sigma_{0}+\log ^{2} \sigma_{0}+\sigma(\varepsilon)+\log \sigma_{0}+\frac{8}{\sigma_{0}} \log \sigma_{0}
$$

and for all $|z| \leq \log ^{2} s$, one has

$$
\left|\Psi(z, s)-H_{0}(z)-\frac{W(z)}{s}-c(s) Q(z)-K(s) \phi_{1,1}(z)-W(0, s) \phi_{1,1}(z)\right| \leq \frac{A(\varepsilon)}{s},
$$

where $c(s)$ and $K(s)$ are given in (5.79).

Proof. We fix $s$ satisfying (5.88) and select $\bar{s}$ so that

$$
\bar{s}>2 \sigma_{0}+\frac{2}{\pi \varepsilon^{2}} \log ^{4} \sigma_{0}+\log ^{2} \sigma_{0}+\sigma(\varepsilon)
$$

and

$$
\log \bar{s}+\frac{8}{\bar{s}} \log \bar{s}=\log s
$$

If $\sigma_{0}$ is suficiently large, (5.46) and (5.49) hold. We then consider the function $Z(\eta, \sigma)$ obtained in the proof of the previous lemma. By (5.77) and (5.83), $Z$ satisfies

$$
Z_{\sigma}=Z_{\eta \eta}+\left(2 H_{0}-1\right) Z_{\eta}+2 H_{0}^{\prime} Z+O\left(\frac{1}{\bar{s}^{3 / 2}}\right)
$$

for $\sigma \in[\bar{s}, s]$ and $|z| \leq R^{\prime}(\varepsilon, s)$. Let us define

$$
Z=\bar{s}^{-3 / 2} \bar{Z}+\zeta
$$

where $\bar{Z}$ is a solution of

$$
\bar{Z}_{\sigma}=\bar{Z}_{\eta \eta}+\left(2 H_{0}-1\right) \bar{Z}_{\eta}+2 H_{0}^{\prime} \bar{Z}+O(1), \quad \bar{Z}(\eta, \bar{s})=0,
$$

and

$$
\left\{\begin{array}{l}
\zeta_{\sigma}=\zeta_{\eta \eta}+\left(2 H_{0}-1\right) \zeta_{\eta}+2 H_{0}^{\prime} \zeta+O(1) \\
\zeta(\eta, \sigma)=Z(\eta, \sigma), \quad \text { for }|\eta|=\log ^{2} s \\
\zeta(\eta, \bar{s})=Z(\eta, \bar{s}), \text { for }|\eta| \leq \log ^{2} s
\end{array}\right.
$$

The function $\bar{Z}$ can be bounded from above and from below by the sum of one function of the family defined in (5.84a) and another of the family defined in (5.84b), and can then be estimated as in the proof of the previous lemma. On the other hand, if we write

then $W$ satisfies

$$
\zeta(\eta, \sigma)=W(\eta, \sigma) \phi_{1,1}(\eta)
$$

$$
W_{\sigma}=W_{\eta \eta}+2 \frac{\phi_{1,1}^{\prime}}{\phi_{1,1}} W_{\eta}+\left(2 H_{0}-1\right) W_{\eta} \equiv W_{\eta \eta}-\operatorname{th}\left(\frac{\eta}{2}\right) W_{\eta},
$$

where we have used the fact that $\phi_{1,1}^{\prime \prime}+\left(2 H_{0}-1\right) \phi_{1,1}^{\prime}+2 H_{0}^{\prime} \phi_{1,1}=0$. Setting $q=W_{\eta}$, we easily check that $q$ is such that

$$
\left\{\begin{array}{l}
q_{\sigma}=q_{\eta \eta}-\left(\operatorname{th}\left(\frac{\eta}{2}\right) q\right)_{\eta}, \quad q(\eta, \bar{s})=\frac{\zeta_{\eta}}{\phi_{1,1}}-\frac{\phi_{1,1}^{\prime}}{\phi_{1,1}^{2}} \zeta \\
|q(\eta, \sigma)| \leq \frac{\varepsilon e^{|\eta|}}{\bar{s}}, \quad \text { if } \quad|\eta|=\log ^{2} s \\
\mid q\left(\eta, \bar{s} \mid \leq \frac{B_{M} \log \bar{s} s|\eta|}{\bar{s}} \quad \text { if } \quad|\eta| \leq \log ^{2} s\right.
\end{array}\right.
$$


The differential equation in (5.93) has a two parameter family of particular solutions. These are

$$
q_{1}(\eta, \sigma)=e^{-\frac{\sigma}{4}} \operatorname{ch}\left(\frac{\eta}{2}\right),
$$

as well as the stationary solutions

$$
\left\{\begin{array}{l}
q_{2}^{\prime \prime}-\left(\operatorname{th}\left(\frac{\eta}{2}\right) q_{2}\right)^{\prime}=0, \\
q_{2}(0)=1, \quad q_{2}^{\prime}(0)=0 .
\end{array}\right.
$$

It is easy to check that solutions of (5.93) satisfy $\eta q_{2}^{\prime}(\eta) \geq 0$ on $\mathbf{R}$ and $q_{2}(\eta) \sim K e^{|\eta|}$ as $|\eta| \longrightarrow+\infty$ for some given positive constant $K$. We now define

$$
\bar{q}(\eta, \sigma)=\frac{1}{\bar{s}}\left[A_{M} e^{-\frac{1}{4}(\sigma-\bar{s})} \operatorname{ch}\left(\frac{\eta}{2}\right) \log ^{k} \bar{s}+\frac{2 \varepsilon}{K} q_{2}(\eta)\right],
$$

where $k=k(r)=1+1 / 2 r$. By linearity, $\bar{q}$ is still a solution of the equation in (5.92). On the other hand

(1) For all $\eta \in\left(-\log ^{2} s, \log ^{2} s\right), \bar{q}(\eta, \bar{s}) \geq A_{M} \log ^{k} \frac{e^{\frac{|\eta|}{2}}}{2 \bar{s}}$. We then have $\bar{q}(\eta, \bar{s}) \geq$ $q(\eta, \bar{s})$, provided that $\log \bar{s} \geq C_{r}^{1 / 2 r} \frac{2 B_{M}}{A_{M}} \varepsilon^{-\frac{1}{2 r}}$.

(2) If $|\eta|=\log ^{2} s$, then $\bar{q}(\eta, \sigma) \geq \frac{2 \varepsilon}{K \bar{s}} q_{2}(\eta) \geq \frac{\varepsilon e^{R^{\prime}}}{\bar{s}} \geq q(\eta, \sigma)$.

By comparison we thus deduce that

$$
|q(\eta, s)| \leq \bar{q}(\eta, s)
$$

Integrating this inequality gives

$$
|W(\eta, \sigma)-W(0, \sigma)| \leq \frac{1}{\bar{s}}\left[A_{M}^{\prime} e^{-\frac{1}{4}(\sigma-\bar{s})} e^{\frac{|\eta|}{2}} \log ^{k} \bar{s}+C \varepsilon e^{|\eta|}\right],
$$

whence

$$
\left|\zeta(\eta, \sigma)-W(0, \sigma) \phi_{1,1}(\eta)\right| \leq \frac{1}{\bar{s}}\left[A_{M}^{\prime} e^{-\frac{1}{4}(\sigma-\bar{s})} e^{-\frac{|\eta|}{2}} \log ^{k} \bar{s}+\varepsilon C\right]
$$

for every $\sigma \in[\bar{s}, s]$. In particular, using our choice of $\bar{s}$,

$$
\begin{aligned}
& \left|\zeta(\eta, s)-W(0, s) \phi_{1,1}(\eta)\right| \leq \frac{1}{\bar{s}}\left[A_{M}^{\prime} e^{-\frac{1}{4}(\sigma-\bar{s})} e^{-\frac{|\eta|}{2}} \log ^{k} \bar{s}+\varepsilon C\right] \\
& \leq \frac{1}{\bar{s}}\left[\frac{\log ^{k+1} s}{s}+\varepsilon C\right] \leq \frac{1}{\bar{s}} \varepsilon C,
\end{aligned}
$$

and the lemma follows.

Remark. Observe that the function $W(0, \sigma)$ considered above is such that

$$
W(0, \bar{s})=\psi(0, \bar{s})=0 .
$$

The estimates that we have obtained so far are valid for any initial data $\phi \in$ $\mathcal{A}\left(M ; \tau_{0}, \tau_{1}\right)$, but they only hold true after some small time delay $\sigma \geq \rho\left(\sigma_{0}\right)$. In our next result we show that for suitable initial data, these estimates also hold true for $\sigma \geq \sigma_{0}$.

Lemma 5.12. Assume that $\phi \in \mathcal{A}\left(M ; \tau_{0}, \tau_{1}\right)$. Suppose that near the point $\xi=0$, the initial data $\phi\left(\xi, \tau_{0}\right)$ is taken to be one of the waves defined by (5.62). Then if $\tau_{0}$ is sufficiently large, (5.77) and (5.89) hold for all $\tau \in\left[\tau_{0}, \tau_{1}\right]$. 
Proof. As we have seen in the previous lemmas, there is a positive $\rho\left(\tau_{0}\right)$ such that (5.77) and (5.89) are satisfied provided that $\sigma_{0}$ is large enough and $\sigma \in\left[\rho\left(\tau_{0}\right), \sigma_{1}\right]$. The only thing which remains to be checked is that if we choose the initial data as in the statement of the lemma, then estimates (5.77) and (5.89) are also true for $\tau \in\left[\tau_{0}, \rho\left(\tau_{0}\right)\right]$. Observe that the estimates obtained in Lemmata 5.1-5.4 hold true for all $\tau \in\left[\tau_{0}, \tau_{1}\right]$. Moreover, let us choose $\phi\left(\tau_{0}\right)$ such that

$$
\Psi\left(z, \sigma_{0}\right)=H_{c\left(\tau_{0}\right), \eta_{0}, 0}(z)=\frac{\left(1-c\left(\tau_{0}\right)\right)}{1+e^{\left(c\left(\tau_{0}\right)-1\right)\left(z-\eta_{0}\right)}},
$$

in the region $|z| \leq 2 \tau_{0}^{2}$. We now select $\eta_{0}$ sufficiently small in the following way. By Lemma 5.9,

$$
\begin{aligned}
\left|\Psi\left(z, \sigma_{0}\right)-H_{0}(z)\right| & =\left|H_{c\left(\tau_{0}\right), \eta_{0}, 0}(z)-H_{0}(z)\right| \\
& \leq\left|c\left(\tau_{0}\right)\right||Q|+\left|\eta_{0}\right|\left|\phi_{1,1}(z)\right|+O\left(\frac{e^{-\eta}}{1+e^{-\eta}} \triangle^{1 / 2}\left(1+|\eta|^{2}\right)\right)
\end{aligned}
$$

in the region $\left|c\left(\tau_{0}\right)\right||\eta|^{4} \leq 1$ and $\left|\eta_{0}\right| \leq 1$, where $\triangle=\left|c\left(\tau_{0}\right)\right|^{2}+\left|\eta_{0}\right|^{2}$. Therefore, for every $\varepsilon>0$, there exists a positive constant $\delta$ such that if $\left|\eta_{0}\right|<\delta$ and $\tau_{0}$ is sufficiently large, then

$$
\left|\Psi\left(z, \sigma_{0}\right)-H_{0}(z)\right| \leq \varepsilon / 2
$$

for all $\eta$ in $\left(-2 \tau_{0}^{2}, 2 \tau_{0}^{2}\right)$. We are now in a position to apply the same arguments as in the proofs of Lemmata 5.10 and 5.11 to the function $\Psi(z, s)$ on the time interval $\left[\tau_{0}, \rho\left(\tau_{0}\right)\right]$ and deduce that first (5.77) and then (5.89) hold true on $\left[\sigma_{0}, \rho\left(\sigma_{0}\right)\right]$.

As a next step we obtain an estimate of the speed of the curves $\lambda(\tau)$.

Lemma 5.13. Assume that $\phi \in \mathcal{A}\left(M ; \tau_{0}, \tau_{1}\right)$ is a solution of (4.5) corresponding to admissible initial data such as those described before. Then there exist a constant $C_{1}$ and a function $g \equiv g(\tau)$ such that, for all $\tau \in\left[\tau_{0}, \tau_{1}\right]$,

$$
\left\{\begin{array}{l}
\frac{d \lambda}{d \tau}=\lambda(\tau)+C_{1} e^{-\tau}+\varepsilon g(\tau), \\
g(\tau)=O\left(e^{-\tau}\right) .
\end{array}\right.
$$

Proof. Fix any $\bar{s}<s$ such that $s-\bar{s} \leq \varepsilon$. We consider again the function $\psi$ defined by

$$
\phi(\xi, \tau)=\psi(\eta, \sigma), \quad \eta=(\xi-\lambda(\bar{\tau})) e^{\tau}, \quad \sigma=e^{\tau} .
$$

By Lemmata 5.10 and 5.11,

$$
\left|\psi(\eta, \bar{s})-H_{c(\bar{s}), \eta_{0}(\bar{s}), 0}(\eta)+e^{-\bar{s}} W(\eta)+W(0, \bar{s}) \phi_{1,1}(\eta)\right| \leq \frac{\varepsilon}{\bar{s}} .
$$

Moreover, $\psi$ satisfies the equation (5.43b), which we can write in the form

$$
\psi_{\sigma}=\psi_{\eta \eta}+(2 \psi-1) \psi_{\eta}+e^{-\tau}\left((2 \psi-1) \psi-\eta \psi_{\eta}+\psi_{\eta}\right)+O\left(e^{-2 \tau}\right)
$$

uniformly on $|\eta|<10^{6}$. We now claim that

$$
\psi(\eta, s)=H_{c(\bar{s}), \eta_{0}(\bar{s}), 0}(\eta-c(\bar{s})(s-\bar{s}))+\frac{W(\eta)}{\sigma}+O\left(\varepsilon e^{-\tau}\right) .
$$

In order to prove this claim we define the auxiliary function

$$
R(\eta, \sigma)=\psi(\eta, \sigma)-H_{c(\bar{s}), \eta_{0}(\bar{s}), 0}(\eta-c(\bar{s})(s-\bar{s}))+\frac{W(\eta)}{\sigma} .
$$


By the definition of $R$ and the remark above,

$$
R(\eta, \bar{s})=O\left(\varepsilon \frac{1}{\bar{s}}\right) .
$$

Moreover by Lemma 5.8 and the fact that $\tau-\bar{\tau} \leq \bar{\tau} e^{-\bar{\tau}}$ we have, uniformly on, say, $|\eta| \leq 1 / 2$,

$$
\begin{aligned}
\mid H_{0}(\eta) & -H_{c(\bar{s}), \eta_{0}(\bar{s}), 0}(\eta-c(\bar{s})(s-\bar{s})) \mid \\
\leq & \left|H_{0}(\eta)-H_{0}(\eta-c(\bar{s})(s-\bar{s}))\right| \\
& +\left|H_{0}(\eta-c(\bar{s})(s-\bar{s}))-H_{c(\bar{s}), \eta_{0}(\bar{s}), 0}(\eta-c(\bar{s})(s-\bar{s}))\right| \\
\leq & c(\bar{s})(s-\bar{s})\left|H_{0}^{\prime}\right|+c(\bar{s})|Q|+\eta_{0}(\bar{s})\left|\phi_{1,2}\right|+O\left(e^{-2 \tau}\right) \\
\leq & C_{M} e^{-\bar{\tau}}+C_{M}^{\prime}|\tau-\bar{\tau}| \leq C_{M} e^{-\bar{\tau}}
\end{aligned}
$$

Therefore

$$
|R(\eta, \sigma)| \leq C_{M} e^{-\bar{\tau}}
$$

Writing (5.43b) in terms of $R$ we now obtain

$$
\begin{aligned}
R_{\sigma}=R_{\eta \eta} & +(2 H-1) R_{\eta}+2 H^{\prime} R+e^{-\tau} W^{\prime \prime}(\eta) \\
& +(2 H-1) e^{-\tau} W^{\prime}(\eta)+2 H^{\prime} e^{-\tau} W \\
& +e^{-\tau}\left[(2 H-1) H-\eta H^{\prime}+H^{\prime}\right]+O\left(C_{M} e^{-2 \bar{\tau}}\right),
\end{aligned}
$$

where $H$ stands for $H_{c(\bar{s}), \eta_{0}(\bar{s}), 0}$. Observe that if we replace $H$ by $H_{0}$ in (5.98) we only introduce an error of order at most $O\left(e^{-2 \bar{\tau}}\right)$ that can be accounted for to give

$$
\begin{aligned}
R_{\sigma}= & R_{\eta \eta}+\left(2 H_{0}-1\right) R_{\eta}+2 H_{0}^{\prime} R+e^{-\tau} W^{\prime \prime}(\eta)+\left(2 H_{0}-1\right) e^{-\tau} W^{\prime}(\eta)+2 H_{0}^{\prime} e^{-\tau} W \\
& +e^{-\tau}\left[\left(2 H_{0}-1\right) H_{0}-\eta H_{0}^{\prime}+H_{0}^{\prime}\right]+O\left(C_{M} e^{-2 \bar{\tau}}\right) \\
= & R_{\eta \eta}+\left(2 H_{0}-1\right) R_{\eta}+2 H_{0}^{\prime} R+O\left(C_{M} e^{-2 \bar{\tau}}\right),
\end{aligned}
$$

where we have used the definition of $W$. Therefore $R$ satisfies the following problem (5.99)

$$
\begin{aligned}
& R_{\sigma}=R_{\eta \eta}+\left(2 H_{0}-1\right) R_{\eta}+2 H_{0}^{\prime} R+O\left(C_{M} e^{-2 \bar{\tau}}\right) \quad \text { for } \sigma \in[\bar{s}, s], \quad|\eta| \leq 1 / 2, \\
& R(\eta, \sigma)=O\left(C_{M} e^{-\bar{\tau}}\right) \quad \text { for } \sigma \in[\bar{s}, s], \quad|\eta| \leq 1 / 2, \\
& R(\eta, \bar{s})=O\left(\varepsilon e^{-\bar{\tau}}\right) \quad \text { for }|\eta| \leq 1 / 2
\end{aligned}
$$

Moreover, by parabolic regularity we also have

$$
\left|R_{\eta}(\eta, \bar{s})\right|+\left|R_{\eta \eta}(\eta, \bar{s})\right|+\left|R_{\sigma}(\eta, \bar{s})\right|=O\left(\varepsilon e^{-\bar{\tau}}\right) .
$$

on the same region. Let $\chi$ be a $C^{2}$ function of compact support such that $\chi(\eta)=0$ if $|\eta| \geq 1, \chi(\eta)=1$ if $|\eta| \leq 1 / 2$, and define

$$
\mathcal{R}(\eta, \sigma)=\chi(\eta) R(\eta, \sigma) .
$$

A routine calculation shows that $\mathcal{R}$ satisfies

$$
\begin{aligned}
\mathcal{R}_{\sigma}= & \mathcal{R}_{\eta \eta}+\left(2 H_{0}-1\right) \mathcal{R}_{\eta}+2 H_{0}^{\prime} \mathcal{R}-2 \chi^{\prime} R_{\eta}-\chi^{\prime \prime} \mathcal{R} \\
& -\left(2 H_{0}-1\right) \chi^{\prime} \mathcal{R}+\chi O\left(C_{M} e^{-2 \bar{\tau}}\right) .
\end{aligned}
$$

Since this equation is linear, we may estimate $\mathcal{R}$ as follows. Write $\mathcal{R}=\mathcal{R}_{1}+\mathcal{R}_{2}+\mathcal{R}_{3}$. Then the last term, $\mathcal{R}_{3}$, is such that

$$
\mathcal{R}_{3 \sigma}=O\left(C_{M} e^{-2 \bar{\tau}}\right), \quad \mathcal{R}_{3}(0)=0,
$$


and therefore it can be bounded in modulus by the solution $\bar{R}_{3}=K_{M}(\sigma-\bar{s}) e^{-2 \bar{\tau}}$ of

$$
\bar{R}_{3 \sigma}=K_{M}\left(M e^{-2 \bar{\tau}}\right), \quad \bar{R}_{3}(\bar{s})=0,
$$

with $K_{M}$ suficiently large. The second function, $\mathcal{R}_{2}$, is the solution of

$$
\begin{aligned}
& \mathcal{R}_{2 \sigma}=\mathcal{R}_{2 \eta \eta}+\left(2 H_{0}-1\right) \mathcal{R}_{2 \eta}+2 H_{0}^{\prime} \mathcal{R}_{2}-2 \mathcal{R}_{\eta} \chi^{\prime}-\mathcal{R} \chi^{\prime \prime}-\left(2 H_{0}-1\right) \mathcal{R} \chi^{\prime} \\
& \mathcal{R}_{2}(\eta, \bar{s})=0
\end{aligned}
$$

and therefore $\left|\mathcal{R}_{2}\right| \leq \bar{R}_{2}$, where $\bar{R}_{2}$ solves

$$
\begin{aligned}
& \bar{R}_{2 \sigma}=\bar{R}_{2 \eta \eta}+\left(2 H_{0}-1\right) \bar{R}_{2 \eta}+2 H_{0}^{\prime} \bar{R}_{2}+2\left|\mathcal{R}_{\eta} \chi^{\prime}\right|+\left|\mathcal{R} \chi^{\prime \prime}\right|+\left|\left(2 H_{0}-1\right) \mathcal{R} \chi^{\prime}\right| \\
& \bar{R}_{2}(\eta, \bar{s})=0 .
\end{aligned}
$$

This is a linear equation with constant coefficients, whose solution is given by

$$
\bar{R}_{2}(\eta, \sigma)=\int_{\bar{s}}^{\sigma} \int_{\mathbf{R}} G(\eta-\bar{\eta}, \rho-\bar{s}) F(\bar{\eta}, \rho) d \bar{\eta} d \rho,
$$

where we have set $F(\eta, \sigma)=2\left|\mathcal{R}_{\eta} \chi^{\prime}\right|+\left|\mathcal{R} \chi^{\prime \prime}\right|+\left|\left(2 H_{0}-1\right) \mathcal{R} \chi^{\prime}\right|$, and where $G$ is the Green function of the linear operator with nonconstant coefficients

$$
\bar{R}_{2 \sigma}=\bar{R}_{2 \eta \eta}+\left(2 H_{0}-1\right) \bar{R}_{2 \eta}+2 H_{0}^{\prime} \bar{R}_{2} .
$$

It is known (cf. [A]) that there exist positive constants $a$ and $\gamma$ such that

$$
G(\eta-\bar{\eta}, \rho-\bar{s}) \leq \gamma(\rho-\bar{s})^{-\frac{1}{2}} e^{-a \frac{|\eta-\pi|^{2}}{\rho-\bar{s}}} .
$$

Hence

$$
\left|\bar{R}_{2}(\eta, \sigma)\right| \leq \gamma \int_{\bar{s}}^{\sigma} \int_{\mathbf{R}}(\rho-\bar{s})^{-\frac{1}{2}} e^{-a \frac{|\eta-\bar{\eta}|^{2}}{\rho-\bar{s}}} F(\bar{\eta}, \rho) d \bar{\eta} d \rho .
$$

By construction, $F(\overline{\eta)}$ is zero if $|\bar{\eta}| \leq 1 / 2$ or $|\bar{\eta}| \geq 1$. Therefore, if $|\eta| \leq 1 / 4$ we deduce that

$$
\begin{aligned}
& \left|\bar{R}_{2}(\eta, \sigma)\right| \leq \gamma C_{M} e^{-\bar{\tau}} \int_{\bar{s}}^{\sigma} \int_{\frac{1}{2} \leq|\bar{\eta}| \leq 1}(\rho-\bar{s})^{-\frac{1}{2}} e^{-a \frac{|\bar{\eta}|^{2}}{4(\rho-\bar{s})}} d \bar{\eta} d \rho \\
& \leq \gamma C_{M} e^{-\bar{\tau}} \int_{\bar{s}}^{\sigma}(\rho-\bar{s})^{-\frac{1}{2}} e^{\frac{-a}{4(\rho-\bar{s})}} d \bar{\eta} \\
& \leq C_{M} e^{-\bar{\tau}}(\sigma-\bar{s})^{\frac{3}{2}} e^{-\frac{a}{4(\sigma-\bar{s})}} .
\end{aligned}
$$

This implies that, uniformly on $\sigma \in[\bar{s}, s]$ and $|\eta| \leq 1 / 4$,

$$
\left|\mathcal{R}_{2}\right| \leq\left|\bar{R}_{2}\right| \leq C_{M} \varepsilon e^{-\bar{\tau}},
$$

whence, by parabolic regularity, for $\sigma \in(\bar{s}, s)$ and $|\eta| \leq 1 / 4$,

$$
\left|\mathcal{R}_{2 \theta}\right|+\left|\mathcal{R}_{2 \theta \theta}\right| \leq C_{M} \varepsilon e^{-\bar{\tau}} .
$$

Moreover, since $\left|\mathcal{R}_{2}(\eta, \bar{s})\right|<\bar{R}_{2}(\eta, \bar{s})$, we also have

$$
\left|\mathcal{R}_{2 \sigma}(\eta, \bar{s})\right| \leq \lim _{\sigma \longrightarrow \bar{s}} \frac{\bar{R}_{2}(\eta, \sigma)}{\sigma-\bar{s}}=0 .
$$


The term $\mathcal{R}_{1}$ will be estimated in a slightly different way. Recall that $\mathcal{R}_{1}$ satisfies $\mathcal{R}_{1 \sigma}=\mathcal{R}_{1 \eta \eta}+\left(2 H_{0}-1-2 \chi^{\prime}\right) \mathcal{R}_{1 \eta}+\left(2 H_{0}^{\prime}\right) \mathcal{R}_{1}$ for all $\sigma \in[\bar{s}, s]$ and $|\eta| \leq 1 / 4$, $\left|\mathcal{R}_{1}(\eta, \bar{s})\right|+\left|\mathcal{R}_{1 \eta \eta}(\eta, \bar{s})\right|+\left|\mathcal{R}_{1 \eta}(\eta, \bar{s})\right| \leq C \varepsilon e^{-\bar{\tau}}$ for all $|\eta| \leq 1 / 4$, $\mathcal{R}_{1}\left( \pm \frac{1}{2}, \sigma\right)=O\left(C_{M} e^{-\bar{\tau}}\right)$, for $\sigma \in[\bar{s}, s]$.

Therefore, by parabolic regularity we have

$$
\left|\mathcal{R}_{1}\right|+\left|\mathcal{R}_{1 \eta}\right|+\left|\mathcal{R}_{1 \eta \eta}\right|+\left|\mathcal{R}_{1 \sigma}\right| \leq C_{M} \varepsilon e^{-\bar{\tau}}
$$

uniformly on $\sigma \in[\bar{s}, s]$ and $|\eta| \leq 1 / 4$. We deduce from (5.102)-(5.105) that

$$
|R|+\left|R_{\theta}\right|+\left|R_{\theta \theta}\right|+\left|R_{\sigma}\right| \leq C_{M} \varepsilon e^{-\bar{\tau}}
$$

uniformly for $\sigma \in[\bar{s}, s]$ and $|\eta| \leq 1 / 4$. Remember now that $\lambda(\tau)$ is the unique point such that $\phi(\xi, \tau)=1 / 2$ and $\phi_{\xi}(\xi, \tau)<0$. By classical regularity theory, the curve $\xi=\lambda(\tau)$ is well defined for $\tau-\bar{\tau}$ small. Therefore, for all $\sigma \in[\bar{s}, s]$, one has that $\psi((\lambda(\tau)-\lambda(\bar{\tau})) \sigma, \sigma)=1 / 2$ and, therefore,

$$
\begin{aligned}
& H_{c\left(\bar{s}, \eta_{0}(\bar{s}), 0\right.}\left((\lambda(\tau)-\lambda(\bar{\tau})) e^{\tau}-c(\bar{s})\left(e^{\tau}-e^{\bar{\tau}}\right)\right)+e^{-\tau} W\left((\lambda(\tau)-\lambda(\bar{\tau})) e^{\tau}\right) \\
& +R\left((\lambda(\tau)-\lambda(\bar{\tau})) e^{\tau}, e^{\tau}\right)=\frac{1}{2}
\end{aligned}
$$

On differentiating with respect to $\tau$, we obtain

$$
\begin{aligned}
& \left(\dot{\lambda}(\tau) e^{\tau}+(\lambda(\tau)-\lambda(\bar{\tau})) e^{\tau}-c(\bar{s}) e^{\tau}\right) H_{c(\bar{s}), \eta_{0}(\bar{s}), 0}^{\prime}\left((\lambda(\tau)-\lambda(\bar{\tau})) e^{\tau}-c(\bar{s})\left(e^{\tau}-e^{\bar{\tau}}\right)\right) \\
& -e^{-\tau} W\left((\lambda(\tau)-\lambda(\bar{\tau})) e^{\tau}\right)+e^{-\tau}\left(\dot{\lambda}(\tau) e^{\tau}+(\lambda(\tau)-\lambda(\bar{\tau})) e^{\tau}\right) W^{\prime}\left((\lambda(\tau)-\lambda(\bar{\tau})) e^{\tau}\right) \\
& +e^{\tau} R_{\sigma}\left((\lambda(\tau)-\lambda(\bar{\tau})) e^{\tau}, e^{\tau}\right)+\left(\dot{\lambda}(\tau) e^{\tau}+(\lambda(\tau)-\lambda(\bar{\tau})) e^{\tau}\right) R_{\theta}\left((\lambda(\tau)-\lambda(\bar{\tau})) e^{\tau}, e^{\tau}\right) \\
& =0 .
\end{aligned}
$$

Taking $\tau=\bar{\tau}$, we deduce that

$$
\begin{gathered}
\left(\dot{\lambda}(\tau) e^{\tau}-c(s) e^{\tau}\right) H_{c(s), \eta_{0}(s), 0}^{\prime}(0)-e^{-\tau} W(0)+\dot{\lambda}(\tau) W^{\prime}(0) \\
+e^{\tau} R_{\sigma}\left(0, e^{\tau}\right)+\dot{\lambda}(\tau) e^{\tau} R_{\theta}\left(0, e^{\tau}\right)=0
\end{gathered}
$$

whence

$$
\begin{aligned}
\dot{\lambda}(\tau) & =\frac{c(s)}{\left(1+R_{\eta}\left(0, e^{\tau}\right)\right)}+\frac{e^{-\tau} W(0)-\dot{\lambda}(\tau) W^{\prime}(0)-e^{\tau} R_{\sigma}\left(0, e^{\tau}\right)}{e^{\tau}\left(1+R_{\eta}\left(0, e^{\tau}\right)\right) H_{c(s), \eta_{0}(s), 0}^{\prime}(0)} \\
& =c(s)+C_{1} e^{-\tau}+O\left(\varepsilon e^{-\tau}\right)=\lambda(\tau)+C_{1} e^{-\tau}+O\left(\varepsilon e^{-\tau}\right) .
\end{aligned}
$$

As a consequence of the previous lemma we have

Corollary 5.14. Assume that $\phi \in \mathcal{A}\left(M ; \tau_{0}, \tau_{1}\right)$ is a solution of (4.5) with initial data $\phi\left(\xi, \tau_{0}\right)$ given as before. Suppose that

$$
l_{1}\left(\alpha_{0}, k_{0}, \tau_{1}\right) \equiv\left\langle L_{0}, \psi\left(\lambda, \tau_{1}\right)\right\rangle=0,
$$

where $\left\langle L_{0}, \psi\left(\lambda, \tau_{1}\right)\right\rangle$ is defined in (5.14) and $k_{0}=\lambda\left(\tau_{0}\right)$, and let $C_{1}$ and $g$ be given as in the previous lemma. Then, if

$$
l_{2}\left(\alpha_{0}, k_{0} ; \tau_{1}\right) \equiv k_{0}+e^{-\tau_{0}} \int_{\tau_{0}}^{\tau_{1}} e^{-2 \sigma}\left(C_{1}+\varepsilon g(\sigma)\right) d \sigma=0
$$


there exists a positive constant $C$, independent of $M$, such that for all $\tau \in\left[\tau_{0}, \tau_{1}\right]$

$$
|\lambda(\tau)| \leq C e^{-\tau}
$$

Proof. Let us integrate (5.96) between $\tau_{0}$ and $\tau$, to obtain

$$
\lambda(\tau)=\lambda\left(\tau_{0}\right) e^{\tau-\tau_{0}}+e^{\tau} \int_{\tau_{0}}^{\tau} e^{-2 \sigma}\left(C_{1}+\varepsilon g(\sigma)\right) d \sigma .
$$

Suppose now that $\lambda\left(\tau_{0}\right)$ is such that

$$
\lambda\left(\tau_{0}\right) e^{-\tau_{0}}+\int_{\tau_{0}}^{\tau_{1}} e^{-2 \sigma}\left(C_{1}+\varepsilon g(\sigma)\right) d \sigma=0
$$

or equivalently

$$
\lambda\left(\tau_{0}\right)=-e^{\tau_{0}} \int_{\tau_{0}}^{\tau_{1}} e^{-2 \sigma}\left(C_{1}+\varepsilon g(\sigma)\right) d \sigma
$$

We have then that for every $\tau \in\left[\tau_{0}, \tau_{1}\right]$

$$
\begin{aligned}
& \lambda(\tau)=e^{\tau}\left(\int_{\tau_{0}}^{\tau} e^{-2 \sigma}\left(C_{1}+\varepsilon g(\sigma)\right) d \sigma-\int_{\tau_{0}}^{\tau_{1}} e^{-2 \sigma}\left(C_{1}+\varepsilon g(\sigma)\right) d \sigma\right) \\
& =\int_{\tau}^{\tau_{1}} e^{-2 \sigma}\left(C_{1}+\varepsilon g(\sigma)\right) d \sigma \leq \frac{C_{1}+\varepsilon g(\sigma)}{2} e^{-\tau}
\end{aligned}
$$

and the lemma follows.

Remark. Observe that if $k_{0}$ is such that (5.106) holds, then, for $M$ large enough, the condition (5.1) of the definition of $\mathcal{A}\left(M ; \tau_{0}, \tau_{1}\right)$ is fulfilled in all the interval $\left[\tau_{0}, \tau_{1}\right]$ with the constant $M$ replaced (for instance) by $M / 2$.

As a consequence of Lemmata 5.1 to 5.13 and Corollary 5.14, we have the following

Proposition 5.15. Assume that $M>0$ is sufficiently large and $\varepsilon$ is small enough. Let $\phi$ be a solution of (4.5) with initial data $\phi\left(\xi, \tau_{0}\right)$ given as before and such that

$$
\phi(\xi, \tau) \in \overline{\mathcal{A}\left(M ; \tau_{0}, \tau_{1}\right)}
$$

for $\tau \in\left[\tau_{0}, \tau_{1}\right]$ with $\tau_{1}>\tau_{0} \gg 1$. If

$$
l_{1}\left(\alpha_{0}, k_{0}\right)=\left\langle L_{0}, \psi\left(\lambda, \tau_{1}\right)\right\rangle=0
$$

and

$$
l_{2}\left(\alpha_{0}, k_{0}\right)=k_{0}+e^{-\tau_{0}} \int_{\tau_{0}}^{\tau_{1}} e^{-2 \sigma}\left(C_{1}+\varepsilon g(\sigma)\right) d \sigma=0
$$

then

$$
\phi(\xi, \tau) \in \mathcal{A}\left(M / 2 ; \tau_{0}, \tau_{1}\right)
$$


5.4. Existence of singularity-forming solutions the topological argument. For every given $\tau_{0}$ and $\tau_{1}$ with $\tau_{0} \leq \tau_{1}$, let us consider the subset $\mathcal{U}\left(\tau_{0}, \tau_{1}\right)$ of $\mathbf{R}^{2}$ consisting of the pairs $\left(\alpha_{0}, k_{0}\right)$ for which the solution of equation (4.5) with initial data determined by $\left(\alpha_{0}, k_{0}\right)$ belongs to $\mathcal{A}\left(M ; \tau_{0}, \tau_{1}\right)$. We shall denote this solution by $\phi\left(\xi, \tau ; \alpha_{0}, k_{0}\right)$. By classical continuous dependence results for parabolic equations, the set $\mathcal{U}\left(\tau_{0}, \tau_{1}\right)$ is an open subset of $\mathbf{R}^{2}$. Let $\ell \mathbf{R}^{3} \longrightarrow \mathbf{R}^{2}$ be the mapping defined by

$$
\ell(\alpha, k ; \tau)=\left(l_{1}(\alpha, k ; \tau), l_{2}(\alpha, k ; \tau)\right) .
$$

By our choice of the initial data the application $\ell$ is $C^{\infty}$ with respect to $\alpha, k$ and $\tau$. We next denote by $d\left(\ell, \mathcal{U}\left(\tau_{0}, \tau\right) ; 0\right)$ the topological degree of the mapping $\ell(\cdot ; \tau)$ in the set $\mathcal{U}\left(\tau_{0}, \tau\right)$ at the value 0 . It is clear that there exists a unique point $\left(\alpha_{0}, k_{0}\right) \in \mathcal{U}\left(\tau_{0}, \tau_{0}\right)$ such that $\ell\left(\alpha_{0}, k_{0}, \tau_{0}\right)=0$, which is $\alpha_{0}=k_{0}=0$. Therefore,

$$
d\left(\ell, \mathcal{U}\left(\tau_{0}, \tau_{0}\right) ; 0\right)=1 \text {. }
$$

Moreover,

$$
\begin{array}{ll}
\frac{\partial l_{1}}{\partial \alpha}\left(0,0 ; \tau_{0}\right)>0, & \frac{\partial l_{1}}{\partial k}\left(0,0 ; \tau_{0}\right)=0, \\
\frac{\partial l_{2}}{\partial \alpha}\left(0,0 ; \tau_{0}\right)=0, & \frac{\partial l_{2}}{\partial k}\left(0,0 ; \tau_{0}\right)=1 .
\end{array}
$$

Then, by the implicit function theorem, there is a small neighbourough of $\left(0,0, \tau_{0}\right)$ in which the set of points where the function $\ell$ is zero is given by a parametrised continuous curve $\{(\alpha(\rho), k(\rho), \rho)\}_{\rho \in\left[\tau_{0}, \rho_{0}\right]}$. On the other hand, by the above mentioned continuous dependence, if $\tau_{1}-\tau_{0}$ is sufficiently small the solutions $\phi(\xi, \tau, \alpha(\rho), k(\rho))$ (with initial data determined by $(\alpha(\rho), k(\rho)))$ belong to $\mathcal{A}\left(M ; \tau_{0}, \tau_{1}\right)$. This implies that if $\tau_{1}-\tau_{0}$ is sufficiently small, then

$$
d\left(\ell, \mathcal{U}\left(\tau_{0}, \tau_{1}\right) ; 0\right)=1 \text {. }
$$

We now claim that (5.109) holds for all $\tau>\tau_{0}$, provided that

$$
\mathcal{U}\left(\tau_{0}, \tau\right) \neq \emptyset \text {. }
$$

Suppose on the contrary that there exists a first time $\tau_{2}>\tau_{0}$ when (5.109) fails but (5.110) holds true. Then there must be a point

$$
(\alpha, k) \in \partial \mathcal{U}\left(\tau_{0}, \tau_{2}\right)
$$

where $\ell\left(\alpha, k ; \tau_{2}\right)=0$. Since $(\alpha, k) \in \partial \mathcal{U}\left(\tau_{0}, \tau_{2}\right)$, the solution $\phi(\xi, \tau ; \alpha, k)$ belongs to $\overline{\mathcal{A}\left(M ; \tau_{0}, \tau_{2}\right)}$. But then, using Proposition 5.15, we deduce that $\phi \in \mathcal{A}\left(M / 2 ; \tau_{0}, \tau_{2}\right)$ and therefore that $(\alpha, k) \in \mathcal{U}\left(\tau_{0}, \tau_{2}\right)$, which is a contradiction.

We further observe that, if $\tau_{0}$ is sufficiently large,

$$
\mathcal{U}\left(\tau_{0}, \tau\right) \neq \emptyset \text { for any } \tau>\tau_{0} .
$$

In order to check this, we define

$$
\tau^{*}=\sup \left\{\tau ; \mathcal{U}\left(\tau_{0}, \tau\right) \neq \emptyset\right\} .
$$

We already know that $\tau^{*}>\tau_{0}$. Assume now that $\tau^{*}<\infty$. By (5.109) and (5.110) we may select a sequence of times $\left\{\tau_{n}\right\}$ increasing to $\tau^{*}$, and a sequence $\left\{\left(\alpha_{n}, k_{n}\right)\right\}$ such that $\ell\left(\alpha_{n}, k_{n} ; \tau_{n}\right)=0$ and $\left(\alpha_{n}, k_{n}\right) \in \mathcal{U}\left(\tau_{0}, \tau_{n}\right)$. Since $\mathcal{U}\left(\tau_{0}, \tau_{n+1}\right) \subset \mathcal{U}\left(\tau_{0}, \tau_{n}\right)$, the sequence $\left\{\left(\alpha_{n}, k_{n}\right)\right\}$ is bounded. Therefore a subsequence, still denoted by $\left\{\left(\alpha_{n}, k_{n}\right)\right\}$, exists which converges to some point $\left(\alpha^{*}, k^{*}\right)$. By continuity of $\ell$ we still have $\ell\left(\alpha^{*}, k^{*} ; \tau^{*}\right)=0$. By Proposition 5.15 , the solution $\phi\left(\xi, \tau, \alpha^{*}, k^{*}\right)$ belongs 
to $\mathcal{A}\left(M ; \tau_{0}, \tau^{*}\right)$ and so, by continuous dependence, belongs to $\mathcal{A}\left(M ; \tau_{0}, \tau^{*}+\delta\right)$ for some $\delta>0$ sufficiently small. But this contradicts the definition of $\tau^{*}$.

We may now detail the argument leading to the existence of the solutions referred to in Theorem 3. We take a sequence $\left\{\tau_{n}\right\}$ such that $\tau_{1}>\tau_{0}$ and $\tau_{n} \longrightarrow \infty$ as $n \longrightarrow \infty$. For every $n$, as we have seen, $\mathcal{U}\left(\tau_{0}, \tau_{n}\right) \neq \emptyset$ and

$$
d\left(\ell, \mathcal{U}\left(\tau_{0}, \tau_{n}\right) ; 0\right)=1 .
$$

Let $\left(\alpha_{n}, k_{n}\right) \in \mathcal{U}\left(\tau_{0}, \tau_{n}\right)$ be such that $\ell\left(\alpha_{n}, k_{n} ; \tau_{n}\right)=0$, and denote by $\phi_{n}$ the solution $\phi\left(\xi, \tau, \alpha_{n}, k_{n}\right)$. By Proposition 5.15 we have

$$
\phi_{n} \in \mathcal{A}\left(M / 2 \tau_{0}, \tau_{n}\right) \text { for all } n \geq 1 \text {. }
$$

Since on the other hand the sequence $\left\{\left(\alpha_{n}, k_{n}\right)\right\}$ is bounded, there exist a subsequence, still denoted by $\left\{\left(\alpha_{n}, k_{n}\right)\right\}$, and a pair $\left(\bar{\alpha}_{0}, \bar{k}_{0}\right)$ such that

$$
\alpha_{n} \longrightarrow \bar{\alpha}_{0}, \quad k_{n} \longrightarrow \bar{k}_{0} .
$$

Let us consider the solution $\phi\left(\xi, \tau ; \bar{\alpha}_{0}, \bar{k}_{0}\right)$. By continuous dependence on the initial data we have, for fixed $m \geq 1$,

$$
\lim _{n \longrightarrow \infty} \phi_{n}(\xi, \tau)=\phi\left(\xi, \tau ; \bar{\alpha}_{0}, \bar{k}_{0}\right)
$$

uniformly for $\xi \in \mathbf{R}$ and $\tau \in\left[\tau_{0}, \tau_{m}\right]$. By (5.111), for every $n \geq m, \phi_{n} \in$ $\mathcal{A}\left(M / 2 ; \tau_{0}, \tau_{m}\right)$; and then, passing to the limit as $n \longrightarrow \infty$, we obtain $\phi\left(\xi, \tau ; \bar{\alpha}_{0}, \bar{k}_{0}\right)$ $\in \overline{\mathcal{A}\left(M / 2 ; \tau_{0}, \tau_{m}\right)} \subset \mathcal{A}\left(M ; \tau_{0}, \tau_{m}\right)$ for every $m \geq 1$. This shows that $\phi\left(\xi, \tau ; \bar{\alpha}_{0}, \bar{k}_{0}\right)$ is actually the desired solution of (4.5). In particular, since $\phi\left(\xi, \tau ; \bar{\alpha}_{0}, \bar{k}_{0}\right)$ belongs to $\mathcal{A}\left(M / 2 ; \tau_{0}, \tau\right)$ for all $\tau \geq \tau_{0}$, we deduce that Lemma 5.2 and Lemma 5.3 hold for $\tau \geq \tau_{0}$. Writing (5.28) and (5.35) in terms of the original variables then gives (1.13).

5.5. Analysis of the external region. We deal now with the question of obtaining the asymptotic estimate (1.14) for the global solution constructed in the previous sections. To this end we observe that, in terms of the variables $\xi, \tau$ and $\phi$ introduced in (4.4), it is sufficient to obtain

$$
\left|\phi(\xi, \tau)-e^{-\xi}\right| \leq \varepsilon\left(\tau_{1}\right) e^{-\xi},
$$

uniformly on $\tau \geq \tau_{1}, \xi \in\left(x_{1}, \tau-\tau_{1}+2 x_{1}\right)$, where $\lim _{\tau_{1} \longrightarrow \infty} \varepsilon\left(\tau_{1}\right)=0$, since in the original variables this means $\left|x^{2} f(x, t)-1\right| \leq \varepsilon\left(\tau_{1}\right)$ uniformly for $t \in\left(1-e^{-\tau_{1}}, 1\right)$ and $x \in\left(2(1-t), e^{2 x_{1}-\tau_{1}}\right)$. From the estimates obtained in Subsections 5.1-5.4, we easily deduce that for all $\tau_{1} \geq \tau_{0}$ and all positive constant $L$ there is an $\varepsilon\left(\tau_{1}\right)$ such that

$$
\left|\phi(\xi, \tau)-e^{-\xi}\left(1+2 e^{-\tau}\right)\right| \leq \varepsilon\left(\tau_{1}\right) e^{-\tau} \quad \text { for all } \xi \in\left(x_{1}, L\right) \quad \text { and } \tau \geq \tau_{1},
$$

with $\varepsilon\left(\tau_{1}\right) \longrightarrow 0$ as $\tau_{1} \longrightarrow \infty$. Therefore

$$
\left|\phi(\xi, \tau)-e^{-\xi}\right| \leq \varepsilon\left(\tau_{1}\right) e^{-\xi} \text { for all } \xi \in\left(x_{1}, L\right) \text { and } \tau \geq \tau_{1} .
$$

We need to extend this estimate to a larger region containing $\xi \in\left(x_{1}, \tau-\tau_{1}+2 x_{1}\right)$. To this end, let us introduce the function $W$ given by

$$
\phi(\xi, \tau)=\bar{\phi}(\xi)+e^{-\tau} \phi_{1}(\xi)+W(\xi, \tau) .
$$


A straightforward computation shows that $W$ satisfies

$$
\begin{aligned}
W_{\tau}= & e^{-\tau}\left(W_{\xi \xi}+W_{\xi}-2 W\right)+(2 \bar{\phi}-1)\left(W+W_{\xi}\right)+2 W\left(W+W_{\xi}\right) \\
& +2 \phi_{1} e^{-\tau}\left(W+W_{\xi}\right)+e^{-2 \tau+\xi}\left(3 W+W_{\xi}\right)+e^{-2 \tau+\xi}\left(2 \bar{\phi}+2 e^{-\tau} \phi_{1}\right) \\
& +e^{-2 \tau}\left(\phi_{1 \xi \xi}+\phi_{1 \xi}-2 \phi_{1}\right) .
\end{aligned}
$$

By (5.112) we easily check that, for $\tau \geq \tau_{1}$ and $\xi \in\left(x_{1}, L\right)$,

$$
|W(\xi, \tau)| \leq \varepsilon\left(\tau_{1}\right) e^{-\tau} .
$$

On the other hand, since the solution $\phi$ belongs to $\mathcal{A}\left(M ; \tau_{0}, \tau\right)$ for every $\tau \geq \tau_{0}$, one has

$$
|W(\xi, \tau)| \leq M e^{-\tau} \text { for all } \xi \in \mathbf{R} \text { and } \tau \geq \tau_{1} .
$$

In order to obtain a better estimate of $W$, let us write

$$
W(\xi, \tau)=\varepsilon e^{-\tau}+\varphi+G,
$$

where the function $\varphi$ is the solution of

$$
\left\{\begin{array}{l}
\varphi_{\tau}=e^{-\tau}-\left(\varphi+\varphi_{\xi}\right) \text { for } \xi>0 \text { and } \tau \geq \tau_{1} \\
\phi\left(\xi, \tau_{1}\right)=\varphi_{0}(\xi) \text { for } \xi>0
\end{array}\right.
$$

and $\varphi_{0}$ is a nonnegative, monotone increasing smooth function such that

$$
\varphi_{0}(\xi)+\varepsilon\left(\tau_{1}\right) e^{-\tau_{1}} \geq W\left(\xi, \tau_{1}\right), \quad \text { for } \xi \in \mathbf{R} .
$$

Due to (5.117), we may choose $\varphi_{0}$ in the following form

$$
\varphi_{0}(\xi)=\left\{\begin{array}{l}
0 \quad \text { if } \xi<\zeta_{0}, \\
M e^{-\tau_{1}} \quad \text { if } \xi>2 \zeta_{0},
\end{array}\right.
$$

with $\zeta_{0}>2 x_{1}$ being a sufficiently large fixed value. Setting

$$
\begin{aligned}
& \varphi(\xi, \tau)=e^{-\left(\tau-\tau_{1}\right)} z\left(\left(\xi-\zeta_{0}\right)-\left(\tau-\tau_{1}\right), s\right)=e^{-\left(\tau-\tau_{1}\right)} z(\theta, s) \\
& \text { with } s=e^{-\tau_{1}}-e^{-\tau}, \quad \theta=\left(\xi-\zeta_{0}\right)-\left(\tau-\tau_{1}\right),
\end{aligned}
$$

we readily see that

$$
z_{s}=z_{\theta \theta}
$$

and therefore

$$
\varphi(\xi, \tau)=\frac{1}{2 \sqrt{\pi}} e^{-\left(\tau-\tau_{1}\right)} \int_{-\infty}^{\frac{\left(\xi-\zeta_{0}\right)-\left(\tau-\tau_{1}\right)}{2 \sqrt{e^{-\tau}-e^{-\tau}}}} e^{-r^{2}} \varphi_{0}\left(\xi-\tau+\tau_{1}-2 r \sqrt{e^{-\tau_{1}}-e^{-\tau}}\right) d r .
$$

On the other hand, $G$ satisfies

$$
\begin{aligned}
G_{\tau}= & e^{-\tau}\left(G_{\xi \xi}+G_{\xi}-2 G\right)+(2 \bar{\phi}-1)\left(G+G_{\xi}\right)+2 \phi_{1} e^{-\tau}\left(G+G_{\xi}\right) \\
& +e^{-2 \tau+\xi}\left(3 G+G_{\xi}\right)+2 G\left(G+G_{\xi}\right)+2\left(\varepsilon e^{-\tau}+\varphi\right)\left(G+G_{\xi}\right) \\
+ & G\left(\varepsilon e^{-\tau}+\varphi+\varphi_{\xi}\right)+2 \bar{\phi}\left(\varepsilon e^{-\tau}+\varphi+\varphi_{\xi}\right)+J_{1}(\xi, \tau),
\end{aligned}
$$

with

$$
\begin{aligned}
J_{1}(\xi, \tau) & =e^{-\tau}\left(\varphi_{\xi}-2 \varphi\right)+2\left(\varepsilon e^{-\tau}+\varphi\right)\left(\varepsilon e^{-\tau}+\varphi+\varphi_{\xi}\right)-2 \varepsilon e^{-2 \tau} \\
& +2 \phi_{1} e^{-\tau}\left(\varepsilon e^{-\tau}+\varphi+\varphi_{\xi}\right)+e^{-2 \tau+\xi}\left(3 \varepsilon e^{-\tau}+3 \varphi+\varphi_{\xi}\right) \\
& +e^{-2 \tau+\xi}\left(2 \bar{\phi}+2 e^{-\tau} \phi_{1}\right)+e^{-2 \tau}\left(\phi_{1 \xi \xi}+\phi_{1 \xi}-2 \phi_{1}\right) .
\end{aligned}
$$


By the estimates that we have already obtained, there exists a positive constant $C_{M}$, depending on $M$, such that, for every $\tau \geq \tau_{0}$ and all $\xi \in \mathbf{R}$,

$$
\left|J_{1}(\xi, \tau)\right|+\left|\frac{\partial J_{1}}{\partial \xi}(\xi, \tau)\right|+\left|\frac{\partial^{2} J_{1}}{\partial \xi^{2}}(\xi, \tau)\right| \leq C_{M} e^{-2 \tau} .
$$

On the other hand, using the explicit expression of $\varphi$, we see that there exists a positive constant $C_{M}$ such that for all $\tau$ and $\xi \in \mathbf{R}$

$$
\left|\bar{\phi}(\xi)\left(\varphi(\xi, \tau)+\varphi_{\xi}(\xi, \tau)\right)\right| \leq C_{M} e^{-2 \tau} .
$$

Finally, let us define the function $H$ by

$$
G(\xi, \tau)=H(\xi, \tau)-2 \varepsilon \phi_{1}(\xi) e^{-\tau},
$$

so that $H$ satisfies

$$
\left\{\begin{aligned}
H_{\tau} & =e^{-\tau}\left(H_{\xi \xi}+H_{\xi}-2 H\right)+(2 \bar{\phi}-1)\left(H+H_{\xi}\right)-2 \phi_{1} e^{-\tau}\left(H+H_{\xi}\right) \\
& +e^{-2 \tau+\xi}\left(3 H+H_{\xi}\right)+\left(2 \varepsilon e^{-\tau}+2 \varphi-4 \varepsilon \phi_{1} e^{-\tau}+2 H\right)\left(H+H_{\xi}\right) \\
& +H\left(-4 \varepsilon e^{-\tau} \phi_{1}-4 \varepsilon \phi_{1}^{\prime} e^{-\tau}+\varepsilon e^{-\tau}+\varphi+\varphi_{\xi}\right) \\
& +2 \bar{\phi}\left(\varphi+\varphi_{\xi}\right)+J_{1}(\xi, \tau)+J_{2}(\xi, \tau)
\end{aligned}\right.
$$

where the term $J_{2}$ is now given by

$$
\begin{aligned}
& J_{2}(\xi, \tau)=e^{-\tau}\left(2 \varepsilon \phi_{1}^{\prime \prime} e^{-\tau}-2 \varepsilon \phi_{1}^{\prime} e^{-\tau}+4 \varepsilon \phi_{1} e^{-\tau}\right)-2 \phi_{1} e^{-\tau}\left(2 \varepsilon \phi_{1} e^{-\tau}+2 \varepsilon \phi_{1}^{\prime} e^{-\tau}\right) \\
& -e^{-2 \tau+\xi}\left(6 \varepsilon \phi_{1} e^{-\tau}+2 \varepsilon \phi_{1}^{\prime} e^{-\tau}\right)+\left(2 \varepsilon e^{-\tau}+2 \varphi-4 \varepsilon \phi_{1} e^{-\tau}\right)\left(2 \varepsilon e^{-\tau} \phi_{1}+2 \varepsilon \phi_{1}^{\prime} e^{-\tau}\right) \\
& +2 \varepsilon e^{-\tau} \phi_{1}\left(\varepsilon e^{-\tau}+\varphi+\varphi_{\xi}\right)
\end{aligned}
$$

and satisfies an estimate similar to $(5.124)$ for some positive constant $C_{M}$. One readily sees that

$$
\left\{\begin{array}{l}
H\left(x_{1}, \tau\right) \leq 2 \varepsilon\left\|\phi_{1}\right\|_{\infty} e^{-\tau}, \quad \text { for } \tau \geq \tau_{1}, \\
H\left(\tau-\tau_{1}+2 x_{1}, \tau\right) \leq 2 \varepsilon\left\|\phi_{1}\right\|_{\infty} e^{-\tau}, \quad \text { for } \tau \geq \tau_{1}, \\
H\left(\xi, \tau_{1}\right) \leq 2 \varepsilon\left\|\phi_{1}\right\|_{\infty} e^{-\tau_{1}} \quad \text { for } x_{1}<\xi<\tau-\tau_{1}+2 x_{1} .
\end{array}\right.
$$

Consider now the solution $\bar{H}$ of the following linear hyperbolic problem

$$
\begin{aligned}
& \bar{H}_{\tau}=(2 \bar{\phi}-1)\left(\bar{H}+\bar{H}_{\xi}\right)+K_{M} e^{-2 \tau} \quad \text { for } x_{1}<\xi<\tau-\tau_{1}+2 x_{1}, \tau \geq \tau_{0}, \\
& \bar{H}\left(\xi, \tau_{1}\right)=2\left\|\phi_{1}\right\|_{\infty} \varepsilon e^{-\tau_{1}} \quad \text { for } x_{1}<\xi<\tau-\tau_{1}+2 x_{1}, \\
& \bar{H}\left(x_{1}, \tau\right)=\bar{H}\left(\tau-\tau_{1}+2 x_{1}, \tau\right)=2 \varepsilon\left\|\phi_{1}\right\|_{\infty} e^{-\tau} \text { for } \tau \geq \tau_{1},
\end{aligned}
$$

where $K_{M}$ is taken such that

$$
\left(J_{1}(\xi, \tau)+J_{2}(\xi, \tau)\right) e^{2 \tau}<K_{M}, \quad \text { for } \tau \geq \tau_{0} .
$$

Notice that such a choice is possible since $J_{1}$ and $J_{2}$ satisfy (5.124). We first claim that there exists a positive constant $\bar{\varepsilon}\left(\tau_{1}\right)$ such that, for every $\tau \geq \tau_{1}$ and $\xi \in\left(x_{1}, \tau-\tau_{1}+2 x_{1}\right)$, we have

$$
|\bar{H}(\xi, \tau)| \leq \bar{\varepsilon}\left(\tau_{1}\right) e^{-\tau} .
$$

This follows by integrating the equation along characteristics. Moreover, differentiating in the equation satisfied by $H$ and using again the estimate (5.124) for $J_{1}$ and $J_{2}$, we deduce that there exists $\delta\left(\tau_{1}\right)>0$ such that

$$
\left|\bar{H}_{\xi}(\xi, \tau)\right|+\left|\bar{H}_{\xi \xi}(\xi, \tau)\right| \leq \delta\left(\tau_{1}\right) e^{-\tau}
$$


for all $\tau \geq \tau_{1}$ and $\xi \in\left(x_{1}, \tau-\tau_{1}+2 x_{1}\right)$, with $\delta\left(\tau_{1}\right) \longrightarrow 0$ as $\tau_{1} \longrightarrow \infty$. It then turns out that $\bar{H}$ is a supersolution of (5.20) and thus, by a standard comparison argument, for every $\tau \geq \tau_{1}$ and $\xi \in\left(x_{1}, \tau-\tau_{1}+2 x_{1}\right)$ we have

$$
H \leq \bar{\varepsilon}\left(\tau_{1}\right) e^{-\tau} \text {. }
$$

One can obtain a lower estimate in a similar way by using a suitable subsolution. We deduce from this argument that

$$
|H(\xi, \tau)| \leq \bar{\varepsilon}\left(\tau_{1}\right) e^{-\tau}
$$

for every $\tau \geq \tau_{1}$ and $\xi \in\left(x_{1}, \tau-\tau_{1}+2 x_{1}\right)$. This in turn implies that

$$
|W(\xi, \tau)| \leq \varepsilon e^{-\tau}+\varphi+\bar{\varepsilon}\left(\tau_{1}\right) e^{-\tau}
$$

in the same region. Now, for $\tau \geq 2 \tau_{1}$ and $\xi \in\left(x_{1}, \tau-\tau_{1}+2 x_{1}\right)$, we have

$$
\begin{aligned}
& \varphi(\xi, \tau) \leq \frac{e^{-\left(\tau-\tau_{1}\right)}}{2 \sqrt{\pi}} \int_{-\infty}^{\frac{2 x_{1}-\zeta_{0}}{2 \sqrt{e^{-\tau_{1}}-e^{-2 \tau_{1}}}}} e^{-r^{2}} \varphi_{0}\left(2 x_{1}-2 r \sqrt{e^{-\tau_{1}}-e^{-2 \tau_{1}}}\right) d r \\
& \leq e^{-\tau}\left\{\frac{K_{M}}{2 \sqrt{\pi}} \int_{-\infty}^{\frac{2 x_{1}-\zeta_{0}}{2 \sqrt{e^{-\tau_{1}}-e^{-2 \tau_{1}}}}} e^{-r^{2}} d r\right\} \\
& \sim e^{-\tau}\left\{\frac{K_{M}}{2 \sqrt{\pi}} \frac{\sqrt{e^{-\tau_{1}}-e^{-2 \tau_{1}}}}{\zeta_{0}-2 x_{1}} e^{-\frac{\left(\zeta_{0}-2 x_{1}\right)^{2}}{4\left(e^{\left.-\tau_{1}-e^{-2 \tau_{1}}\right)}\right.}}\right\}
\end{aligned}
$$

as $\tau_{1} \longrightarrow+\infty$ and whenever $\zeta_{0}>2 x_{1}$. Observe that if we set

$$
\gamma\left(\tau_{1}, M\right)=\frac{K_{M}}{2 \sqrt{\pi}} \frac{\sqrt{e^{-\tau_{1}}-e^{-2 \tau_{1}}}}{\zeta_{0}-2 x_{1}} e^{-\frac{\left(\zeta_{0}-2 x_{1}\right)^{2}}{4\left(e^{-\tau_{1}}-e^{-2 \tau_{1}}\right)}},
$$

we have, for all fixed $M$,

$$
\lim _{\tau_{1} \longrightarrow \infty} \gamma\left(\tau_{1}, M\right)=0
$$

By substituting this in (5.109) we obtain

$$
\begin{aligned}
\left|\Phi(\xi, \tau)-e^{-\xi}\right| & \leq e^{-\tau} \Phi_{1}(\xi)+\gamma\left(\tau_{1}, M\right) e^{-\tau} \\
& \leq e^{-\xi}\left(\left\|\Phi_{1}\right\|_{\infty}+\gamma\left(\tau_{1}, M\right)\right) e^{\xi-\tau} \\
& \leq e^{-\xi}\left(\left\|\Phi_{1}\right\|_{\infty}+\gamma\left(\tau_{1}, M\right)\right) e^{-\tau_{1}+2 x_{1}}
\end{aligned}
$$

whereupon the result follows with

$$
\varepsilon\left(\tau_{1}\right)=\left(\left\|\Phi_{1}\right\|_{\infty}+\gamma\left(\tau_{1}, M\right)\right) e^{-\tau_{1}+2 x_{1}} .
$$

\section{REFERENCES}

[AE] J. Aguirre and M. Escobedo, On the blow-up of solutions of a convective reaction-diffusion equation, Proc. Royal Soc. Edinburgh 123A, (1993), pp. 433-460. MR 94d:35076

[A] D. G. Aronson, Non-negative solutions of linear parabolic equations, Ann. Scuola Normale Sup. Pisa (3) 22 (1968), pp. 607-694. MR 55:8553, MR 55:8554

[CL] R. E. Caflisch and C. D. Levermore, Equilibrium for radiation in a homogeneous plasma, Phys. Fluids 29 (1986), pp. 748-752. MR 86c:76140 .

[HV1] M. A. Herrero and J. J. L. Velazquez, Blow-up behaviour of one-dimensional semilinear parabolic problems, Ann. Inst. Henri Poincaré, 10 (1993), pp. 131-189. MR 94g:35030

[HV2] M. A. Herrero and J. J. L. Velazquez, Generic behaviour of one-dimensional blow-up patterns, Ann. Scuola Normale Sup. Pisa (4) 19 (1992), pp. 381-450. MR 94b:35048

[HV3] M. A. Herrero and J. J. L. Velazquez, On the melting of ice balls, SIAM J. Math. Analysis 28 (1997), 1-32. MR 97m:35287

[K] A. S. Kompaneets, The establishment of thermal equilibrium between quanta and electrons, Soviet Physics JETP, 4, (1957), pp. 730-737. 
[KL] O. Kavian and C. D. Levermore, On the Kompaneets Equation, a singular semi-linear parabolic equation with blow-up. In preparation.

[NT] R. Natalini and A. Tesei, Blow-up of solutions for a class of balance laws, Comm. Part. Diff. Eq., 19 (1994), pp. 417-453. MR 95a:35087

[V1] J. J. L. Velazquez, Classification of singularities for blowing up solutions in higher dimensions, Trans. Amer. Math. Soc. 338 (1993), pp. 441-464. MR 93j:35101

[V2] J. J. L. Velazquez, Curvature blow-up in perturbations of minimal cones evolving by mean curvature flow, Ann. Scuola Normale Sup. Pisa (4) 21 (1994), pp. 595-628. MR 96f:53018

(M. Escobedo) Departamento de Matemáticas, Universidad del País Vasco, Apartado 644, 48080 Bilbao, Spain

E-mail address: mtpesmam@lg.ehu.es

(M. A. Herrero and J. J. L. Velazquez) Departamento de Matemática Aplicada, Facultad de Matemáticas, Universidad Complutense, 28040 Madrid, Spain

E-mail address: herrero@sunma4.mat.ucm.es

E-mail address: velazque@sunma4.mat.ucm.es 